\title{
ANALYSES OF DEFORMATION IN VISCOELASTIC SANDWICH COMPOSITES SUBJECT TO MOISTURE DIFFUSION
}

\author{
A Thesis \\ by \\ NIKHIL P. JOSHI \\ Submitted to the Office of Graduate Studies of \\ Texas A\&M University \\ in partial fulfillment of the requirements for the degree of \\ MASTER OF SCIENCE
}

August 2008

Major Subject: Mechanical Engineering 


\title{
ANALYSES OF DEFORMATION IN VISCOELASTIC SANDWICH COMPOSITES SUBJECT TO MOISTURE DIFFUSION
}

\author{
A Thesis \\ by \\ NIKHIL P. JOSHI \\ Submitted to the Office of Graduate Studies of \\ Texas A\&M University \\ in partial fulfillment of the requirements for the degree of \\ MASTER OF SCIENCE
}

\begin{abstract}
Approved by:
Chair of Committee, Anastasia Muliana

Committee Members, Harry Hogan

Eyad Masad

Head of Department, Dennis L. O’Neal
\end{abstract}

August 2008

Major Subject: Mechanical Enigneering 


\begin{abstract}
Analyses of Deformation in Viscoelastic Sandwich Composites Subject to Moisture

Diffusion. (August 2008)

Nikhil P. Joshi, B.E., Sardar Patel University, India

Chair of Advisory Committee: Dr. Anastasia Muliana
\end{abstract}

Sandwich composites with polymer foam core are currently used in load-bearing components in buildings and naval structures due to their high strength to weight and stiffness to weight ratios, excellent thermal insulation, and ease of manufacturing. During their service time, sandwich composites are exposed to various external mechanical and hygro-thermal stimuli. It is known that the constituent properties of the sandwich composites are greatly influenced by the temperature and moisture fields. For example extreme temperature changes and humid environmental conditions can significantly degrade the stiffness and strength of the polymer foam core. This study analyzes the effect of moisture diffusion on the deformation of viscoelastic sandwich composites, which are composed of orthotropic fiber-reinforced laminated skins and viscoelastic polymeric foam core. It is assumed that the elastic and time-dependent (transient) moduli at any particular location in the foam core depend on the moisture concentration at that location. Sequentially coupled analyses of moisture diffusion and deformation are performed to predict overall performance of the studied viscoelastic sandwich systems. A time and moisture dependent constitutive model is used for the polymer foam core. A time-integration algorithm is developed to link this constitutive 
model to finite element (FE) analyses framework. The overall time-dependent responses of the sandwich composites subject to moisture diffusion are analyzed using 2D plane strain and 3D continuum elements. A 23\% increase in the transverse deformation of the viscoelastic sandwich beam is observed due to the moisture degradation. Experimental data and analytical models available in the literature are used to verify the results obtained from the FE code. Parametric studies on the effects of different diffusivity ratios of skin and core materials on stress, strain and displacement fields have been analyzed. At the initial times the effect of moisture on the field variables is found to be most pronounced in the case with the highest diffusivity ratio. Contributions of moisture dependent elastic and the time-dependent moduli to the overall stress, strain and displacement field have been studied. The structural analysis of the sandwich composite under combined moisture diffusion and mechanical loading for two kinds of problems using FE method is performed to complete the study. 


\section{ACKNOWLEDGEMENTS}

I would like to thank my research advisor, Dr. Anastasia Muliana for her patience, guidance and support over the course of this research. She was a constant inspiration, and her assistance and suggestions were invaluable towards the completion of this work. I would like to extend my gratitude to Dr. Harry Hogan and Dr. Eyad Masad for their helpful comments on the work. I would like to acknowledge and thank the Department of Mechanical Engineering at Texas A\&M University for the various opportunities that have helped to make this journey an educational as well as an enjoyable one.

I would like to dedicate this research work to my family. To my parents, who have always encouraged me to pursue my goals, always succeed, and never admit defeat. From them I have learned to be the best that I can be. Thank you for being a part of my life. 


\section{TABLE OF CONTENTS}

Page

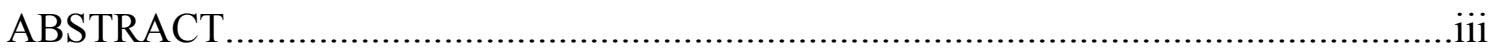

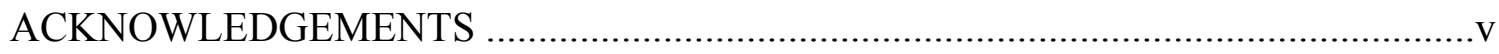

TABLE OF CONTENTS .................................................................................vi

LIST OF FIGURES.........................................................................................

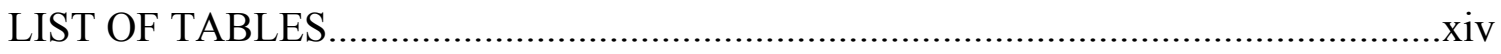

CHAPTER

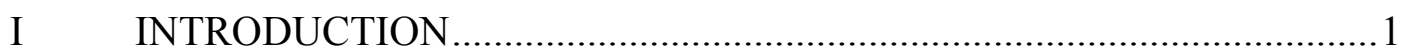

1.1 State of the Art Knowledge in the Effects of Moisture on the Mechanical Behavior of Composites ................................................ 2

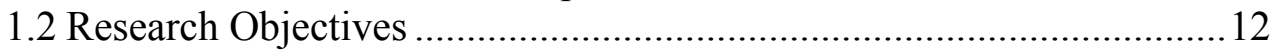

II CONSTITUTIVE MODEL GOVERNING THE DEFORMATION AND THE MOISTURE DIFFUSION IN THE VISCOELASTIC SANDWICH COMPOSITES .............................................................. 15

2.1 Mechanical Response of Viscoelastic Materials ............................... 15

2.2 Governing Equation for Moisture Diffusion Through the Sandwich Composite 25

III SOLUTIONS TO PROBLEM OF COUPLED MOISTURE DIFFUSION AND DEFORMATION IN THE VISCOELASTIC SANDWICH

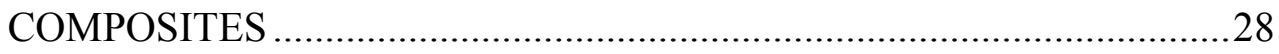

3.1 Analyses of the Moisture Diffusion in the Sandwich Composite ........29

3.2 Analyses of the Deformation in the Viscoelastic Sandwich Composite 39

3.3 Coupled Moisture Diffusion and Deformation in the Viscoelastic

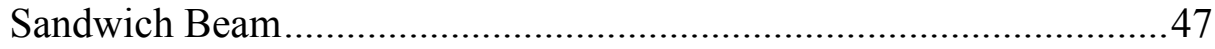

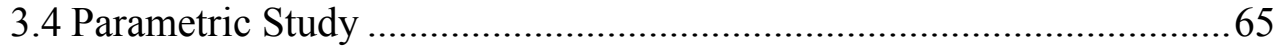


CHAPTER Page

IV FINITE ELEMENT ANALYSIS OF THE SKIN-CORE DELAMINATION AND THE HYGROSCOPIC SWELLING IN SANDWICH COMPOSITES ........................................................... 87

4.1 Delamination at the Skin-Core Interface of the Sandwich

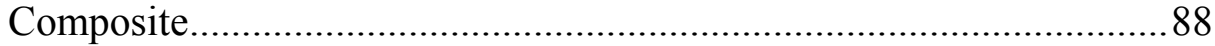

4.2 Hygroscopic Swelling in Sandwich Panels ..................................... 101

V CONCLUSIONS AND FURTHER RESEARCH ............................... 110

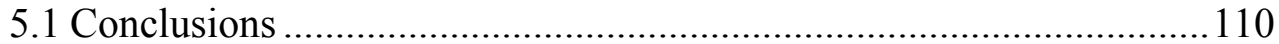

5.2 Further Research .............................................................. 112

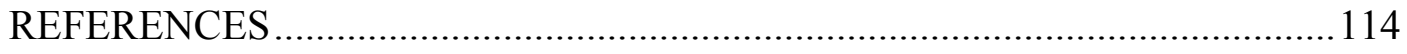

VITA 


\section{LIST OF FIGURES}

FIGURE Page

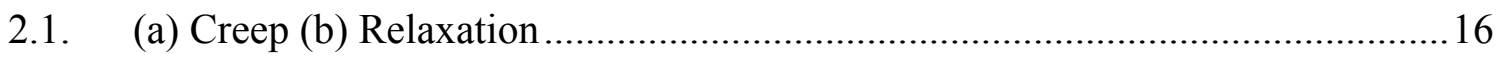

2.2. UMAT incorporating the recursive algorithm to obtain converged solution for each element in the foam core of the sandwich structure .............................24

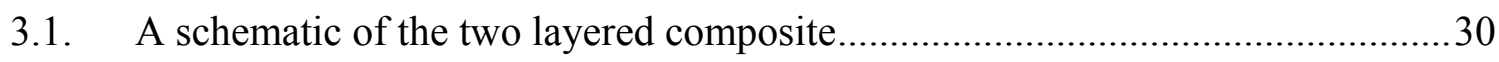

3.2. Evolution of the moisture diffusion profile through the two layered composite

3.3. A schematic of the sandwich composite

3.4. Loading and boundary conditions on the sandwich beam

3.5. Viscoelastic deformation at the midspan

3.6. Deflection at the mid span of the sandwich beam. .44

3.7. Effect of different time increments sizes on instantaneous displacements

3.8. Evolution of concentration of diffusing moisture

3.9. Evolution of concentration of diffusing moisture at a fixed location of $x=0 \ldots \ldots .50$

3.10. Comparison of transverse displacement using 2D and 3D elements at 1200 hours 
FIGURE $\quad$ Page

3.11. Comparison of the transverse displacement field for case (i), case (ii) and case (iii)

3.12. Comparison of the transverse strain field for case (i), case (ii) and case (iii) ......55

3.13. Comparison of the longitudinal displacement field for case (i), case (ii) and case (iii)

3.14. Comparison of the longitudinal strain field for case (i), case (ii) and case (iii)...57

3.15. Comparison of the bending stress field for case (i), case (ii) and case (iii)

3.16. Comparison of the shear strain field for case (i), case (ii) and case (iii)

3.17. Comparison of the shear stress field for case (i), case (ii) and case (iii). 60

3.18. Contour plot of transverse displacement $\left(\mathrm{U}_{2}\right)$ at 1200 hours 61

3.19. Contour plot of axial displacement $\left(\mathrm{U}_{1}\right)$ at 1200 hours. .61

3.20. Contour plot of transverse strain $\left(\mathrm{E}_{22}\right)$ at 1200 hours. 62

3.21. Contour plot of axial strain $\left(\mathrm{E}_{11}\right)$ at 1200 hours .62

3.22. Contour plot of shear strain $\left(E_{12}\right)$ at 1200 hours .63

3.23. Contour plot of bending stress $\left(\sigma_{11}\right)$ at 1200 hours 
$\begin{array}{lll}\text { FIGURE } & \text { Page }\end{array}$

3.24. Contour plot of transverse stress $\left(\sigma_{22}\right)$ at 1200 hours ...........................................64

3.25. Contour plot of shear stress $\left(\sigma_{12}\right)$ at 1200 hours................................................64

3.26. Evolution of the moisture diffusion profile with different diffusivity ratios .......66

3.27. Comparison of the transverse displacement field at the midspan of the sandwich beam for different moisture diffusivity ratios

3.28. Comparison of the axial stress field at a distance of $70 \mathrm{~mm}$ from the midspan of the sandwich beam for different moisture diffusivity ratios

3.29. Comparison of the shear stress field at a distance of $70 \mathrm{~mm}$ from the midspan of the sandwich beam for different moisture diffusivity ratios

3.30. Comparison of the longitudinal displacement field at the midspan of the sandwich beam for different moisture diffusivity ratios

3.31. Comparison of the longitudinal (axial) strain field at the midspan of the sandwich beam for different moisture diffusivity ratios

3.32. Comparison of the transverse strain field at the midspan of the sandwich beam for different moisture diffusivity ratios 75

3.33. Comparison of the shear strain field at a distance of $70 \mathrm{~mm}$ from the midspan of the sandwich beam for different moisture diffusivity ratios

3.34. Creep compliance for the two cases at $\mathrm{x}=13.7 \mathrm{~mm}$ 
FIGURE

Page

3.35. Comparison of the transverse displacement field at the midspan of the sandwich beam for case with moisture dependent instantaneous part and the case with moisture dependent instantaneous and time dependent part 80

3.36. Comparison of the bending stress field at a distance of $70 \mathrm{~mm}$ from the midspan of the sandwich beam for case with moisture dependent instantaneous part and the case with moisture dependent instantaneous and time dependent part

3.37. Comparison of the shear stress field at a distance of $70 \mathrm{~mm}$ from the midspan of the sandwich beam for case with moisture dependent instantaneous part and the case with moisture dependent instantaneous and time dependent part....82

3.38. Comparison of the longitudinal displacement field at the midspan of the sandwich beam for case with moisture dependent instantaneous part and the case with moisture dependent instantaneous and time dependent part

3.39. Comparison of the longitudinal strain field at the midspan of the sandwich beam for case with moisture dependent instantaneous part and the case with moisture dependent instantaneous and time dependent part

3.40. Comparison of the transverse strain field at the midspan of the sandwich beam for case with moisture dependent instantaneous part and the case with moisture dependent instantaneous and time dependent part

3.41. Comparison of the shear strain field at a distance of $70 \mathrm{~mm}$ from the midspan of the sandwich beam for case with moisture dependent instantaneous part and the case with moisture dependent instantaneous and time dependent part....86

4.1. a) DCB test b) TSD test .93 
FIGURE

Page

4.2. A schematic of the sandwich composite glued together with adhesive

.93

4.3. A schematic of the double cantilever beam.

4.4. Cumulative crack length vs. time for a DCB in mode I under creep loading with the crack at the skin-core interface.

4.5. Cumulative crack length vs. time for a DCB in mode I under creep loading with the crack within the glue

4.6. Cumulative crack length vs. time for a TSD beam in mode I under creep loading .98

4.7. Cumulative crack length vs. time for a DCB in mode I under creep loading with the crack at the skin-core interface using the critical failure stress criterion .99

4.8. Von Mises stress plot at the crack tip..... 100

4.9. Finite Element mesh and boundary conditions of the sandwich panel 105

4.10. Hygroscopic strain in the 1-direction (along the fiber) versus time. 105

4.11. Hygroscopic strain in the 2-direction (through the thickness) versus time 106

4.12. Hygroscopic strain in the 3-direction (transverse) versus time 106

4.13. Von Mises stress plot for the sandwich panel 108 
FIGURE $\quad$ Page

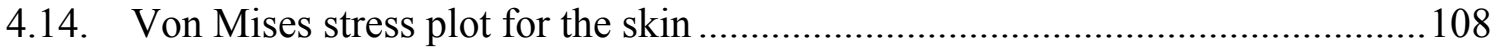

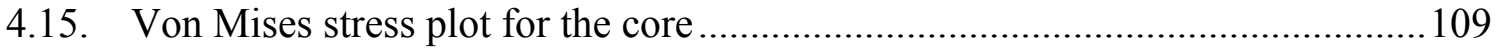




\section{LIST OF TABLES}

TABLE Page

3.1. Material properties and dimensional details of the two layered composite .........33

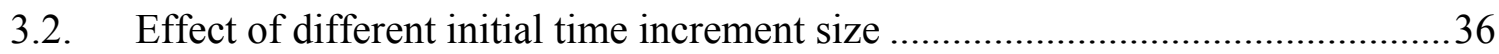

3.3. Effect of different tolerance on the convergence rate .........................................38

3.4. Elastic properties and Prony series coefficients for polyurethane foam core …...42

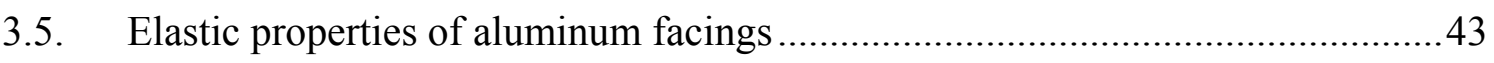

3.6. Elastic properties of Carbon Epoxy laminate and Divinycell H250 ……...........45

3.7. Different moisture diffusivities of the skin and the core ......................................66

4.1. Elastic properties of S2-glass epoxy laminate....................................................

4.2. Elastic properties and Prony series coefficients for FM73M ……………..........92

4.3. The coefficient of moisture expansion of skin and core material .......................104 


\section{CHAPTER I}

\section{INTRODUCTION}

Application of sandwich composites has tremendously increased over a few decades in various industries like automotive, aerospace, marine, etc. Polymeric foam cored sandwich composites are widely used in load-bearing components in buildings such as walls of mobile homes and naval structures because they can provide high strength and stiffness without significantly adding weight and the cost. To improve the reliability in using sandwich composites, it is essential to understand their mechanical responses in terms of stresses and deformations under external mechanical and environmental stimuli. One of the prominent characteristics of polymers is their viscoelasticity. The viscoelastic behavior of polymers is even more aggravated under high stress levels and extreme environmental conditions. However, the effects of combined time-stress-temperature-moisture in the polymer foam on the performance of sandwich structures have not been fully understood. For example, extreme temperature changes and humid environmental conditions can significantly degrade the stiffness and strength of the polymer foam core, which intern degrades the performance of the entire sandwich structure.

This study analyzes the effect of moisture concentration on the deformation of viscoelastic sandwich composites, which are composed of orthotropic fiber-reinforced laminated skins and viscoelastic polymeric foam core. It is assumed that the elastic and

This thesis follows the style of Composite Science and Technology. 
time-dependent (transient) moduli at any particular location in the foam core depend on the moisture concentration at that location. To predict overall performance of the studied viscoelastic sandwich systems, sequentially coupled analyses of diffusion of fluid and deformation are performed. Time and moisture dependent viscoelastic model is used for the polymeric foam core. The generalized single integral constitutive relation for the linear viscoelastic material is modified to include the time-stress-moisture dependence. A time-integration algorithm is developed to link this constitutive model to finite element (FE) analyses framework.

This chapter presents a literature review of analytical, numerical, and experimental works on effects of moisture concentration on polymer based sandwich composites, followed by research objectives.

\subsection{STATE OF THE ART KNOWLEDGE IN THE EFFECTS OF MOISTURE ON THE MECHANICAL BEHAVIOR OF COMPOSITES}

\subsubsection{THE EFFECT OF MOISTURE ON THE MECHANICAL RESPONSE OF LAMINATED COMPOSITE MATERIALS}

External temperature and moisture play important roles in determining the long term behavior of structures made of polymer matrix composite. There is some concern, however, that the mechanical properties of such materials may suffer when the material is exposed to moisture for a long period of time. In general, moisture can affect chemical, electrical, and physical properties of materials, such as strength, modulus, fatigue life, glass transition temperature, etc. Hence, it becomes an issue of prime 
interest to understand the effect of moisture on these performance characteristics of composites.

Springer and Shen [1] conducted experiments to reveal the significant influence of moisture on ultimate tensile strength of Thornel 300/Fiberite 1034 graphite epoxy composites with different fiber orientations. For composites with $0^{\circ}, 45^{\circ}$ fiber orientation and moisture content (weight gain) above 1\%, their ultimate tensile strength reduced by about $20 \%$, while the ultimate tensile strength of composite along the transverse fiber orientation $\left(90^{\circ}\right)$ was reduced by about 60 to $90 \%$. Springer et al. [2] evaluated the effect of moisture on the ultimate tensile strength, tensile modulus, short beam shear strength and shear modulus. Glass Fiber Reinforced Polyester and Vinylester Composites (SMC-R25, SMC-R50, and VE SMC-R25) subjected to different relative humidity showed variations in both strength and modulus. Efforts were also made to evaluate permanent degradation of material properties on drying the specimen and performing the test again on the dried specimen. It was then concluded that some, but not all, of the losses in strength and modulus are recovered. Thus drying out the material does not restore either the strength or the modulus to their original values creating a permanent loss in the material properties. Antoon and Koeing [3] used Fourier Transform infrared spectra to show that the mechanical properties of glass reinforced epoxy composites are irreversibly degraded when exposed to moist environment. Burcham et al. [4] conducted experiments on graphite/polyimide composites to show that the glass transition temperature $\left(\mathrm{T}_{\mathrm{g}}\right)$ is degraded with the increasing moisture concentration. The $T_{g}$ is recovered on drying the specimen. It was 
also observed that permanent toughness losses were observed in samples conditioned at room-temperature and $75 \%$ and $100 \%$ relative humidity $(\mathrm{RH})$ environments indicating some local damage mechanism behind it.

Experiments conducted by Springer and Shen [5] show that the elastic moduli viz. tensile, compression, buckling are also affected by moisture concentration. The buckling moduli of Thornel 300/Fiberite 1034 graphite epoxy composites were measured at temperature ranging from $105{ }^{\circ} \mathrm{K}$ to $422{ }^{\circ} \mathrm{K}$ and at moisture contents ranging from $0 \%$ (dry) to $1.5 \%$ (fully saturated). The measurements were made using $0^{\circ}, 45^{\circ}, 90^{\circ}$ laminates. It was observed that there is a very little impact of moisture on the elastic moduli over the entire spectrum of moisture content from dry to fully saturated for $0^{\circ}$ and $45^{\circ}$ laminates. But for $90^{\circ}$ laminates there was a considerable decrease in the elastic modulus of around 50 to $90 \%$ with the increase in moisture concentration.

Snead and Palazotto [6] performed an analytical investigation to evaluate the stability characteristics of cylindrical, composite graphite/epoxy (AS/3501-5) laminated panels subjected to axial loads under moist environment conditions. The influences of moisture and temperature were investigated by degrading the transverse elastic modulus $E_{2}$ and the shear modulus $G_{12}$, based upon test data for the AS/3501-5 system. Results obtained from the Finite Element Analysis suggested the bifurcation load of the composite panel will degrade with the increasing moisture concentration and temperature. 
Allred [7] studied the effect of moisture on flexural response of Kevlar/Epoxy laminate with $0^{\circ}, 90^{\circ}$ fiber orientations. Two commercially available epoxy resin namely 5208 and CE -9000 reinforced with Kevlar fibers where tested under three point bending test. The experimental results show a reduction in the maximum load carrying capacity and the deflections with the increasing moisture concentration. Substantial changes in the apparent flexural strength were exhibited with the increasing moisture concentration. At $21{ }^{\circ} \mathrm{C}$ and near saturation moisture content, nearly $35-40 \%$ loss in the flexural strength was observed. The load-deflection curves depicted only a $15 \%$ loss in the maximum load carrying capacity. Great differences in the strengths were observed because of the moisture induced swelling that takes place in the laminate thickness together with a $10 \%$ reduction in the stiffness. Because the specimen thickness enters the equations of strength and stiffness as the reciprocal squared and cubed respectively, the reduction in apparent strength and stiffness is greater than the reduction of load carrying capacity. At higher temperatures and higher moisture concentrations these effects were even more pronounced. It was concluded that hygrothermal degradation is linear with increasing temperature and moisture content until the matrix transition temperature ( $\mathrm{Tg})$ is depressed to the test temperature by moisture salvation. Above $\mathrm{Tg}$, the matrix becomes rubbery and the Kevlar filaments do not get enough lateral support leading to the failure at lower load levels due to massive filament buckling. Even at room temperature moisture reduces the strength substantially due to the matrix plasticization effect, which easy out the filament buckling mechanism. 
Gibson et al. [8] analyzed the effects of moisture on the dynamic properties of composites. Chopped fiber composites SMC-R25 and SMC-R65 were subjected to flexural vibration. With the increasing moisture concentration, significant changes in the storage modulus $\left(E^{\prime}\right)$ and loss factor $(\tan \delta)$, which is the ratio of loss modulus $\left(E^{\prime \prime}\right)$ to storage modulus, were noted. An increase of about 500\% in the damping was observed. Efforts were also made to check the reversibility effect of moisture on these properties. It was observed that the moisture induced changes in damping were fully reversible and no permanent structural changes occurred. Adam and Miller [9] suggested two basic mechanisms governing the damping behavior with increasing moisture concentration. In the first mechanism, it is found that increasing the moisture concentrations lowers the glass transition temperature $T_{g}$, dropping of the damping peaks and stiffness drastically, for the temperature increasing through the glass transition range. For operating temperatures below $T_{g}$, a moisture induced reduction in $T_{g}$ would shift the stiffness and damping curves to lower temperatures causing the damping to increase and stiffness to decrease at the operating temperatures. According to the second mechanism, the matrix swelling changes the residual stress distribution, which could increase friction at matrixfiber interfaces, thus increasing damping.

Woldesenbet et al. [10] analyzed the effect of high strain rate on the mechanical properties of polymer matrix composite immersed in water. In contrast to the quasistatic strains, the high strain rates (500 to 1000 per second) had opposite effects on the mechanical properties of the composite fully saturated with moisture. The ultimate strength of fully saturated carbon-epoxy composite specimens was found to increase by 
$10-25 \%$ along the fiber direction with the increasing strain rate as compared to the dry specimen. The effect was even more pronounced along the other two directions, indicating an increase of approximately $30-40 \%$ in the ultimate strength with the increase in the strain rate. This effect was attributed to the fact that plasticization of the matrix is clearly the dominating factor in high strain rate properties when materials are partially or fully wet.

Bouadi and Sun [11] combined the theoretical and experimental work concerned with predicting the hygrothermal effects on the stress field of some laminated composite plates under hygothermal loading. Three coefficients of moisture expansion $\left(\beta_{1}, \beta_{2}, \beta_{12}\right)$ expressed in terms of fiber and matrix properties were used in determining the moisture induced swelling (strains). FE method was used to estimate the magnitude of hygrothermal stresses in laminated composites. It was observed that hygrothermal loading induced very high stresses in cross ply laminates. Whitney and Ashton [12] analyzed the effect of expansional strains (swelling) on vibration, buckling and bending behaviors of composite laminates. Generalized Duhamel-Neumann form of Hooke's law was used in conjunction with classical laminated plate theory. Analytical solution was developed to incorporate the effect of expansional strains due to moisture absorption. The results showed that inplane stresses are induced in plate with edge constrains due to the matrix swelling, which reduces fundamental vibration frequencies and increases deflections in plate subjected to lateral loads. 


\subsubsection{EFFECT OF MOISTURE ON THE VISCOELASTIC BEHAVIOR OF POLYMERS AND POLYMER MATRIX COMPOSITES}

The effect of moisture on the mechanical and physical properties of polymers is significant. Polymers, such as polyester resins, vinylester resin, and epoxy resins, are generally used as binders (matrix) in composite materials. Polymeric foams such as polyurethane, divinycell, and honeycomb, etc are used as core in many sandwich composites. Several experiments have been performed to analyze the effects of moisture on the viscoelastic behavior of these polymers.

Aiello et al. [13] evaluated the effect of moisture on the deformability of vinylester binders (resin). It is observed that the creep strain for the specimen with around $1.42 \%$ of moisture content increased by around $5 \%$ as compared to the dry specimen. Significant changes were also observed in the elastic and dynamic moduli of the moisture absorbed specimen. About $10 \%$ decrease in the dynamic modulus for the specimen with $1.86 \%$ of moisture content was observed. Plushchik and Aniskevich [14] showed that the creep strains are highly sensitive to environmental effects such as moisture and temperature in polyester resins. For a fully saturated material after an hour creep, the strain exceeds almost four times as compared to the dry one. The creeping activation under moisture is described using the method of time-moisture superposition. The short-term creep data was used to analyze the long term creeping effects. Zheng et al. [15] presented the moisture-time superposition principle for polymer nylon-6, analogous to the temperature-time superposition principle previously established. Experiments were also conducted which show that equivalence between moisture and 
service time exists which affect the mechanical properties. Experimental results of creep testing were also used to determine the shift factor and shift function for nylon-6. Harper et al. [16] investigated the effect of moisture on the stress relaxation in Kapton polyimide films. Experiments were conducted to determine relaxation modulus as a function of time, temperature and moisture which was found to be decreasing with increasing temperature and moisture concentration. Analytical model was also developed to describe the effect of temperature, moisture and strain on the relaxation modulus. Of the horizontal and the vertical shift factors the latter was relatively minor and was found to depend only on the relative humidity.

Moisture also plays a significant role in affecting the viscoelastic properties of polymer based composite materials. Flaggs and Crossman [17] explored the capability of the linear viscoelastic material model based upon single hereditary integral formulation to describe the response of polymeric composite laminates subjected to coupled hygrothermal and mechanical stimuli. The hereditary integrals were integrated exactly using an incremental solution procedure, in which temperature, moisture, and external boundary conditions were assumed to remain constant during the viscoelastic time steps $(\Delta \mathrm{t})$. Changes in the hygrothermal conditions and/or boundary conditions are made in elastic steps during which time is held constant $(\Delta \mathrm{t}=0)$. The model predicted that the viscoelastic stress relaxation during complete moisture absorption-desorption cycle at constant temperature leads to a nonrecoverable dimensional and in-plane stress changes in a quasi-isotropic composite laminate. 
Bothelho et al. $[18,19]$ performed experiments to analyze the effect of moisture on the dynamic mechanical properties obtained by free vibration damping test of glass fiber/epoxy composites. It was observed that as a result of the matrix plasticization with the increasing moisture concentration, the natural frequencies and stiffness of all the tested samples decreased and the damping factor increased. The vibration damping curves showed that the maximum peaks of the amplitude decay exponentially as a function of time, also the amplitude decay are more pronounced with the increasing moisture concentration. It was observed that there was a loss of around $19 \%$ in the storage modulus $\left(\mathrm{E}^{\prime}\right)$ at the saturated moisture condition. Loss modulus (E") increased by approximately three times from dry to fully saturated state indicating that the wet specimen dissipates more energy per damping cycle then the dry specimen. Fraga et al. [20] analyzed the dynamic mechanical properties of the unsaturated polyester and vinylester glass fiber composites with a dynamic mechanical analysis (DMA) instrument in three-point bending mode. The samples were dried prior to conducting the experiments in order to determine the aging produced by the water immersion. The loss tangent $(\tan \delta)$ values decrease with the increase in moisture concentration. The storage moduli (E') were measured at two stages: glass stage $\left(\mathrm{T}_{\mathrm{g}}-50 \mathrm{~K}\right)$ and rubbery stage $\left(\mathrm{T}_{\mathrm{g}}+50 \mathrm{~K}\right)$. The glass transition temperature was found to increase after the immersion in the hot water due to the extraction of soluble making the matrix more rigid. $E^{\prime}$ in the rubber state of before immersion is lower than after immersion indicating that the crosslinking density increases after immersion and part of the material is extracted. 


\subsubsection{EFFECT OF MOISTURE ON THE RESPONSE OF SANDWICH SYSTEMS}

Sandwich composites are generally made up of fiber reinforced laminated skins and polymeric core. Major progress has been made on understanding the environmental effects on the response of polymers and laminated composites. It is necessary to understand the effect of the environmental factors like temperature and moisture on these laminates and polymeric foam when glued together as a sandwich composite. Limited work has been done till date to understand the effect of environmental condition on the sandwich composites.

Doxsee et al. [21] described the procedure for calculating the inplane deformation and curvature changes in the sandwich panels composed of honeycomb core and fiber reinforced laminated skin, due to non-uniform temperature and moisture distributions. It was assumed that all the material properties were independent of temperature and moisture concentration. Classical lamination theory was used to predict the inplane deformations and curvature. Granville [22] conducted experiment to carry out a comparative study on the effects of moisture and temperature on the performance of sandwich composites composed of polymethacrylimide (PMI) foams and honeycomb foams glued to glass/epoxy facesheets. Work was intended to understand the effects of moisture on structural, dimensional stability, weight gain and peel strength of sandwich composites. It was observed that moisture absorbed by the PMI foam core sandwich structure was significantly higher than the honeycomb core panels. However the torque required for peeling did not suffer much due to the migration of moisture. Morganti et 
al. [23] also analyzed the effects of moisture on the dimensional stability of sandwich composites. It was concluded that moisture and temperature affects the physical behavior of the composite directly by modifying its structural characteristics such as degrading the matrix properties and inducing microcracks between fibers and matrix etc. The geometric stability of is affected by the "sweeling" phenomenon attributed to the moisture absorption and can induce stress effects.

\subsection{RESEARCH OBJECTIVES}

This study analyzes the effect of moisture diffusion on the deformation of viscoelastic sandwich composites, which are composed of orthotropic fiber-reinforced laminated skins and viscoelastic polymeric foam core. Time and moisture dependent constitutive model is used for the polymer foam core. It is assumed that the elastic and time-dependent (transient) moduli at any particular location in the foam core depend on the moisture concentration at that location. The effects of moisture on the elastic and time-dependent material properties are incorporated, which allow predicting timedependent responses under general stress-moisture loading histories. Sequentially coupled analyses of moisture diffusion and deformation are performed to predict overall time-dependent performance of the studied sandwich systems. A time-integration algorithm is developed to link this constitutive model to finite element (FE) analyses framework. In this study, the integrated material-structural analyses using FE Method are performed on a three point bend test specimen under combined moisture-mechanical loadings. Furthermore, the experimental data and analytical models available in the literature are used to verify the results obtained from the FE code. Parametric studies on 
the effects of different diffusivity ratios of skin and core materials on stress, strain and displacement fields have been analyzed. Contributions of moisture dependent elastic and the time-dependent moduli to the overall stress, strain and displacement field has been studied. In the final section of this study, structural analysis of the sandwich composites under combined moisture diffusion and mechanical loading for two kinds of problems using FE method is presented.

Chapter II presents constitutive material models for the linear isotropic viscoelastic responses of the foam core material and the moisture diffusion in the sandwich composites. The single integral constitutive relation used to model the viscoelastic materials is generalized to multiaxial (3D) constitutive relations of isotropic materials by applying the integral model separately for the deviatoric and volumetric strain-stress relations. It is assumed that the material moduli of the polymeric foam core are functions of moisture concentration. The unsteady Fick's law of diffusion is used to model the moisture diffusion process through the sandwich composite. Constant moisture diffusivity independent of the moisture concentration and stress level at any time is assumed.

Chapter III presents FE results to the problem of coupled moisture diffusion and deformation in the viscoelastic sandwich composite. The FE analysis is performed using $2 \mathrm{D}$ and $3 \mathrm{D}$ solid continuum elements. With $2 \mathrm{D}$ elements plane strain condition is imposed to solve for the deformation in the viscoelastic sandwich beam. Analytical solutions to the problems of moisture diffusion and deformation in the $2 \mathrm{D}$ viscoelastic sandwich composite are also developed in order to verify the results obtained from FE 
code. FE analysis of coupled moisture diffusion and deformation of the viscoelastic sandwich beam under three point bending test is presented. It is assumed that the material moduli of the viscoelastic foam core degrade linearly with moisture concentration. Parametric study is performed to evaluate the effect of different diffusivity ratios of the skin to the core. Finally, the effect of moisture dependent elastic and transient moduli of the viscoelastic foam core on the overall behavior of the sandwich beam is analyzed.

Chapter IV presents structural analyses of the sandwich composites under the combined moisture diffusion and mechanical loading. Two kinds of problems are studied. In the first problem, the effect of moisture diffusion on the delamination between the skin-core interface in a sandwich beam under double cantilever beam (DCB) and tilted sandwich debond (TSD) tests is analyzed. The skin and core are glued together by the polymeric adhesive, whose modulus is assumed to degrade with moisture concentration. The second problem deals with the hygroscopic swelling induced due to moisture diffusion in the sandwich panel. A sandwich panel fixed at the four sides is subjected to moisture diffusion to analyze the stresses and strains induced due to hygroscopic swelling.

Conclusion and further research are stated in Chapter V. 


\section{CHAPTER II}

\section{CONSTITUTIVE MODEL GOVERNING THE DEFORMATION}

AND THE MOISTURE DIFFUSION IN THE VISCOELASTIC

\section{SANDWICH COMPOSITES}

This chapter presents constitutive material models for linear isotropic viscoelastic responses of the foam core material in the sandwich composites and the moisture diffusion in sandwich systems. The sandwich composites are made of linear elastic fiber reinforced laminated skins and viscoelastic polymer foam core. A single integral constitutive relation is used to model viscoelastic materials. The single integral model is generalized to multiaxial (3D) constitutive relations of isotropic materials by applying the integral model separately for the deviatoric and volumetric strain-stress relations. The material moduli at a particular time are functions of moisture concentration at that instant time and independent of the history of moisture diffusion. The moisture diffusion process through the sandwich structure is assumed to be governed by the unsteady Fick's law. It is assumed that the moisture diffusivities of the skin and the core are independent of moisture concentration and stress level at any time.

\subsection{MECHANICAL RESPONSE OF VISCOELASTIC MATERIALS}

Viscoelastic response of a material possesses characteristics of elastic solid and viscous fluid. When subjected to mechanical stimuli, an instantaneous response from the elastic solid followed by a delayed response (time dependent) from the viscous fluid is observed. Several analogs mechanical models involving various spring and dashpot arrangements have been developed to explain the viscoelastic response of the material. 
There are several methods to characterize the material parameters in viscoelastic materials such as creep test, relaxation test, dynamic mechanical loading (DMA). The Fig. 2.1 (a) shows the typical response of a viscoelastic material subjected to a constant stress state (creep load) which is instantaneously increased to $\sigma_{0}$ at time $\mathrm{t}=0$ and then held constant. The strain response comprises of an instantaneous elastic part (OA) and a time dependent $(\mathrm{ABC})$ part. This continued straining or flow under the constant stress is called creep. The Fig. 2.1 (b) shows the typical response of a viscoelastic material subjected to a constant strain state which is instantaneously increased to $\varepsilon_{0}$ at time $\mathrm{t}=0$ and then held constant. In response the stress instantaneously jumps to a value (OA). In order to maintain the constant strain $\varepsilon_{0}$ the stress gradually decreases with time (ABC). For the viscoelastic solid material, this stress decreases asymptotically to some non-zero residual value. This decrease of stress at constant strain value is called stress relaxation.
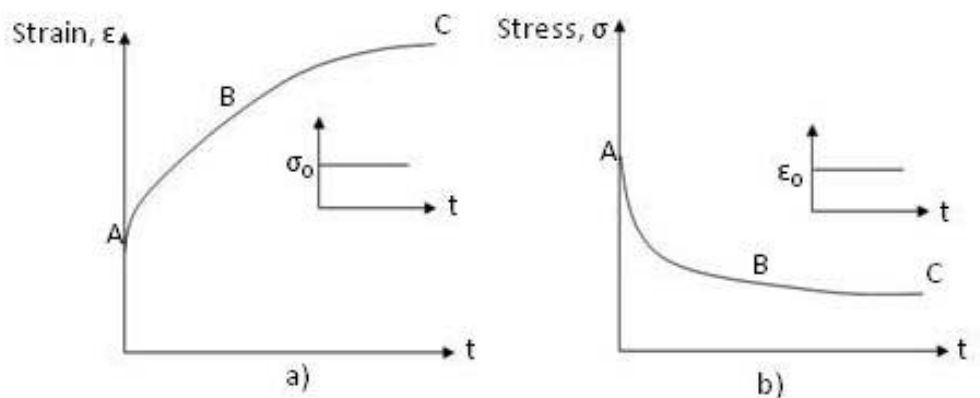

b)

Fig. 2.1. (a) Creep (b) Relaxation 
The DMA essentially characterizes the behavior of viscoelastic polymers under dynamic loading. The storage modulus obtained from the tests represents the elastic component of the material behavior. Whereas the loss modulus is associated with the non recoverable part of the mechanical energy dissipated. The testing is carried out over a range of temperature and loading frequency so as to obtain these properties as functions of temperature and loading rate. The response of the viscoelastic materials is also dependent on temperature and moisture conditions under which they operate. For a class of materials, called the Thermorheologically Simple Materials (TSM), a change in temperature is equivalent to a shift of the behavior on the time or frequency axis (timetemperature superposition). In order to determine the shift factor, which characterizes the material behavior, a set of creep, relaxation, or dynamic tests at different isothermal temperatures is performed. For the viscoelastic materials exhibiting a nonlinear response, a single creep or relaxation test is not sufficient to characterize the material response. A series of creep test at different stress levels or relaxation test at different strain levels are required to characterize the non linear material properties. If the obtained stain responses or stress responses follow the principle of superposition and linear scaling, then the material is categorized as a linear viscoelastic material or else the material belongs to as a non-linear viscoelastic material. For an applied step (creep) stress changed by a factor $\alpha$ if the corresponding strain response is changed by the same factor $\alpha$ then it implies that scaling is satisfied. If the strain history induced when subjected to multistep stress history can be obtained by superposition of responses to each step considered separately or vice versa then the material is said to have satisfied 
the principle of superposition. Another important feature governing the viscoelastic response is the history of the loading and time when the load is applied.

The solution to the problem in linear viscoelasticity (i.e. the field variables such as stress, strain and displacements) can be obtained directly from the corresponding linear elasticity problem by means of Correspondence Principle also called as elasticviscoelastic analogy as discussed by Rajagopal and Wineman [24] and Christenen [25]. The principle incorporates the use of Laplace transform applied to the elasticity solution assuming that the Laplace transform of all the time variables exists. The Laplace transformed viscoelastic solution is obtained directly from the solution of the corresponding elasticity problem by replacing the elastic material properties by the Laplace transformed viscoelastic properties multiplied with the Laplace transform variable and reinterpreting the elastic field variable as corresponding Laplace transformed viscoelastic field variables. The final solution is then obtained on inverting the transformed solution. However, for the Correspondence Principle to work certain restrictions on the boundary conditions must be satisfied. The interface between boundaries under prescribed traction and boundaries under prescribed displacement must not change with time, although the loads and displacements may be time dependent.

As discussed by Rajagopal and Wineman [24] for certain class of problems the Correspondence Principle does not work. As per the discussion, if all the material points of the body under consideration are not a part of the body for all the times $t \geq 0$ then the Correspondence Principal breaks. Also, for a fixed point on the boundary, if the boundary condition is changed from one specifying a displacement to one specifying a 
surface traction during the deformation then the Correspondence Principle is no more applicable. Lee and Rogers [26] discussed the solution of stress and strain fields for more general viscoelastic materials that are represented by general hereditary integral stress strain relations. These stress and strain relations were deducted directly by superposition of relaxation and creep responses. The stress-strain relations are expressed by convolution integral form and so more general problems that are beyond the scope of Laplace Transform method could be solved by this method.

\subsubsection{GENERALIZED SINGLE INTEGRAL CONSTITUTIVE RELATION FOR THE LINEAR ISOTROPIC VISCOELASTIC MATERIAL}

The sandwich composites are generally made up of metallic or fiber reinforced laminated skins and polymeric cores. These polymeric cores can exhibit significant viscoelastic responses. In this study a generalized single integral constitutive relation is used to model the linear viscoelastic behavior of the polymeric core of the sandwich structure. The general single integral constitutive relation for a linear viscoelastic material under uniaxial loading (non aging) is written as:

$$
\varepsilon^{t} \equiv \varepsilon(t)=D_{0} \sigma^{t}+\int_{0}^{t} \Delta D(t-\tau) \frac{d \sigma^{\tau}}{d \tau} d \tau
$$

Where $\mathrm{D}_{0}$ and $\Delta \mathrm{D}$ are the uniaxial instantaneous and transient creep compliances. As discussed in the Chapter I moisture is found to have a pronounced effect on the material moduli of viscoelastic materials. This dependence of the material moduli on the moisture concentration is incorporated by modifying the Eq. (2.1) which is then written as: 


$$
\varepsilon^{t} \equiv \varepsilon(t)=g_{0}(c) D_{0} \sigma^{t}+g_{1}(c) \int_{0}^{t} \Delta D(t-\tau) \frac{d \sigma^{\tau}}{d \tau} d \tau
$$

Here $\mathrm{g}_{0}$ measures the increase or decrease in the instantaneous compliance with the change in moisture concentration and the parameter $g_{1}$ measures the increase or decrease in the transient compliance with the change in moisture concentration.

This uniaxial viscoelastic relation in Eq. (2.2) is generalized for multiaxial (3D) constitutive relations of isotropic materials [27] by separating the deviatoric and volumetric stress-strain relations as:

$$
\begin{aligned}
& \varepsilon_{i j}^{t}=e_{i j}^{t}+\frac{1}{3} \varepsilon_{k k}^{t} \delta_{i j}^{t} \\
& e_{i j}^{t}=\frac{1}{2} g_{0}(c) J_{0} S_{i j}^{t}+\frac{1}{2} g_{1}(c) \int_{0}^{t} \Delta J(t-\tau) \frac{d S_{i j}^{\tau}}{d \tau} d \tau \\
& \varepsilon_{k k}^{t}=\frac{1}{3} g_{0}(c) B_{0} \sigma_{k k}^{t}+\frac{1}{3} g_{1}(c) \int_{0}^{t} \Delta B(t-\tau) \frac{d \sigma_{k k}^{\tau}}{d \tau} d \tau
\end{aligned}
$$

Here $e_{i j}, \varepsilon_{k k}, S_{i j}$ and $\sigma_{k k}$ are used to denote the deviatoric strains, volumetric strain, deviatoric stress and volumetric stress respectively. The parameters $\mathrm{J}_{0}$ and $\mathrm{B}_{0}$ are the instantaneous elastic shear and bulk compliances, respectively. The transient shear and bulk compliances are represented by $\Delta \mathrm{J}$ and $\Delta \mathrm{B}$, respectively. There are quite a few ways in which these transient parts can be expressed such as the power series, rational polynomial form, Prony series etc. Here, a Prony series (series of exponential functions) is used for the transient part owing to the advantage this representation provides in solving the integral form in Eq. (2.3) recursively. The parameters of the multi-axial behaviors are modeled as a function of the current moisture content $c^{t}$. The matrix 
Poisson's ratio, $v$, is assumed to be time independent, as a result of which the shear and bulk compliances are expressed as:

$$
\begin{array}{ll}
J_{0}=2(1+v) D_{0} & B_{0}=3(1-2 v) D_{0} \\
\Delta J^{t}=2(1+v) \Delta D^{t} & \Delta B^{t}=3(1-2 v) \Delta D^{t}
\end{array}
$$

Where the uniaxial transient compliance in terms of the Prony series coefficients is expressed as:

$$
\Delta D^{t}=\sum_{n=1}^{N} D_{n}\left(1-\exp \left[-\lambda_{n} t\right]\right)
$$

Here $\mathrm{N}$ is the number of terms, $D_{n}$ is the $n^{\text {th }}$ coefficient of the Prony series, and $\lambda_{n}$ is the $n^{\text {th }}$ reciprocal of creep time.

Using the Eqs. (2.4) and (2.5), the deviatoric and volumetric strains in Eq. (2.3) can be written as:

$$
\begin{aligned}
& e_{i j}^{t}=\frac{1}{2} g_{0}^{t} J_{0} S_{i j}^{t}+\frac{1}{2} g_{1}^{t} S_{i j}^{t} \sum_{n=1}^{N} J_{n}-\frac{1}{2} g_{1}^{t} \sum_{n=1}^{N} J_{n} q_{i j, n}^{t} \\
& \text { where } q_{i j, n}^{t}=\int_{0}^{t} \exp \left[-\lambda_{n}(t-\tau)\right] \frac{d S_{i j}^{\tau}}{d \tau} d \tau \\
& \varepsilon_{k k}^{t}=\frac{1}{3} g_{0}^{t} B_{0} \sigma_{i j}^{t}+\frac{1}{3} g_{1}^{t} \sigma_{k k}^{t} \sum_{n=1}^{N} B_{n}-\frac{1}{3} g_{1}^{t} \sum_{n=1}^{N} B_{n} q_{k k, n}^{t} \\
& \text { where } q_{k k, n}^{t}=\int_{0}^{t} \exp \left[-\lambda_{n}(t-\tau)\right] \frac{d \sigma_{k k}^{\tau}}{d \tau} d \tau
\end{aligned}
$$

These integral forms in Eqs. (2.6) and (2.7) are called the Stieltjes convolution. The solutions to these integrals are generally obtained by utilizing Laplace transforms. In certain cases where it is not feasible or almost impossible to obtain a closed form solution of the integral, a numerical approach is often employed. One of the numerical 
approaches known is the recursive integration method. Taylor et. al. [28] used the recursive method to solve the $1 \mathrm{D}$ viscoelastic integral model. In this study, the recursive approach is used to solve the integral parts in Eqs. (2.6) and (2.7). A recursive method allows for developing incremental formulation and integration of the current stress state based on the given time, strain, and moisture increments and the history variables stored at the previous time step. As illustrated in Eq. (2.8), the trial incremental strain tensor $\Delta \varepsilon_{i j}^{t}$ and moisture $\Delta c^{t}$ are obtained at each iteration within the incremental time-step $\Delta t$.

The goal is to calculate the current total stress $\Delta \sigma_{i j}^{t}$ and the material's consistent tangent stiffness $C_{i j k l}^{t}$ from given current variables and history variables stored from the previous converged solution at time $(t-\Delta t)$. The converged $C_{i j k l}^{t}$ at the current time $t$ will be used to provide incremental trial strains for the next time step $(t+\Delta t)$. This procedure in Eq. (2.8) is performed at every material (Gaussian) integration point within each element at each structural iteration to achieve structural and material convergence simultaneously as shown in Fig. 2.2. 
Given: $\Delta \varepsilon_{i j}^{t}, \Delta c^{t}, \Delta t$

$$
\begin{aligned}
& \varepsilon_{i j}^{t}=\varepsilon_{i j}^{t-\Delta t}+\Delta \varepsilon_{i j}^{t} \\
& c^{t}=c^{t-\Delta t}+\Delta c^{t} \\
& t=(t-\Delta t)+\Delta t
\end{aligned}
$$

Calculate: Incremental form

$$
\begin{aligned}
& \sigma_{i j}^{t}=\sigma_{i j}^{t-\Delta t}+\Delta \sigma_{i j}^{t} \\
& \Delta \sigma_{i j}^{t}=f\left(c^{t}, \Delta \varepsilon_{i j}^{t}\right) \\
& C_{i j k l}^{t}=\frac{\partial \Delta \sigma_{i j}^{t}}{\partial \Delta \varepsilon_{i j}^{t}}
\end{aligned}
$$

or Total form

$$
\begin{aligned}
& \sigma_{i j}^{t}=f\left(c^{t}, \varepsilon_{i j}^{t}\right) \\
& C_{i j k l}^{t}=\frac{\partial \sigma_{i j}^{t}}{\partial \varepsilon_{i j}}
\end{aligned}
$$

Thus, the hereditary integrals in Eqs.(2.6) and (2.7) using the recursive integration method are expressed as:

$$
\begin{aligned}
q_{i j, n}^{t} & =\int_{0}^{t-\Delta t} \exp \left[-\lambda_{n}(t-\tau)\right] \frac{d S_{i j}^{\tau}}{d \tau} d \tau+\int_{t-\Delta t}^{t} \exp \left[-\lambda_{n}(t-\tau)\right] \frac{d S_{i j}^{\tau}}{d \tau} d \tau \\
& =\exp \left[-\lambda_{n}(t-\tau)\right] q_{i j, n}^{t-\Delta t}+\left(S_{i j}^{t}-S_{i j}^{t-\Delta t}\right) \frac{1-\exp \left[-\lambda_{n} \Delta h^{t}\right]}{\lambda_{n} \Delta h^{t}} \\
q_{k k, n}^{t} & =\int_{0}^{t-\Delta t} \exp \left[-\lambda_{n}(t-\tau)\right] \frac{d \sigma_{k k}^{\tau}}{d \tau} d \tau+\int_{t-\Delta t}^{t} \exp \left[-\lambda_{n}(t-\tau)\right] \frac{d \sigma_{k k}^{\tau}}{d \tau} d \tau \\
& =\exp \left[-\lambda_{n}(t-\tau)\right] q_{k k, n}^{t-\Delta t}+\left(\sigma_{k k}^{t}-\sigma_{k k}^{t-\Delta t}\right) \frac{1-\exp \left[-\lambda_{n} \Delta h^{t}\right]}{\lambda_{n} \Delta h^{t}}
\end{aligned}
$$


Here the incremental time is expressed as $\Delta h^{t}=t-(t-\Delta t)$. The first part includes the integral with limits $(0, \mathrm{t}-\Delta \mathrm{t})$, i.e. up to the previous time step, which is considered as history variable stored and updated at the end of each time increment. The limits of the second part are taken as $(\mathrm{t}-\Delta \mathrm{t}, \mathrm{t})$, which is the current incremental step. The parameters $q_{i j, n}^{t-\Delta t}$ and $q_{k k, n}^{t-\Delta t}, \mathrm{n}=1 \ldots \mathrm{N}$ are the hereditary integral for every term in the Prony series in the form of deviatoric and volumetric strains. Total number of history variables is $7 \mathrm{xNxNGauss;}$ where 7 is the total number of deviatoric and volumetric components, $\mathrm{N}$ is the number of terms in the Prony series, and NGauss is the number of Gaussian integration points.

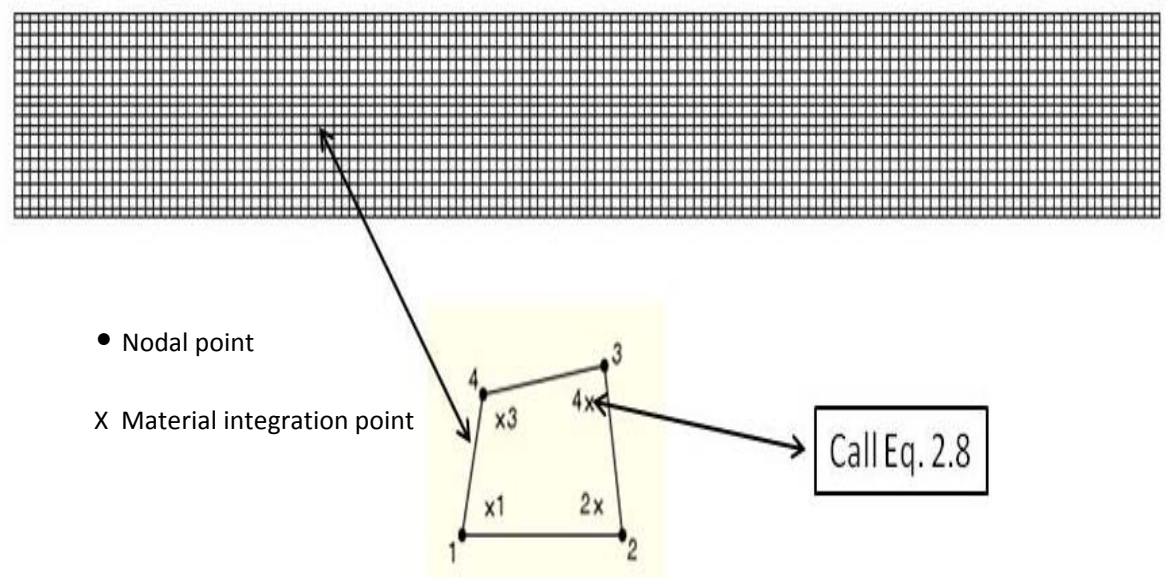

Fig. 2.2. UMAT incorporating the recursive algorithm to obtain converged solution for each element in the foam core of the sandwich structure 


\subsection{GOVERNING EQUATION FOR MOISTURE DIFFUSION THROUGH THE SANDWICH COMPOSITE}

It has been experimentally shown that moisture can degrade the material properties of the composites significantly. It becomes essential to understand the moisture diffusion through the sandwich composite and deformation due to the existence of moisture concentration. To investigate the effect of moisture diffusion on the degradation of the composite materials and its subsequent response, simultaneous study of the problem of moisture diffusion (which is assumed to be governed by unsteady Fick's equation) and deformation of viscoelastic materials is carried out. The equations for the motion of the fluid and the motion of the composite are coupled as the material moduli depend on the concentration of the fluid (moisture).

Diffusion in polymer systems is generally Non-Fickian or anomalous accompanied by damage formation when subjected to external stress. The Non-Fickian diffusion can adequately capture the changes to the polymer system induced by the absorbed penetrant such as chemical degradation, swelling and/or changes induced due to microcracks (damage) etc. Despite the diffusion process being generally NonFickian, in absence of damage or negligible damage, the diffusion of moisture through the composite solids can be assumed to be Fickian. The limitation that the Fickian diffusion model exhibits is that it tends to overestimate the moisture absorption for a short diffusion time. In this specific study it is assumed that the moisture diffusion through the sandwich composite is governed by the unsteady Fick's law of diffusion. The concentration of the fluid at any point $(x, y, z)$ in the sandwich structure and at time 
$\mathrm{t}$ is denoted by $\mathrm{c}=\mathrm{c}(\mathrm{x}, \mathrm{y}, \mathrm{z}, \mathrm{t})$. The rate of the diffusing fluid related to the concentration gradient through the three dimensional form of Fick's law of diffusion is written as:

$\frac{\partial c}{\partial t}=-\nabla \cdot-\left[\mathbf{D}_{\text {iff }}\right] \nabla c$

Here $\mathbf{D}_{\text {iff }}$ is a second order diffusivity tensor and $\nabla$ denotes the gradient operator. For the case when the components $\mathrm{D}_{\mathrm{ij}}$ of the diffusivity tensor are independent of the spatial coordinates $(\mathrm{x}, \mathrm{y}, \mathrm{z})$ and the moisture concentration at any time (constant diffusivity tensor) the Eq. (2.11) becomes:

$\frac{\partial c}{\partial t}=\mathbf{D}_{\text {iff }} \nabla^{2} c$

In the case when the diffusing fluid is more pronounced in one direction, the three dimensional form of Fick's law of diffusion can be reduced to one dimensional form which is stated as:

$\frac{\partial c}{\partial t}=D_{i f f} \nabla^{2} c$

Here the diffusivity $\mathrm{D}_{\text {iff }}$ is now a scalar. One could incorporate the effect of stress assisted diffusion by allowing the diffusivity D to depend on the stress. The details of this could be obtained from the early study on stress assisted diffusion by Weitsman [29]. Thus the concentration of the fluid is now a function of only one spatial coordinate and time written as $c=c(x, t)$. 
In this study, the moisture concentration at every location and every time throughout the sandwich structure can be obtained by solving Eqs. (2.12) or (2.13) by imposing the initial and boundary conditions. Once the moisture concentration profile throughout the structure is known, the material properties that depend on the moisture concentration, $\mathrm{g}_{0}$ and $\mathrm{g}_{1}$ in Eq (2.2), can be determined and the viscoelastic deformation can be solved. In order to accomplish this a numerical algorithm (UMAT in Abaqus an FE commercial code) is derived based on implicit stress integration solutions within a general displacement based FE structural analyses for small deformations and coupled moisture-mechanical problems. Iterative scheme as stated above is used to obtain solutions at structural and material levels. 


\section{CHAPTER III}

\section{SOLUTIONS TO PROBLEM OF COUPLED MOISTURE}

\section{DIFFUSION AND DEFORMATION IN THE VISCOELASTIC}

\section{SANDWICH COMPOSITES}

In this chapter, the problem of coupled moisture diffusion and deformation in the viscoelastic sandwich composite is analyzed. As discussed in the Chapter I moisture is found to have a significant impact on the behavior of the viscoelastic materials. Therefore, there arises a need to analyze the effect of moisture diffusion when coupled with the deformation in the viscoelastic sandwich composite. A FE approach is used to analyze this problem. The analysis is performed using $2 \mathrm{D}$ and $3 \mathrm{D}$ continuum solid elements. The results obtained by FE code are first validated using the analytical solutions. To begin with, the analytical solution to the moisture diffusion problem in two layered composite is developed for unsteady Fick's law of diffusion. The results obtained from the FE code for the diffusion of moisture in the two layered composite are validated using the analytical solution developed. The two layered composite model provide simpler closed form solutions compared to three layered systems and this solution is used to determine suitable range of time-increments in the FE analyses. Further, the analytical solution to the problem of deformation in the viscoelastic sandwich beam is obtained based on the strength of material approach and Correspondence Prinicple. The analytical solution is to validate responses from the FE analyses in terms of mesh size and time increment convergent studies. 
The later part of the chapter presents the FE analyses of coupled moisture diffusion and deformation of the viscoelastic sandwich beam under three point bending. The material modulus of the viscoelastic foam core, in the coupled problem of moisture diffusion and deformation is assumed to be linearly degrading with moisture concentration. ABAQUS FE software is used to simulate the analyses. The results obtained show the aggravated effect of moisture on the viscoelastic response of the sandwich beam. Parametric studies are performed to understand the effect of different moisture diffusivity ratios of the skin and core in the sandwich beam and the effect of moisture dependent elastic and transient moduli of the viscoelastic foam core on the overall behavior of the sandwich beam.

\subsection{ANALYSES OF THE MOISTURE DIFFUSION IN THE SANDWICH COMPOSITE}

\subsubsection{ANALYTICAL SOLUTION}

As stated in the beginning of the chapter, a FE approach is used to obtain the solution to the moisture diffusion problem through the sandwich composite. Therefore, there arises a need to ensure that the results obtained from the FE code are in good agreement with the analytical solution developed. In order to reduce the complexity in developing the analytical solution to the moisture diffusion problem of the sandwich (three layered) composite, a two layered composite is considered. Fig 3.1 shows all the dimensional details of the two layered composite. Moisture concentration is applied at the surface of the layer 1 as shown in the Fig 3.1. It is assumed that the moisture diffuses from the surface of the layer 1, through the thickness of the composite into the 
layer 2. No other mode of diffusion such as moisture diffusion from the sides of the composite is considered. The problem of moisture diffusion thus reduces to a one dimensional (through the thickness) moisture diffusion problem.

\section{APPLIED MOISTURE CONTENT $c_{0}$}

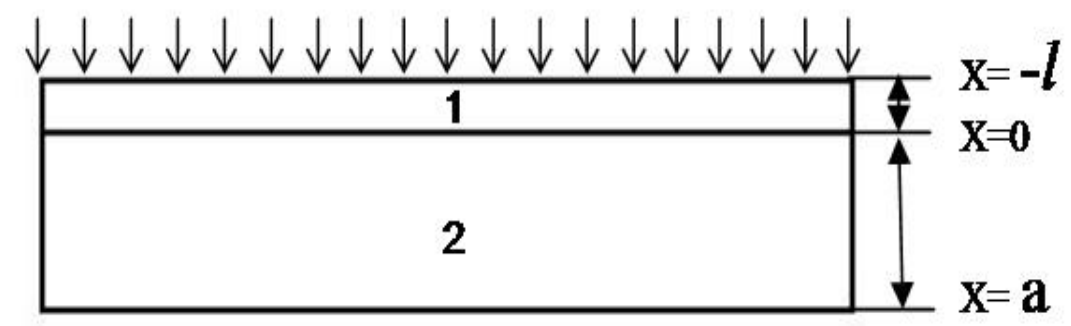

Fig. 3.1. A schematic of the two layered composite

The solution to this problem of moisture diffusion through the two layered composite is derived using the unsteady Fick's law of diffusion as stated in the Eq. (2.13). The solution to the Eq. (2.13) can be obtained by prescribing proper initial and boundary value conditions, which are written as:

$$
\begin{aligned}
& c^{(1)}(-l, t)=C_{0}, \quad t \geq 0 \\
& c^{(2)}(a, t)=0, \quad t \geq 0 \\
& c^{(1)}(x, 0)=0, \quad-l<x \leq 0 \\
& c^{(2)}(x, 0)=0, \quad 0 \leq x \leq a
\end{aligned}
$$


The conditions of moisture concentration and flux of the fluid at the interface being continuous yield the following interfacial conditions:

$$
\begin{aligned}
& c^{(1)}(0, t)=c^{(2)}(0, t), \quad t>0 \\
& D_{i f f}^{(1)} \frac{\partial c^{(1)}}{\partial x}(0, t)=D_{i f f}^{(2)} \frac{\partial c^{(2)}}{\partial x}(0, t), t>0
\end{aligned}
$$

Here the superscripts (1) and (2) denote the layer number as shown in the Fig 3.1. $c^{(i)}$ denotes the moisture concentration at any point in the $i^{\text {th }}$ layer and $D_{i f f}^{(i)}$ denotes the moisture diffusivity of the $i^{\text {th }}$ layer, where $i=1$ or 2 .

The solution to the Eq. (2.13) for a two layered composite using the boundary and the initial condition as in Eqs. (3.1) and (3.2), respectively, is written as:

$$
\begin{aligned}
& c^{(1)}=C_{0}\left[\frac{a D_{i f f}^{(1)}-x D_{i f f}^{(2)}}{a D_{i f f}^{(1)}+l D_{i f f}^{(2)}}\right] \\
& -2 C_{0} \sum_{m=1}^{\infty}\left[\frac{\left\{\cos \left(\beta_{m} x\right) \sin \left(\frac{\beta_{m} a}{R}\right)-R \sin \left(\beta_{m} x\right) \cos \left(\frac{\beta_{m} a}{R}\right)\right\} e^{-D_{i f f}^{(1)} \beta_{m}^{2} t}}{\beta_{m}\left\{\sin \left(\beta_{m} l\right) \sin \left(\frac{\beta_{m} a}{R}\right)(a+l)-\cos \left(\beta_{m} l\right) \cos \left(\frac{\beta_{m} a}{R}\right)\left(\frac{a}{R}+R l\right)\right\}}\right] \\
& c^{(2)}=C_{0}\left[\frac{(a-x) D_{i f f}^{(1)}}{a D_{i f f}^{(1)}+l D_{i f f}^{(2)}}\right] \\
& -2 C_{0} \sum_{m=1}^{\infty}\left[\frac{\sin \left(\frac{\beta_{m}(a-x)}{R}\right) e^{-D_{i f f}^{(1)} \beta_{m}^{2} t}}{\beta_{m}\left\{\sin \left(\beta_{m} l\right) \sin \left(\frac{\beta_{m} a}{R}\right)(a+l)-\cos \left(\beta_{m} l\right) \cos \left(\frac{\beta_{m} a}{R}\right)\left(\frac{a}{R}+R l\right)\right\}}\right] \\
& \text { where, } \quad R=\sqrt{\frac{D_{i f f}^{(2)}}{D_{i f f}^{(1)}}}
\end{aligned}
$$


Here $\beta_{m}$ are the roots of the Eq. 3.4, which is written as:

$$
\cos \left(\beta_{m} l\right) \sin \left(\frac{\beta_{m} a}{R}\right)+R \sin \left(\beta_{m} l\right) \cos \left(\frac{\beta_{m} a}{R}\right)=0
$$

For an applied concentration of $C_{0}=0.5606 \mathrm{gm} / \mathrm{mm}^{3}$ the moisture diffusion profile through the thickness of the two layered composite at various times beginning from time $\mathrm{t}=0 \mathrm{sec}$ to steady state time of $\mathrm{t}=1828 \mathrm{sec}$ is shown in the Fig 3.2. The dimensional details and the required material properties are given in Table 3.1. A Finite Element (FE) analysis is done on the same problem using 2D solid continuum elements (DC2D4 elements form ABAQUS element library). The moisture profiles obtained from the FE analyses are in good agreement with the analytical solution developed in Eq. (3.3).

A similar approach can also be used to obtain the analytical solution to the problem of moisture diffusion for the sandwich composite. Once this moisture diffusion profile is obtained it can then be coupled with the deformation to analyze the effect of moisture concentration on the deformation in the viscoelastic sandwich composite. 


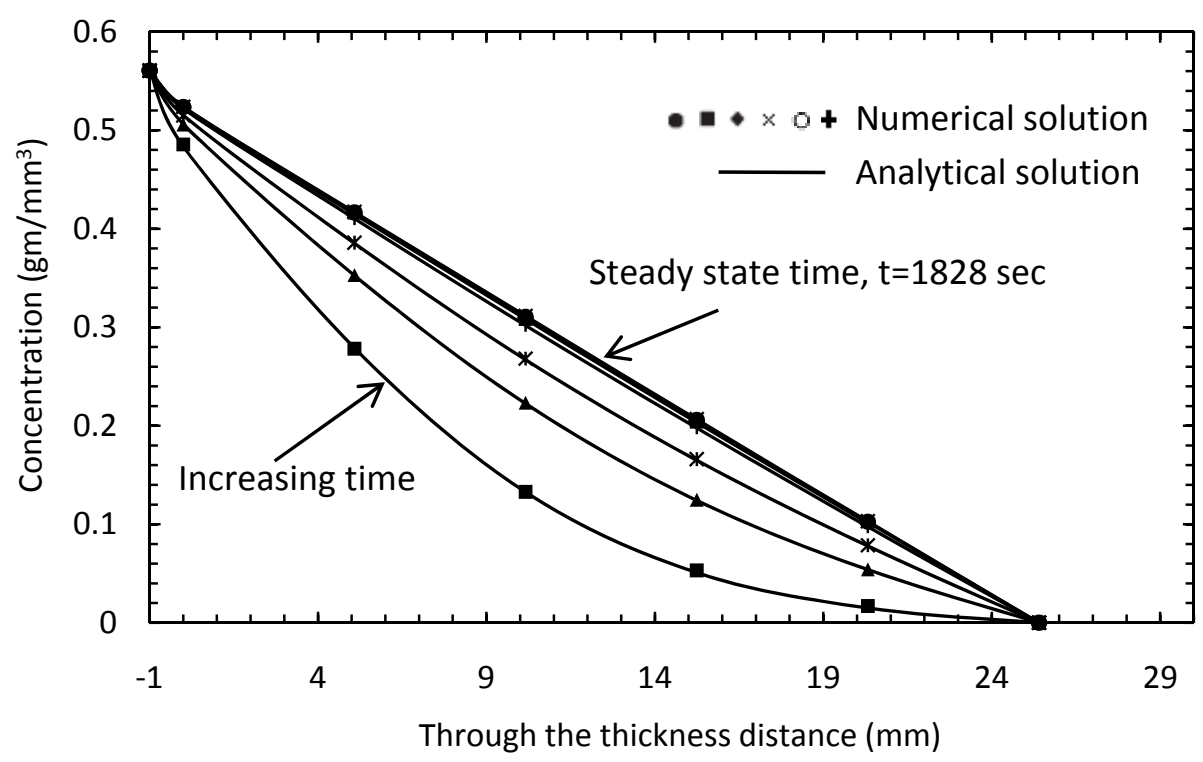

Fig. 3.2. Evolution of the moisture diffusion profile through the two layered composite

Table 3.1. Material properties and dimensional details of the two layered composite

\begin{tabular}{|l|c|}
\hline $\begin{array}{c}\text { Moisture Diffusivity of the first layer } \\
\qquad \begin{array}{c}D_{i f f}^{(1)}\left(\mathrm{mm}^{2} / \mathrm{sec}\right) \\
\text { Moisture Diffusivity of the second } \\
\text { layer } D_{i f f}^{(2)}\left(\mathrm{mm}^{2} / \mathrm{sec}\right)\end{array}\end{array}$ & 0.1144 \\
Thickness of the first layer $l(\mathrm{~mm})$ & 0.2 \\
\hline Thickness of the second layer $a(\mathrm{~mm})$ & 1 \\
\hline
\end{tabular}




\subsubsection{CONVERGENCE STUDY USING FE METHOD}

A sandwich beam $254 \mathrm{~mm}$ long, $27.4 \mathrm{~mm}$ thick, and $25.4 \mathrm{~mm}$ wide is subjected to moisture loading as shown in the Fig 3.3. The skin is carbon epoxy laminate and the core is polyurethane foam with the assumed identical moisture diffusivities of $1.06 \mathrm{E}-2$ $\mathrm{mm}^{2} / \mathrm{sec}$. Moisture concentration of $1 \mathrm{gm} / \mathrm{mm}^{3}$ is applied at the surface of the top skin of the sandwich composite. It is assumed that the moisture diffuses from the surface of the top skin, through the thickness of the sandwich structure to the bottom skin. No other mode of diffusion such as moisture diffusion from the sides of the sandwich structure is considered. The problem of moisture diffusion thus reduces to a one dimensional (through the thickness) moisture diffusion problem.

The solution to this problem governed by the Fick's law of diffusion stated in the Eq. (2.13) can be obtained by prescribing proper initial and boundary value conditions, which are written as:

$$
\begin{aligned}
& c^{(1)}(27.4, t)=1, \forall t \geq 0 \\
& c^{(1)}(x, 0)=0, \forall 26.4 \leq x<27.4 \\
& c^{(2)}(x, 0)=0, \forall 1 \leq x \leq 26.4 \\
& c^{(3)}(x, 0)=0, \forall 0 \leq x \leq 1
\end{aligned}
$$




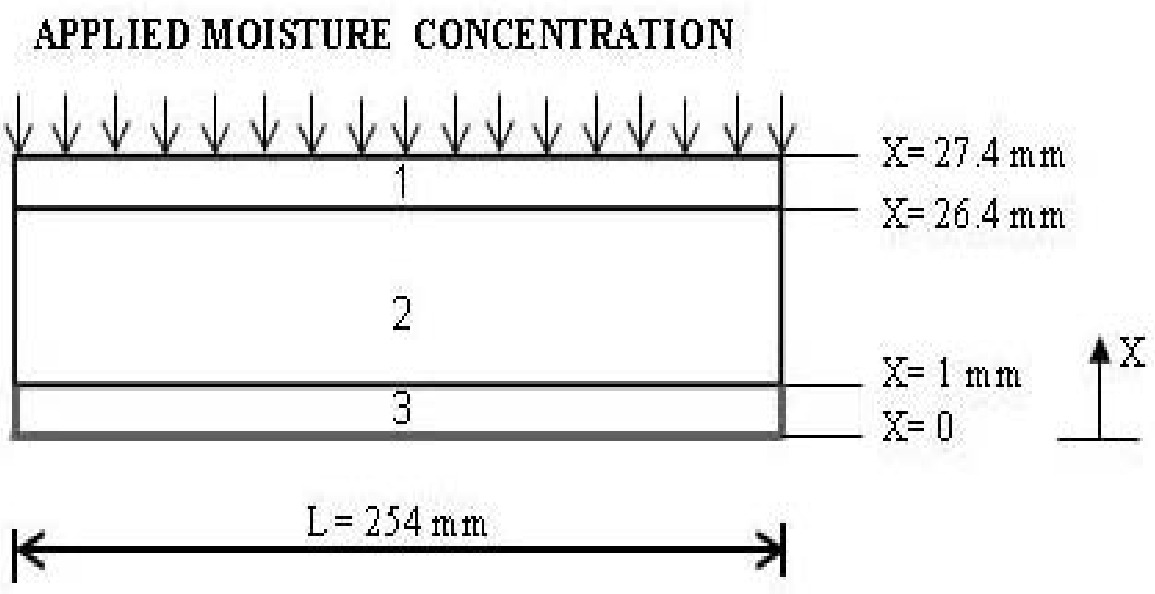

Fig. 3.3. A schematic of the sandwich composite

The continuity of the moisture concentration and the flux of the fluid flow at the interface are written as:

$$
\begin{aligned}
& c^{(1)}(26.4, t)=c^{(2)}(26.4, t), \quad \forall t>0 \\
& c^{(2)}(1, t)=c^{(3)}(1, t), \quad \forall t>0 \\
& D_{i f f}^{(1)} \frac{\partial c^{(1)}}{\partial x}(26.4, t)=D_{i f f}^{(2)} \frac{\partial c^{(2)}}{\partial x}(26.4, t), \quad \forall t>0 \\
& D_{i f f}^{(2)} \frac{\partial c^{(2)}}{\partial x}(1, t)=D_{i f f}^{(3)} \frac{\partial c^{(3)}}{\partial x}(1, t), \forall t>0
\end{aligned}
$$

In this study, FE approach has been used to simulate the moisture diffusion process through the sandwich structure. Therefore, it becomes extremely essential to evaluate the parameters such as the time increment and the tolerance that affect the accuracy of the numerical solution. With the boundary conditions stated in the Eqs. 
(3.5) and (3.6) the steady state is said to have reached when the concentration throughout the sandwich structure reaches a value of $1 \mathrm{gm} / \mathrm{mm}^{3}$. The chosen time increment in the FE analyses can significantly affect the accuracy of the numerical results. Table 3.2 shows the effect of different initial time increment size on the time required to reach the steady state. The larger initial time increments tend to predict a higher time to reach the steady state as shown in the Table 3.2.

Table 3.2. Effect of different initial time increment size

\begin{tabular}{|c|c|}
\hline $\begin{array}{c}\text { Initial Time } \\
\text { Increment (seconds) }\end{array}$ & $\begin{array}{c}\text { Steady State } \\
\text { Time (hours) }\end{array}$ \\
\hline 4000 & 52.22 \\
2000 & 50.56 \\
1000 & 49.72 \\
500 & 49.17 \\
200 & 48.83 \\
100 & 48.72 \\
50 & 48.67 \\
10 & 48.66 \\
\hline
\end{tabular}

With the initial time increment size of 4000 seconds the steady state is reached at around 52.2 hours whereas with the initial time increment of 1000 seconds, the steady state is reached at 49.7 hours. A difference of around $5 \%$ in the steady state times is observed. For an initial time increment of 200 seconds the steady state is reached at 
even a lower value of time equal to 48.8 hours. The difference in the steady state time reduces to around $1.5 \%$ when compared with the initial time increment size of 1000 seconds. Thus with the smaller time increments the difference in the time required to reach the steady state decreases. It is observed that on reducing the time increment convergence in terms of steady state time is achieved. For the time increments less than 50 seconds negligible difference is observed at the steady state times. Further reduction in the time increment results into insignificant resolution at the cost of large computational time. As a result, it is concluded that an initial time increment can be chosen in the range: $10-50 \mathrm{~s}$ in order to obtain an accurate time required to reach the steady state to the moisture diffusion problem.

Another parametric study is done to determine appropriate tolerance on the moisture diffusion study. The value of this parameter indicates that if all the nodal moisture concentrations are changing at less than a certain rate (tolerance) then the FE analysis considers to have reached the steady state. Table 3.3 shows the effect of different tolerances on the time required to reach the steady state. With the tolerance of 1e-5 the calculated steady state to the moisture diffusion problem is obtained at around 12.2 hours as compared to the calculated steady state time of 126.6 hours with the tolerance of 1e-11. With the tolerance of $1 \mathrm{e}-11$ the moisture concentration at the time 126.6 hours throughout the body reaches a value of $1 \mathrm{gm} / \mathrm{mm}^{3}$, which indicates that the body has reached the actual steady state. Whereas with the tolerance of $1 \mathrm{e}-5$ the moisture concentration at time 12.2 hours is $0.7 \mathrm{gm} / \mathrm{mm}^{3}$ in the bottom skin of the sandwich beam, indicating that body has not reached the actual steady state although the 
FE analysis indicates the numerical steady state to have reached. For the tolerance of 1e-7 it is observed that moisture concentration throughout the sandwich beam is nearly 1 $\mathrm{gm} / \mathrm{mm}^{3}$ with the least moisture concentration $0.99722 \mathrm{gm} / \mathrm{mm}^{3}$ in the bottom layer of the sandwich beam. With this tolerance (1e-7) the steady state is reached at time 49.72 hours. Thus, even with the tolerance value of $1 \mathrm{e}-7$ moisture concentration throughout the sandwich structure is almost $1 \mathrm{gm} / \mathrm{mm}^{3}$. It is therefore concluded that a tolerance can be chosen in the range: $1 \mathrm{e}-7$ to $1 \mathrm{e}-11$ in order to represent moisture concentration profile throughout the sandwich composite. In the entire study further, a tolerance of $1 \mathrm{e}-$ 7 is chosen so as to reduce the computational time required while preserving the accuracy of the solution desired.

Table 3.3. Effect of different tolerance on the convergence rate

\begin{tabular}{|c|c|c|}
\hline Tolerance & $\begin{array}{c}\text { Steady State } \\
\text { Time (hours) }\end{array}$ & $\begin{array}{c}\text { Moisture } \\
\text { concentration } \\
\text { at the furthest } \\
\text { distance }\end{array}$ \\
\hline $1.00 \mathrm{E}-05$ & 12.22 & 0.717 \\
$1.00 \mathrm{E}-07$ & 49.72 & 0.997 \\
$1.00 \mathrm{E}-08$ & 68.33 & 0.9997 \\
$1.00 \mathrm{E}-09$ & 86.94 & 0.999972 \\
$1.00 \mathrm{E}-11$ & 117.12 & 1.0 \\
\hline
\end{tabular}




\subsection{ANALYSES OF THE DEFORMATION IN THE VISCOELASTIC SANDWICH COMPOSITE}

\subsubsection{ANALYTICAL SOLUTION}

The solution to the deformation of a viscoelastic sandwich structure subjected to three point bending test can be obtained using the elasticity or to some extent strength of material approach. Here the strength of material approach has been used. The total deflection of the beam would comprise of bending and the shear components. For a sandwich beam with the span length $L$, width $b$, face and core thickness of $f$ and $c$, respectively and Young's moduli of the face and core to be $E_{f}$ and $E_{c}$ respectively, the bending component of deflection at the midspan of the beam $(L / 2)$ is written as:

$\delta_{b}=\frac{P L^{3}}{48(E I)_{e q}}$

Where, the equivalent flexural rigidity $(E I)_{e q}$ is written as:

$(E I)_{e q}=\frac{E_{f} b f c^{2}}{2}$

Similarly, the shear deflection is written as:

$\delta_{s}=\frac{P L}{4\left(A G_{c}\right)}$

Here, $A$ cross sectional area and $G_{c}$ is the shear modulus of the core

Thus, the total deflection is written as: 
$\delta=\frac{P L^{3}}{48(E I)_{e q}}+\frac{P L}{4\left(A G_{c}\right)}$

The sandwich under consideration in this study consists of a linear viscoelastic foam core and linear elastic facings. Considering the time dependent behavior of the foam core, shear component of the total deflection increases with time due to the decrease in the shear modulus. The effect of the decreasing value of the material modulus of the viscoelastic core has negligible effect on the equivalent flexural rigidity. Therefore, the bending component is assumed to remain constant with time. Thus, the total time dependent deflection of the beam as shown by Gibson [30] is then written as:

$\delta(t)=\frac{P L^{3}}{48(E I)_{e q}}+\frac{P L J_{c}(t)}{4(A)}$

Here, $J_{c}(t)$ is the shear creep compliance of the foam core

Here $(E I)_{\mathrm{eq}}$ is assumed to be constant since the since the degrading modulus of the core has a negligible impact on the overall value of $(\mathrm{EI})_{\mathrm{eq}}$. The FE results obtained are verified with the analytical solution developed in Eq. (3.10). To accomplish this, a $254 \mathrm{~mm}$ long and $25.4 \mathrm{~mm}$ wide sandwich beam composed of $1 \mathrm{~mm}$ thick aluminum facings and $25.4 \mathrm{~mm}$ thick polyurethane foam core is subjected to three point bending test with point load of $250 \mathrm{~N}$ applied at the half span of the beam. The details of the loading and the boundary conditions are shown in the Fig 3.4. The calibrated timedependent parameters (Prony series) representing the creep compliance upto 1200 hours and elastic properties for the polyurethane foam core are given in Table 3.4. Similarly, 
the elastic properties of aluminum are given in Table 3.5. With these properties and testing conditions, the viscoelastic deformation at the midspan of the sandwich structure with two different mesh densities is as shown in Fig 3.5. A uniform mesh (relatively coarser) is used in case of FEA model 1 whereas a finer mesh in the region near the point of application of the load and the constrains is used in FEA model 2. As expected the response is captured more realistically in FEA model2 as compared to FEA model 1 as the localized effect of point load and point constrains is captured much better using a finer mesh near the point of application of the load and the constrains. In general, FE results are found to be in a good agreement with the analytical solution.

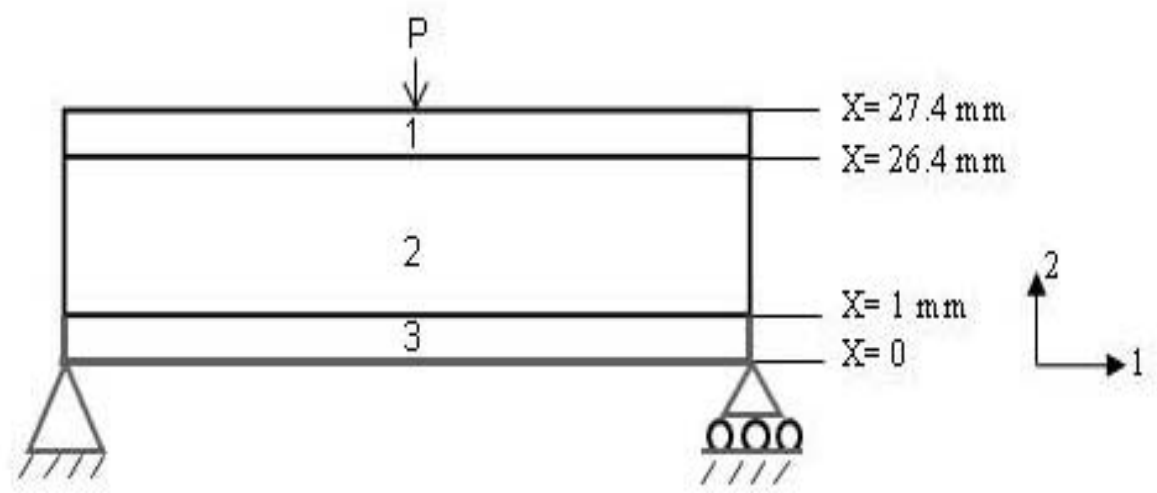

Fig. 3.4. Loading and boundary conditions on the sandwich beam 


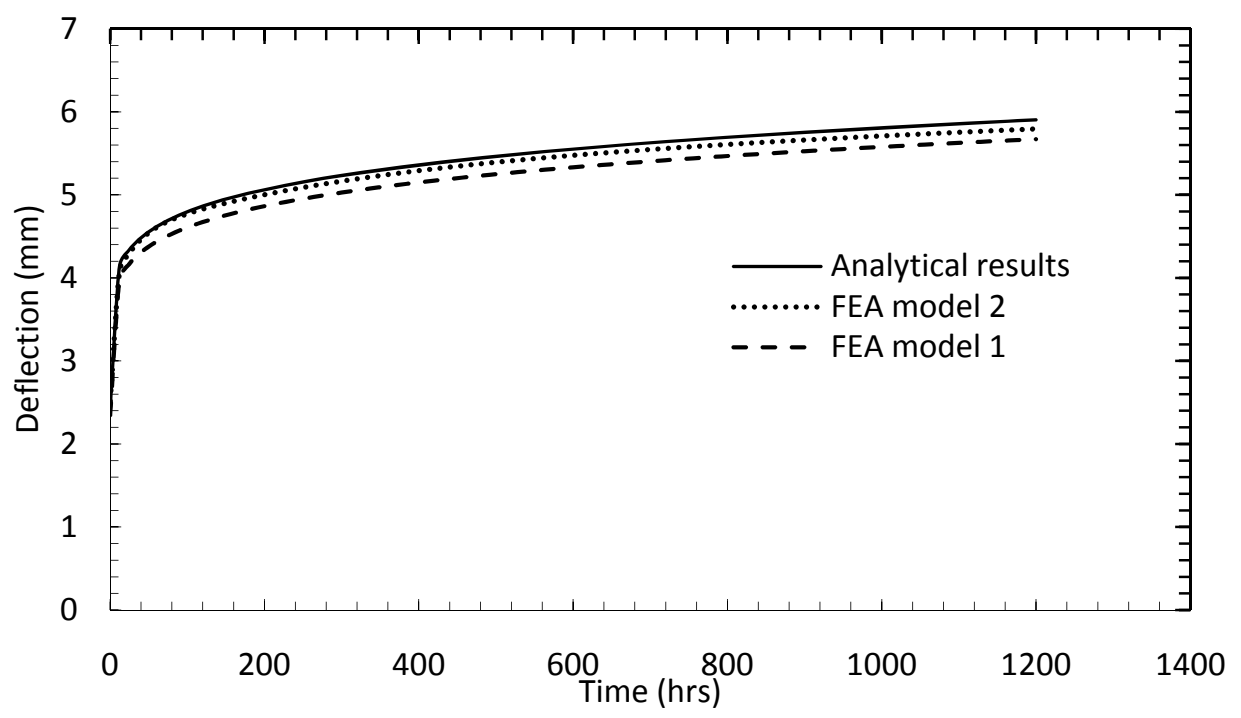

Fig. 3.5. Viscoelastic deformation at the midspan

Table 3.4. Elastic properties and Prony series coefficients for polyurethane foam core

\begin{tabular}{|c|c|c|}
\hline $\mathrm{n}$ & $\lambda_{n}\left(\mathrm{sec}^{-1}\right)$ & $D_{n} \times 10^{-3}\left(\mathrm{MPa}^{-1}\right)$ \\
\hline 1 & 1 & 2 \\
3 & $10^{-1}$ & 2.5 \\
4 & $10^{-2}$ & 4.25 \\
5 & $10^{-3}$ & 8.4 \\
6 & $10^{-4}$ & 10.68 \\
7 & $10^{-5}$ & 11.45 \\
8 & $10^{-6}$ & 24 \\
\hline & $10^{-7}$ & \\
\hline & $\mathrm{E}=31 \mathrm{Mpa}$ & \\
\hline
\end{tabular}


Table 3.5. Elastic properties of aluminum facings

\begin{tabular}{|c|c|}
\hline $\mathrm{E}(\mathrm{MPa})$ & $v$ \\
\hline 70,000 & 0.29 \\
\hline
\end{tabular}

\subsubsection{CONVERGENCE STUDY USING FE METHOD FOR DEFORMATION}

When FE approach is used to obtain various field variables such as displacement, stresses and strains for the sandwich beam subjected to a three point bending test, it is extremely essential to check the adequacy of the type of element and the size of elements used. In order to validate the adequacy of the size of elements used in the FE mesh, a sandwich beam composed of carbon-epoxy facings and divinycell $\mathrm{H}-250$ core is subjected to a three point bending test with a load of $2018 \mathrm{~N}$ at the center of the beam. Table 3.6 shows the elastic properties of carbon epoxy faces and the divinycell H-250 core. It is assumed that the facings and the core are linear elastic materials. The problem is a modeled using the two dimensional continuum plane strain elements (CPE4) in ABAQUS. The total deflection at the midspan of the beam obtained is shown in Fig 3.6. The results from FE code are in good agreement with the experimental data. This ensures the adequacy of the number of elements used in the mesh. 


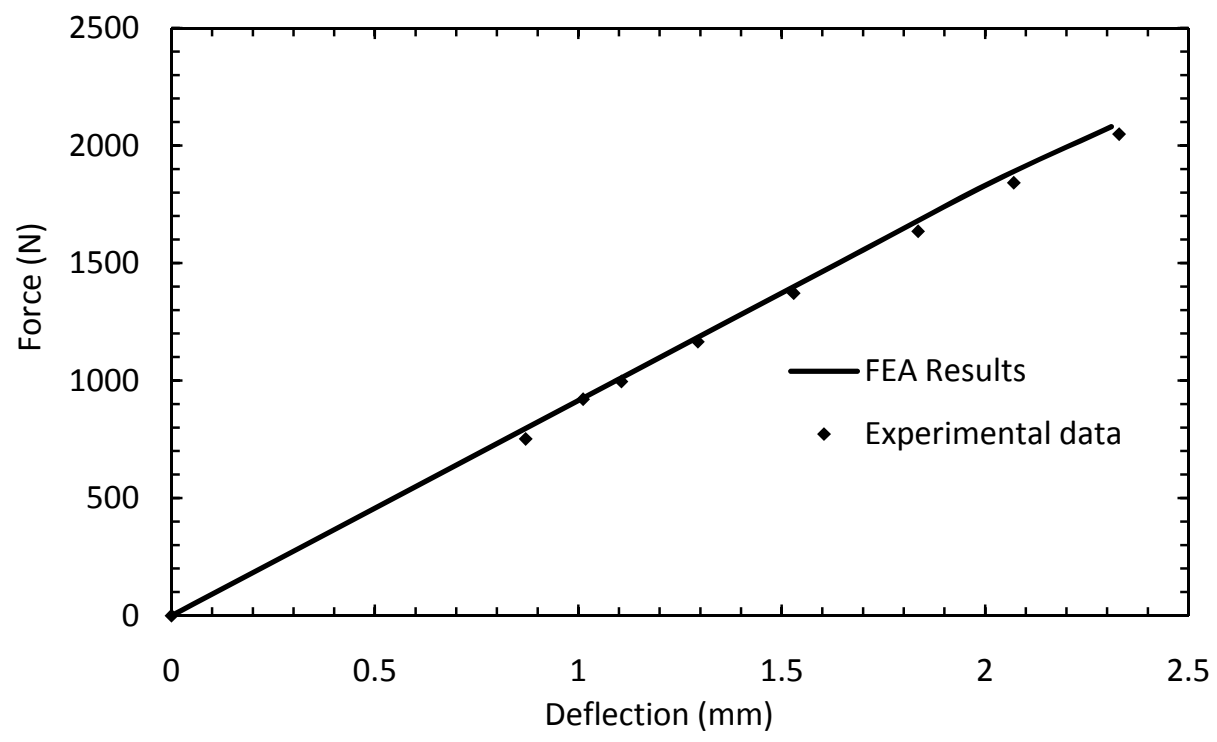

Fig. 3.6. Deflection at the mid span of the sandwich beam 
Table 3.6. Elastic properties of Carbon Epoxy laminate and Divinycell H250

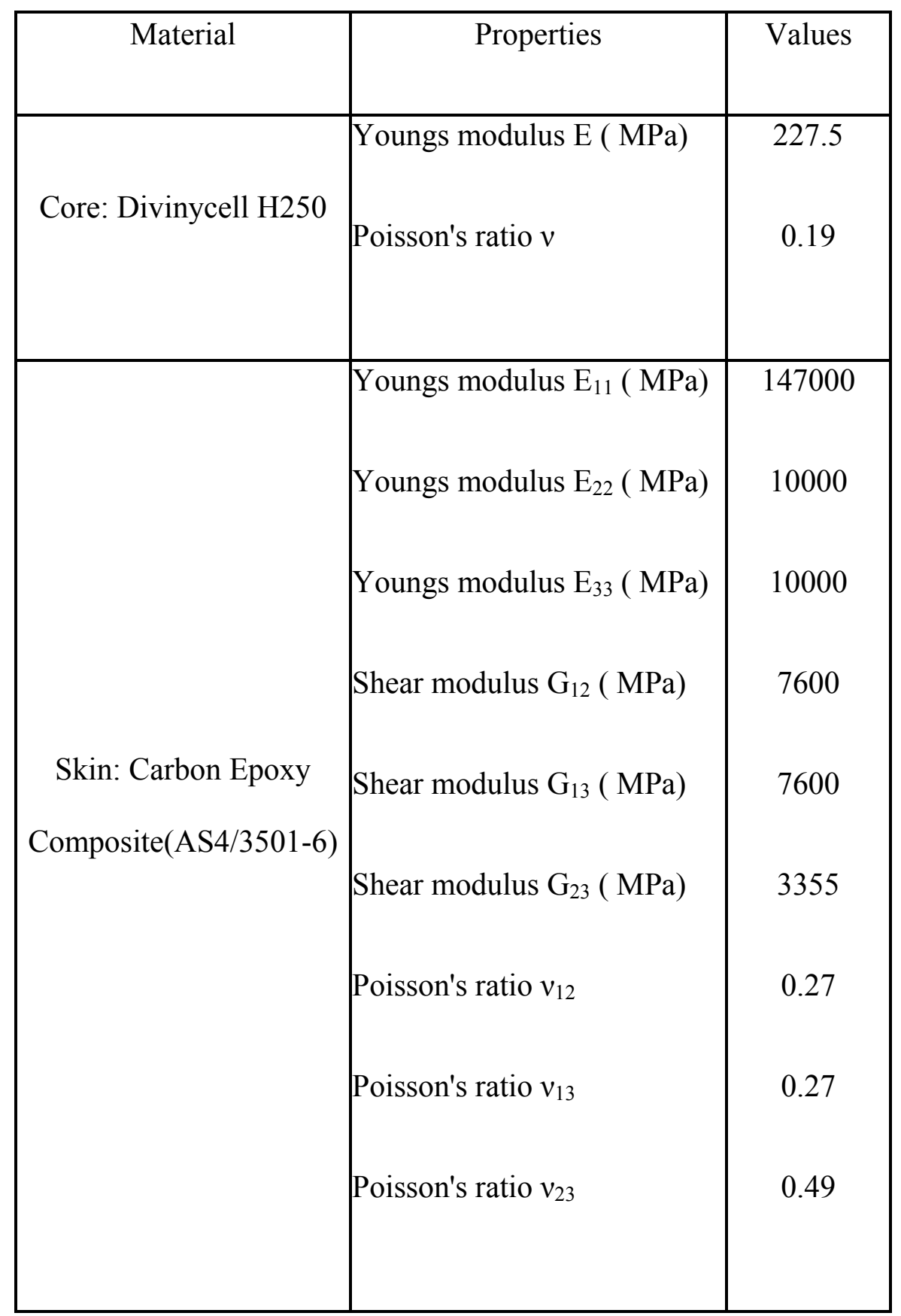

For sandwich beam with the viscoelastic core, the response to the applied static (creep) load is time dependent and hence the size of the initial time increment 
significantly affects the instantaneous (elastic) material response. A parametric study is performed to examine the effect of different initial time increment sizes on the instantaneous material response. This study helps to determine the range of initial time increment that can be used to simulate creep testing of the sandwich beam using FE code. A sandwich beam composed of orthotropic carbon epoxy facing and polyurethane foam core is subjected to a three point bending test with a load of $250 \mathrm{~N}$ applied at the center of the beam. The instantaneous creep displacement values for different time increment sizes at the midspan of the beam on the bottom surface are shown in the Fig 3.7. It is very clear the initial time increment size can significantly affect the accuracy of the results. Large time increments can lead to a diverging solution. As a result, it is concluded that an initial time increment can be chosen in the range: $0.001-0.01 \mathrm{~s}$ in order to represent an instantaneous response of this sandwich beam. 


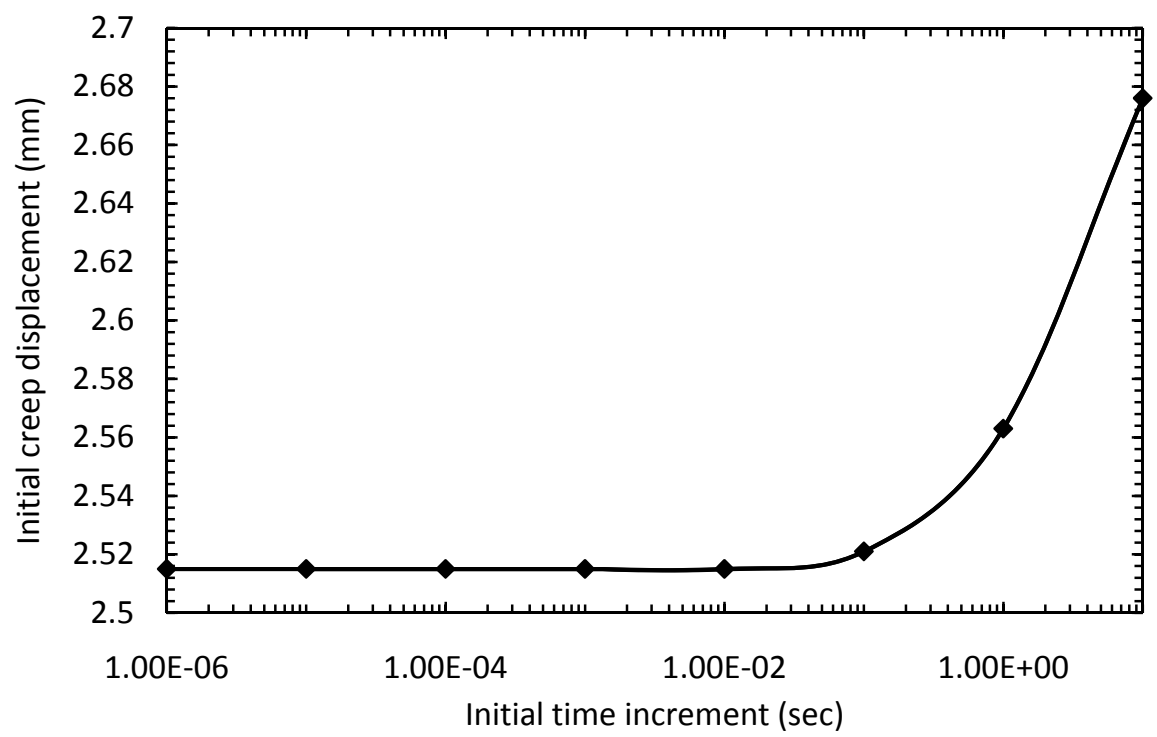

Fig. 3.7. Effect of different time increments sizes on instantaneous displacements

\subsection{COUPLED MOISTURE DIFFUSION AND DEFORMATION IN THE}

\section{VISCOELASTIC SANDWICH BEAM}

In this study, a sandwich beam subjected to simultaneous moisture diffusion and mechanical loading is considered. The sandwich beam composed of orthotropic linear elastic carbon-epoxy laminated skin and isotropic viscoelastic polyurethane foam core is subjected to simultaneous moisture prescribed at the surface of the top skin and a point load at the midspan of the beam in a three point bending test. The solution to the coupled displacement-transport equations is obtained in two sequential steps. In the first step, the moisture diffusion profile through the sandwich beam is obtained. Once the moisture distribution throughout the sandwich beam during the transient analysis is known, the deformation of the viscoelastic sandwich composite with moisture dependent 
material properties are determined. It must also be noticed that the analysis does not address a fully coupled problem. While the deformation depends on the moisture concentration, the moisture concentration can be obtained without the knowledge of stress/strain. The moisture diffusion process is not stress or strain assisted. It is assumed that material properties (material modulus and Poisson's ratio) of the carbon epoxy facings with $0^{\circ}$ fiber orientation are independent of the moisture concentration. Because for the $0^{\circ}$ fiber orientation the effect of moisture on the material properties is negligible [5]. It is assumed that the instantaneous part $\left(\mathrm{E}_{0}\right)$ of the polyurethane foam core degrades linearly with moisture concentration as per Eq. (3.11).

$E_{0}=\frac{1}{D_{0}}=E_{0}-\alpha * \frac{c}{C_{0}}$

where $C_{0}\left(\mathrm{gm} / \mathrm{mm}^{3)}\right.$ is the applied moisture concentration, $\alpha(\mathrm{MPa})$ is the slope of the graph of instantaneous modulus $\mathrm{E}_{0}(\mathrm{MPa})$ versus nondimensionalized moisture concentration $c / C_{0}$.

The parameter $\mathrm{g}_{0}$ that measures the moisture dependent elastic compliance can therefore be written as:

$$
g_{0}=\frac{E_{0}}{\left(E_{0}-\alpha * \frac{c}{C_{0}}\right)}
$$

The specific choice of the type of degradation is made to merely illustrate that the strain induced due to the degradation can be pronounced. The magnitude of the strain certainly depends upon the form of degradation assumed. For more severe 
degradation than the linear form, the magnitude of strains could be much higher. In this case the value of $\alpha$ is chosen to be $12 \mathrm{MPa}$. The Eq. (3.12) is then written as:

$$
g_{0}=\frac{E_{0}}{\left(E_{0}-12 * \frac{c}{C_{0}}\right)}
$$

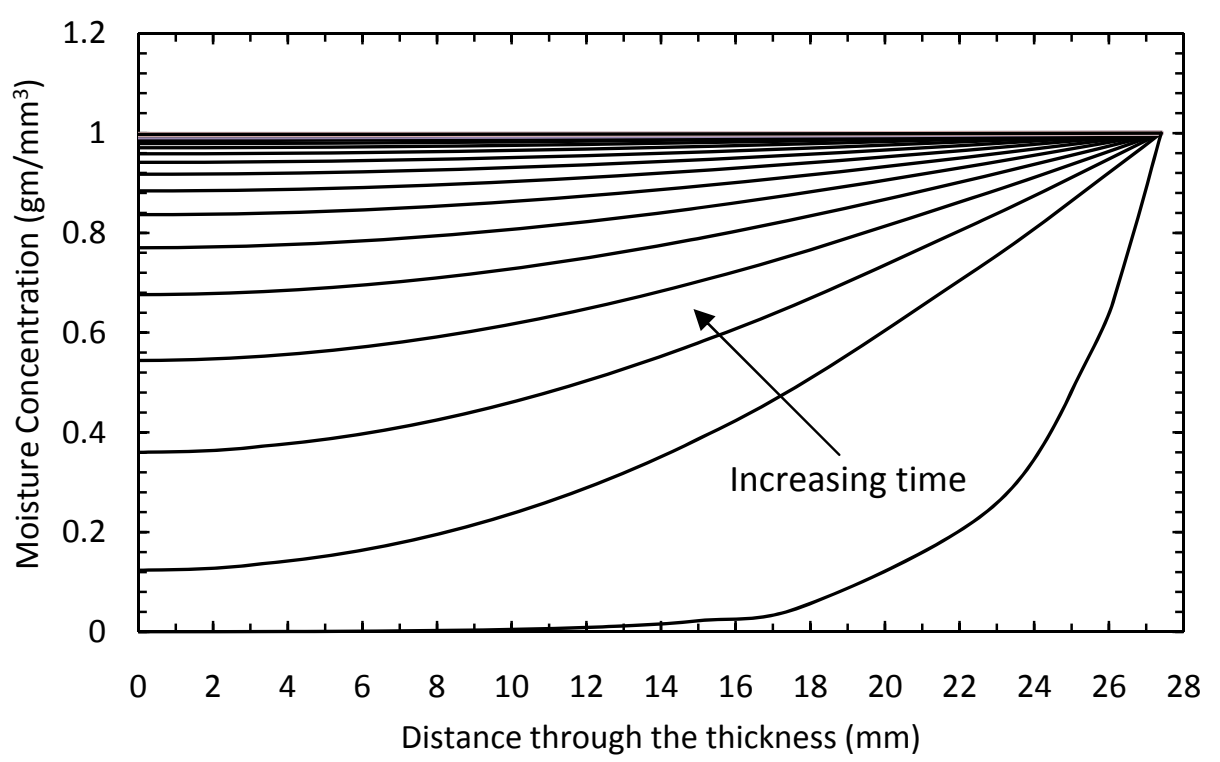

Fig. 3.8. Evolution of concentration of diffusing moisture 


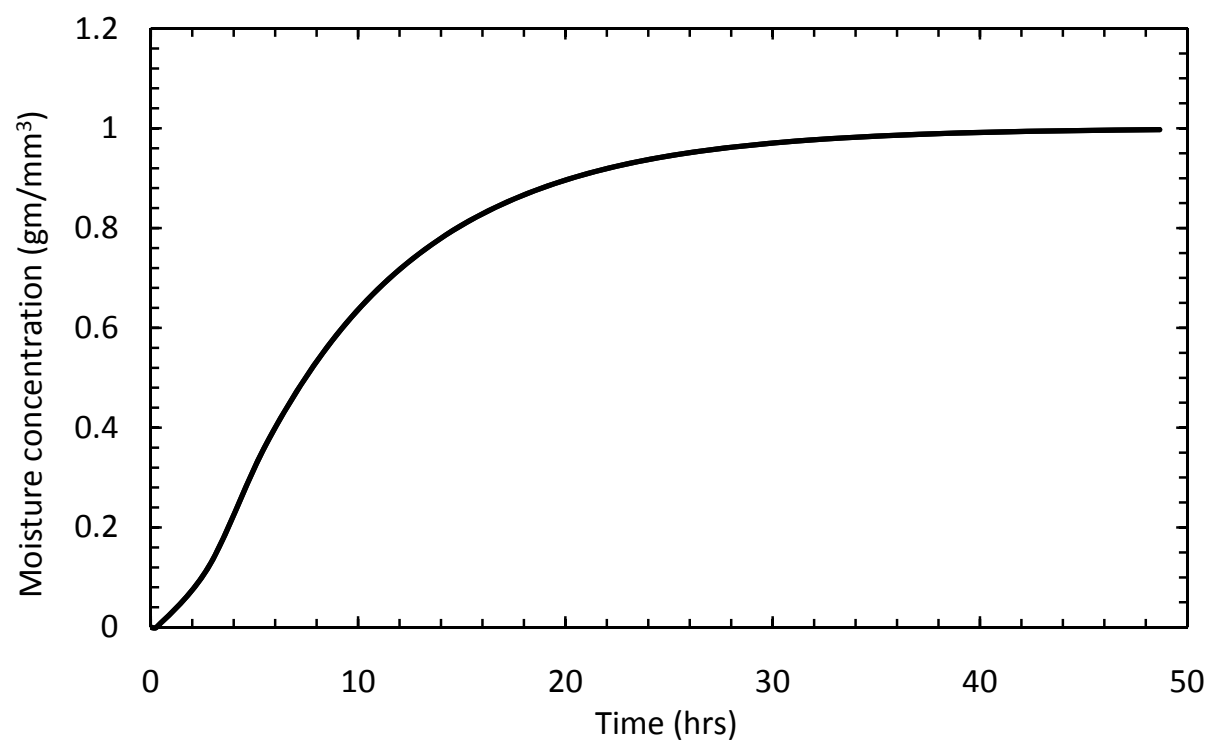

Fig. 3.9. Evolution of concentration of diffusing moisture at a fixed location of $x=0$

The evolution of the concentration of moisture is plotted in Fig 3.8. The moisture diffusivities of the carbon-epoxy facings is assumed to be $1.06 \mathrm{E}-03 \mathrm{~mm}^{2} / \mathrm{sec}$ and that of the polyurethane core equal to $1.06 \mathrm{E}-02 \mathrm{~mm}^{2} / \mathrm{sec}[31]$. Thus, the ratio of the moisture diffusivity of the skin to that of the core materials is 0.1 . With the moisture diffusion governed by the one dimensional (through the thickness) Fick's law of diffusion, the boundary and initial conditions as per the Eqs. (3.5) and (3.6) and the concentration applied at the surface of the top skin, the sandwich composite reaches a steady state when the moisture concentration at the bottom skin reaches a value of the applied moisture concentration of $1 \mathrm{gm} / \mathrm{mm}^{3}$. Fig 3.9 shows the evolution of the moisture diffusion profile at a fixed distance $(\mathrm{x}=0)$ in the bottom skin. 
The degradation of the instantaneous modulus $\left(\mathrm{E}_{0}\right)$ due to the moisture concentration is incorporated in the constitutive material model in the Eq. (2.3) and is solved numerically and implemented in the material subroutine (UMAT) of the ABAQUS FE code. 2D (CPE4) and 3D (C3D8) solid continuum elements are used to solve the coupled problem of moisture diffusion and deformation in the sandwich beam. The results obtained using 3D elements are in good agreement with those obtained using 2D elements. Fig 3.10 shows the graph of transverse displacement through the thickness of the beam at the midspan at $\mathrm{t}=1200$ hours using both $2 \mathrm{D}$ and $3 \mathrm{D}$ elements. In order to reduce the computational time required the further analysis is performed using $2 \mathrm{D}$ elements. The analysis is performed upto 1200 hours (greater than the steady state time of 432 hours for moisture diffusion). To highlight the effect of moisture degradation on the overall response of the sandwich beam, three cases are considered. 


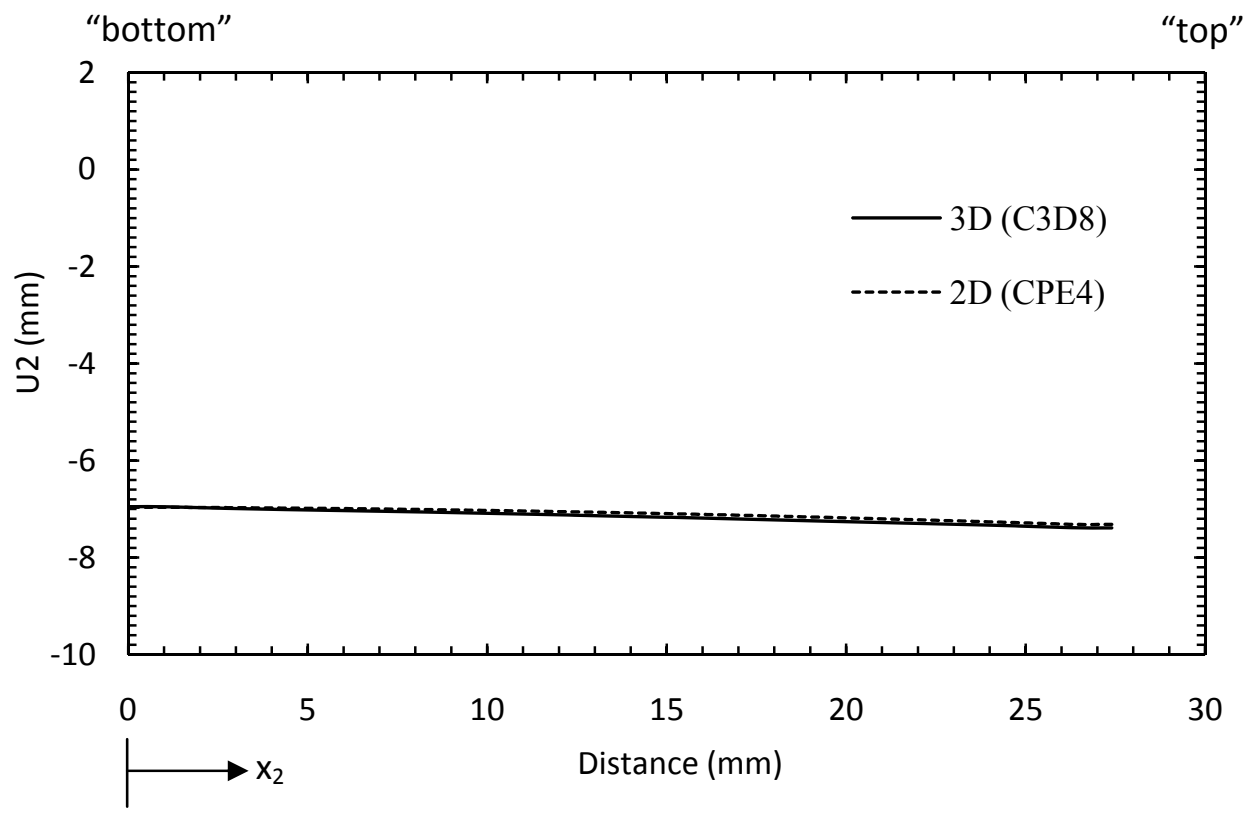

Fig. 3.10. Comparison of transverse displacement using 2D and 3D elements at 1200 hours

case (i) The sandwich beam composed of orthotropic elastic carbon epoxy laminated skin and linear elastic polyurethane foam core whose material modulus degrades with the moisture concentration as governed by the Eq. (3.11).

case (ii) The sandwich beam composed of orthotropic elastic carbon epoxy laminated skin and isotropic linear viscoelastic polyurethane foam core without any effect of moisture concentration.

case (iii) The sandwich beam composed of orthotropic elastic carbon epoxy laminated skin and isotropic linear viscoelastic polyurethane foam core with the instantaneous material modulus degrading with the moisture concentration as governed by the Eq. (3.11). 
The effect of degradation on the displacement in the transverse direction $\left(\mathrm{U}_{2}\right.$, direction of loading) at the midspan of the beam is as shown in the Fig 3.11. At a distance of half the thickness of the sandwich beam $(x=13.5 \mathrm{~mm})$ an increase of about $23 \%$ in the transverse displacement is observed in the case (iii) as compared to case (ii) at time $\mathrm{t}=1200$ hours. This is because of the decreasing instantaneous modulus of the viscoelastic polyurethane foam core with time. There is an increase of about $46 \%$ in the transverse displacement in the case (iii) as compared to the case (i). This shows that the effect of moisture degradation is found to be more pronounced in case of the viscoelastic materials because of their time dependent behavior. At time $\mathrm{t}=0$ the displacements in all the three cases is the same as expected.

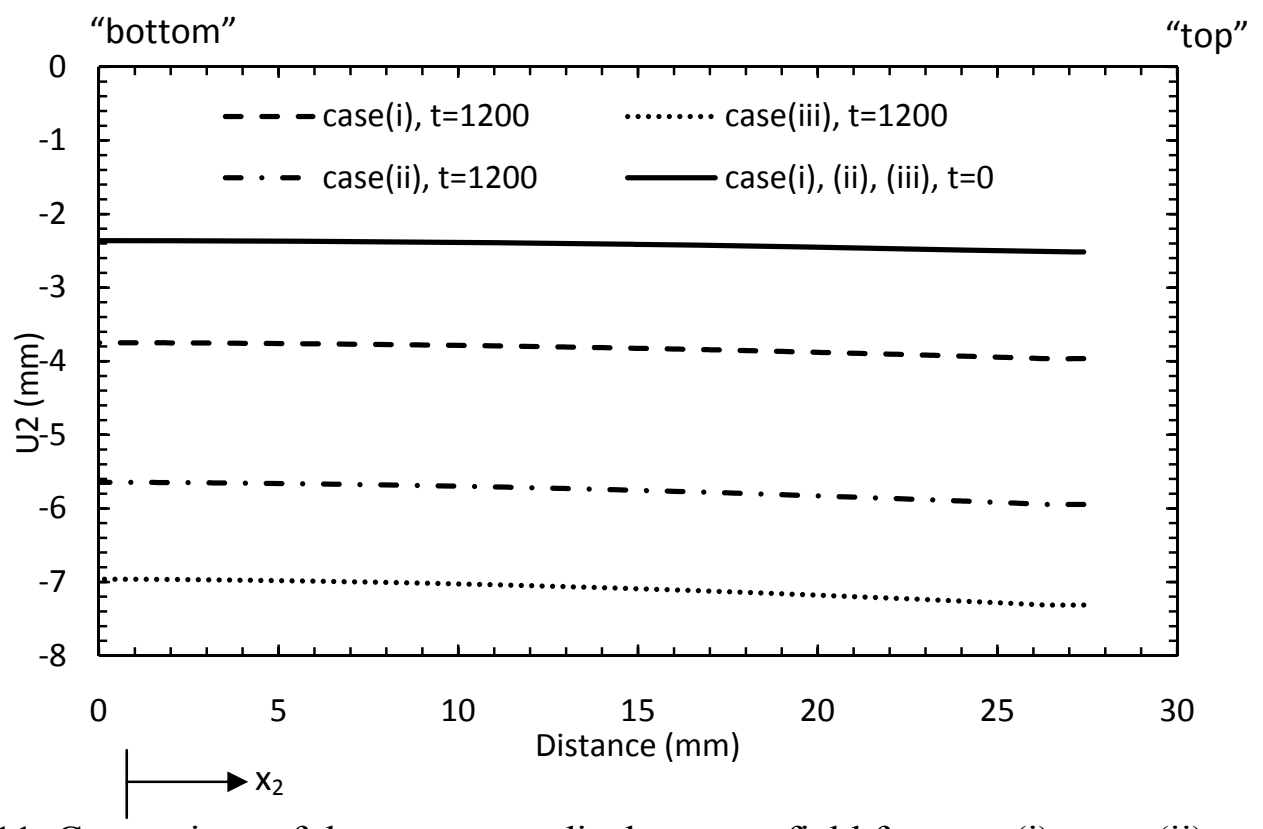

Fig. 3.11. Comparison of the transverse displacement field for case (i), case (ii) and case 
The effect of degradation on the transverse strain $\left(\varepsilon_{22}\right)$ at the midspan of the beam is shown in Fig 3.12. A jump in the transverse strain is observed at both the top and the bottom interfaces of the sandwich beam. The increase in the transverse strain in the top skin at the interface $(\mathrm{x}=26.4 \mathrm{~mm})$ for the case (iii) is $8 \%$ more as compared to the case (ii). Whereas an increase of $13.3 \%$ in the degrading core for the case (iii) is observed as against the case (ii) at the interface ( $x=26.4 \mathrm{~mm})$. The magnitude of the jump in case (iii) is the maximum and at the top interface is about $13.5 \%$ higher as compared to the case (ii) at time $t=1200$ hours. Whereas the magnitude of jump at the bottom interface is about $22 \%$ higher as compared to the case (ii) at time $\mathrm{t}=1200$ hours. It is worth noting that for the jump exceeding a certain critical value the transverse strain can aid to the delamination at the interface. An increase of approximately $30 \%$ in the top skin at the interface for the case (iii) is observed as compared to the case (i). Whereas the transverse strain in the degrading core at the interface $(\mathrm{x}=26.4 \mathrm{~mm})$ for the case (iii) is as high as $52 \%$ when compared to the case (i). The magnitude of the jump in case (iii) at the top interface is about $35 \%$ higher as compared to the case (i) at time $t=1200$ hours. Whereas the magnitude of jump at the bottom interface is about $52 \%$ higher as compared to the case (ii) at time $\mathrm{t}=1200$ hours. This shows that the effect of moisture degradation on the behavior of viscoelastic materials is much more pronounced as compared to the elastic materials. 


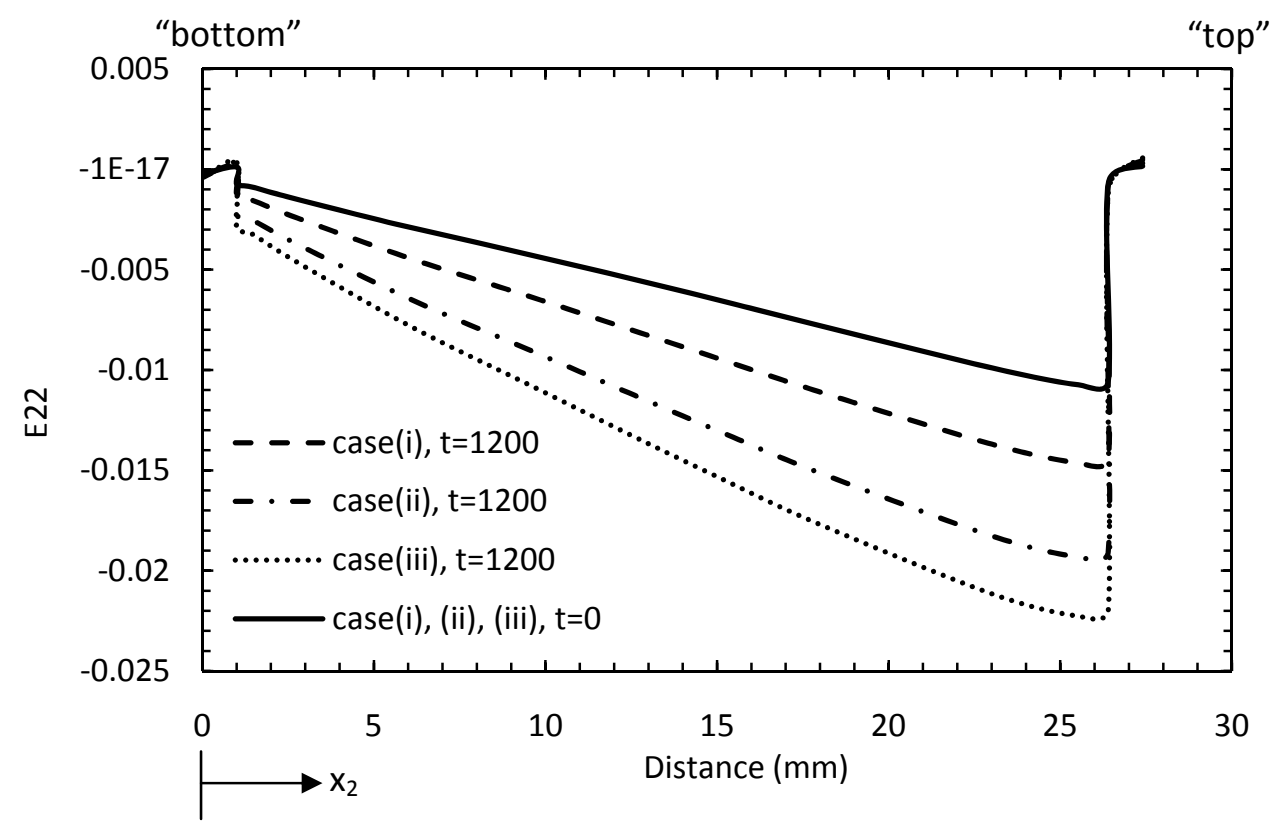

Fig. 3.12. Comparison of the transverse strain field for case (i), case (ii) and case (iii)

The Fig 3.13 shows the effect of moisture degradation on the longitudinal displacement $\left(\mathrm{U}_{1}\right)$ at the midspan of the beam. The longitudinal displacement in case (iii) is about $28 \%$ higher in comparison to the case (ii) at time $t=1200$ hours. An increase of about $100 \%$ in the displacement is observed in case (iii) as compared to case (i). 


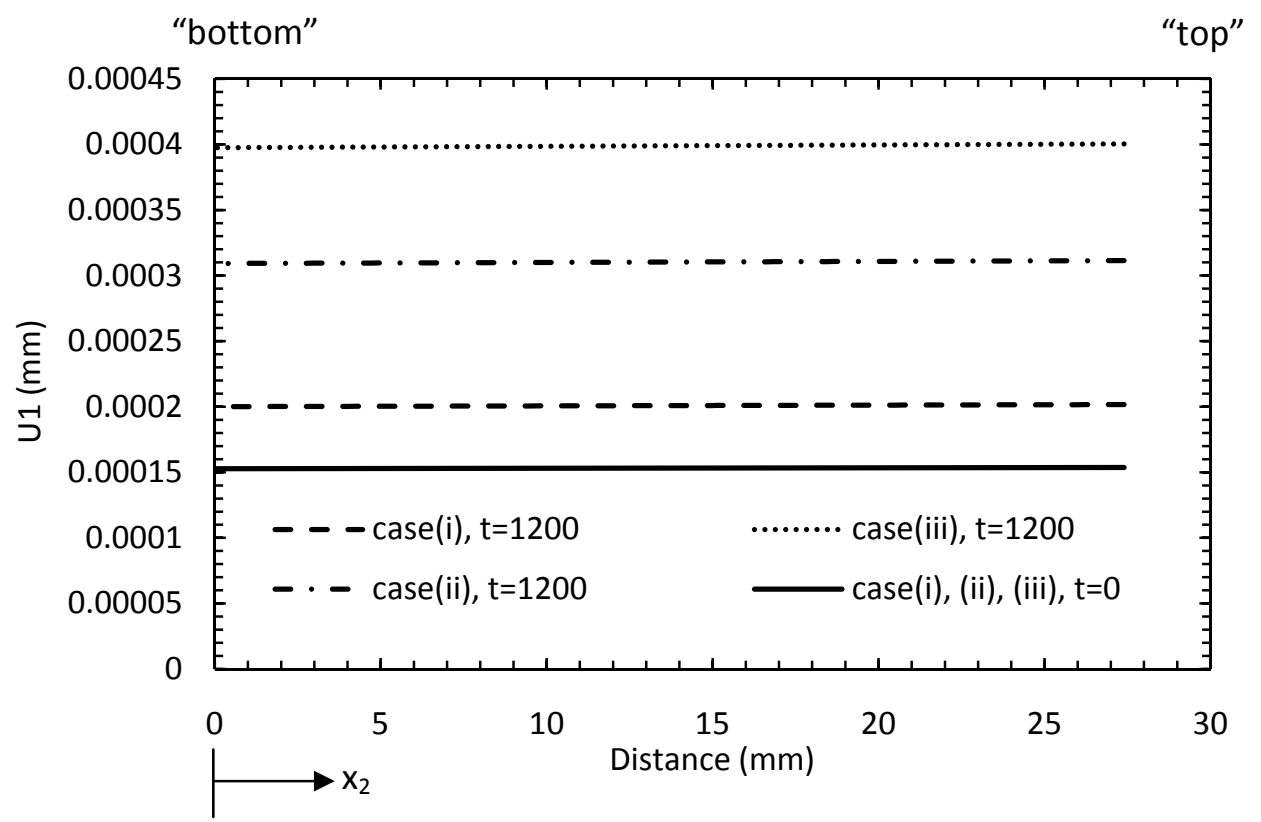

Fig. 3.13. Comparison of the longitudinal displacement field for case (i), case (ii) and case (iii)

Fig 3.14 shows the effect of moisture degradation on the longitudinal strain $\left(\varepsilon_{11}\right)$ at the midspan of the beam. As expected the longitudinal strain is maximum for the case (iii) as compared case (ii) and case (i). It is worth observing that there is no jump in the longitudinal strain at the interfaces, which satisfies displacement compatibility. 


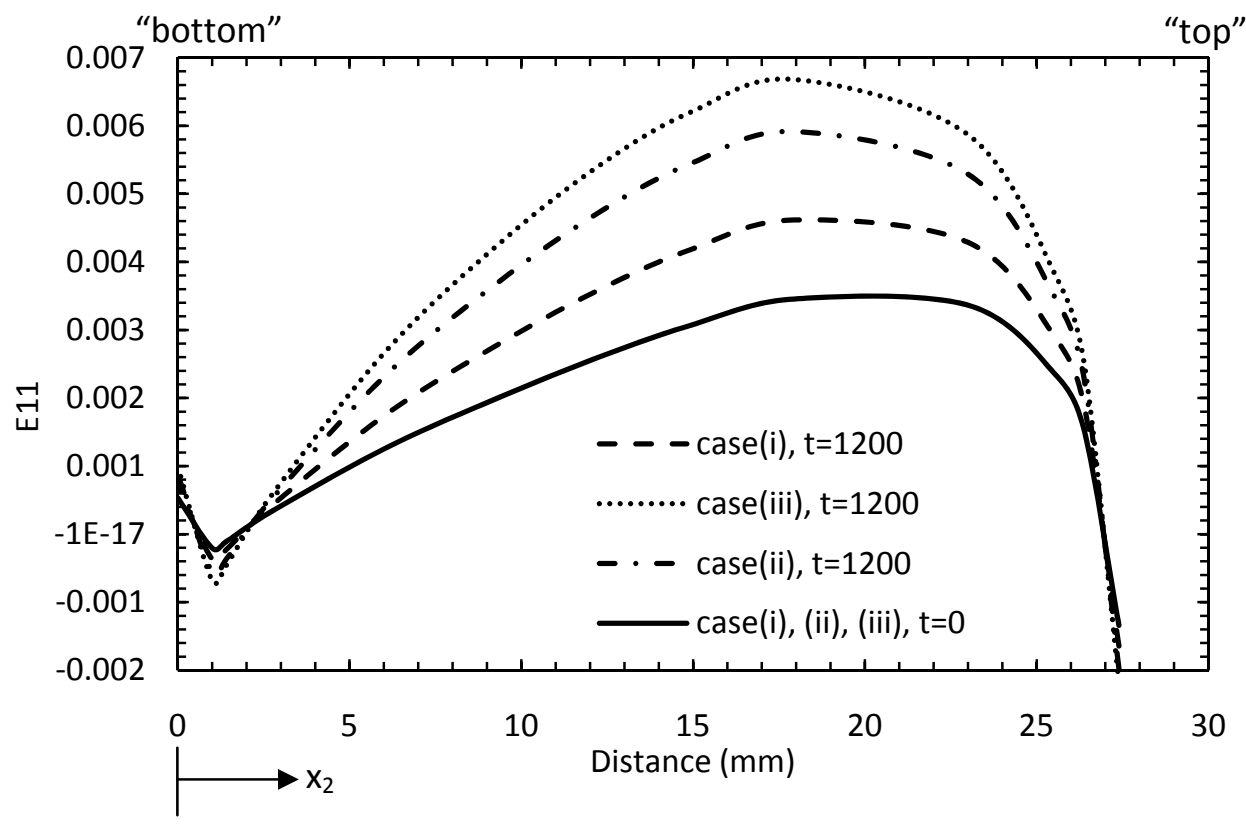

Fig. 3.14. Comparison of the longitudinal strain field for case (i), case (ii) and case (iii)

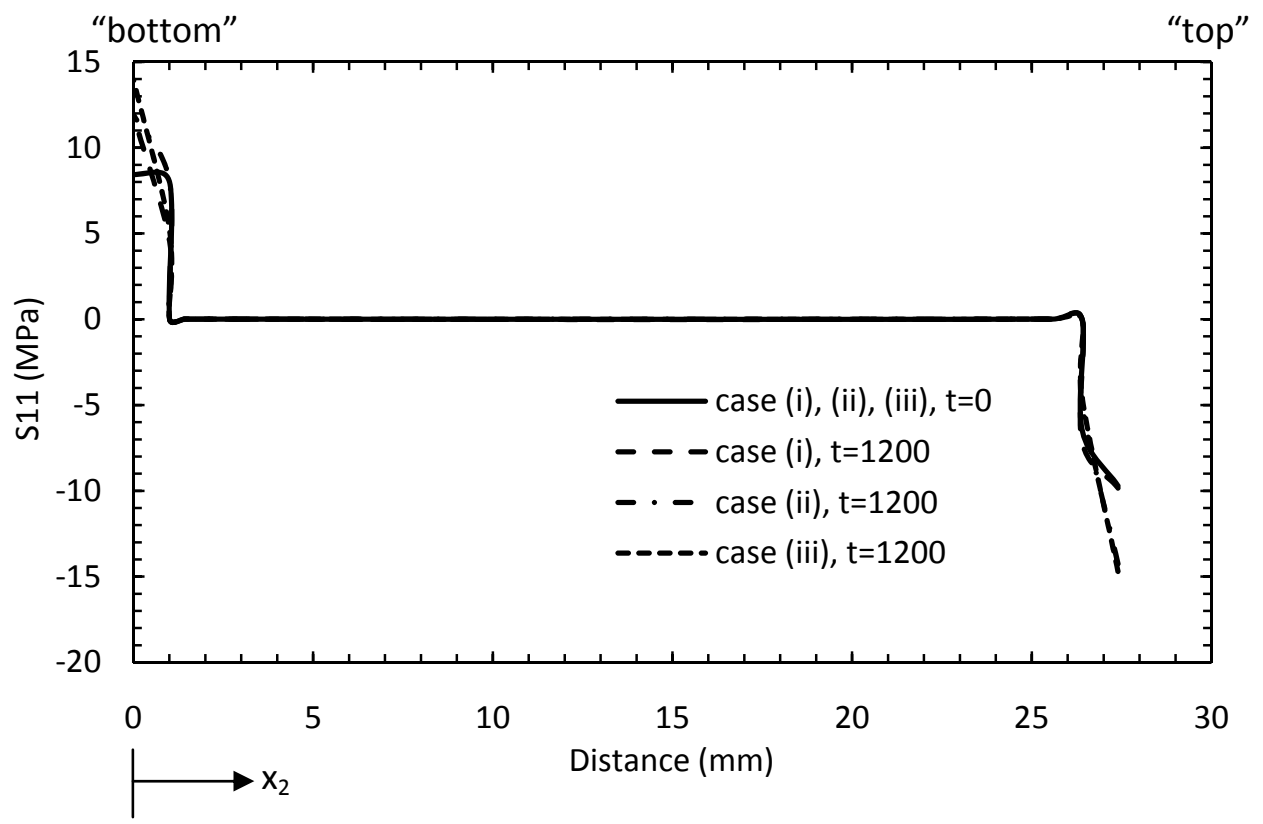

Fig. 3.15. Comparison of the bending stress field for case (i), case (ii) and case (iii) 
The effect of moisture degradation on the longitudinal (bending) stress $\left(\sigma_{11}\right)$ at a distance of $70 \mathrm{~mm}$ from the midspan of the beam is shown in the Fig 3.15. At the midspan (point of application of load) highly localized stress concentrations are observed as expected which will be discussed in more detail in the later part of this study. As we move away from the point of application of load the effect of localized stress concentration goes on diminishing. Thus, in order to avoid the effect of localized stress concentrations the stresses are measured at a distance of $70 \mathrm{~mm}$ from the midspan of the beam. The stress distribution throughout the beam can been seen from the contour plots presented in the later part of the discussion in this section. In all the three cases, the bending stresses at time $\mathrm{t}=1200$ hours are higher than the stresses at initial time $\mathrm{t}=0$ hours as expected. The magnitude of the bending stresses for all the times greater than $\mathrm{t}=0$ hours is maximum in case (iii) as compared to the case (ii) and case (i). The magnitude bending stresses at all the times greater than $t=0$ hours in case (ii) is higher in comparison to case (i) highlighting the effect of the time dependent behavior of the viscoelastic materials in comparison to the elastic materials. A jump in the bending stresses at the top and the bottom interfaces in the sandwich beam is also observed. The magnitude of the jump in case (iii) is the maximum and at the top interface is about $9.5 \%$ higher as compared to the case (ii) at time $t=1200$ hours. Whereas the magnitude of jump at the bottom interface is about $17 \%$ higher as compared to the case (ii) at time $\mathrm{t}=1200$ hours. This jump can act as a critical parameter in deciding the delamination at the interface. It is worth observing that even though the increase in the jump at the bottom interface is higher but the magnitude of the jump is higher at the top interface. 
Fig 3.16 shows the effect of moisture on the shear strain $\left(\varepsilon_{12}\right)$ at a distance of 70 $\mathrm{mm}$ from the midspan of the beam. As expected the shear strains at time $\mathrm{t}=0$ for all the three cases is the same. Shear strains in the core (through the thickness) are almost constant in all the three cases at a particular time and increases with increase in time. At time $t=1200$ the shear strains are maximum in case (iii) as compared to case (i) and case (ii). In other words, the shear strain at time $\mathrm{t}=1200 \mathrm{hrs}$ in case (i) is $48 \%$ lower as compared to case (iii) and in case (ii) is $20 \%$ lower as compared to case (iii).

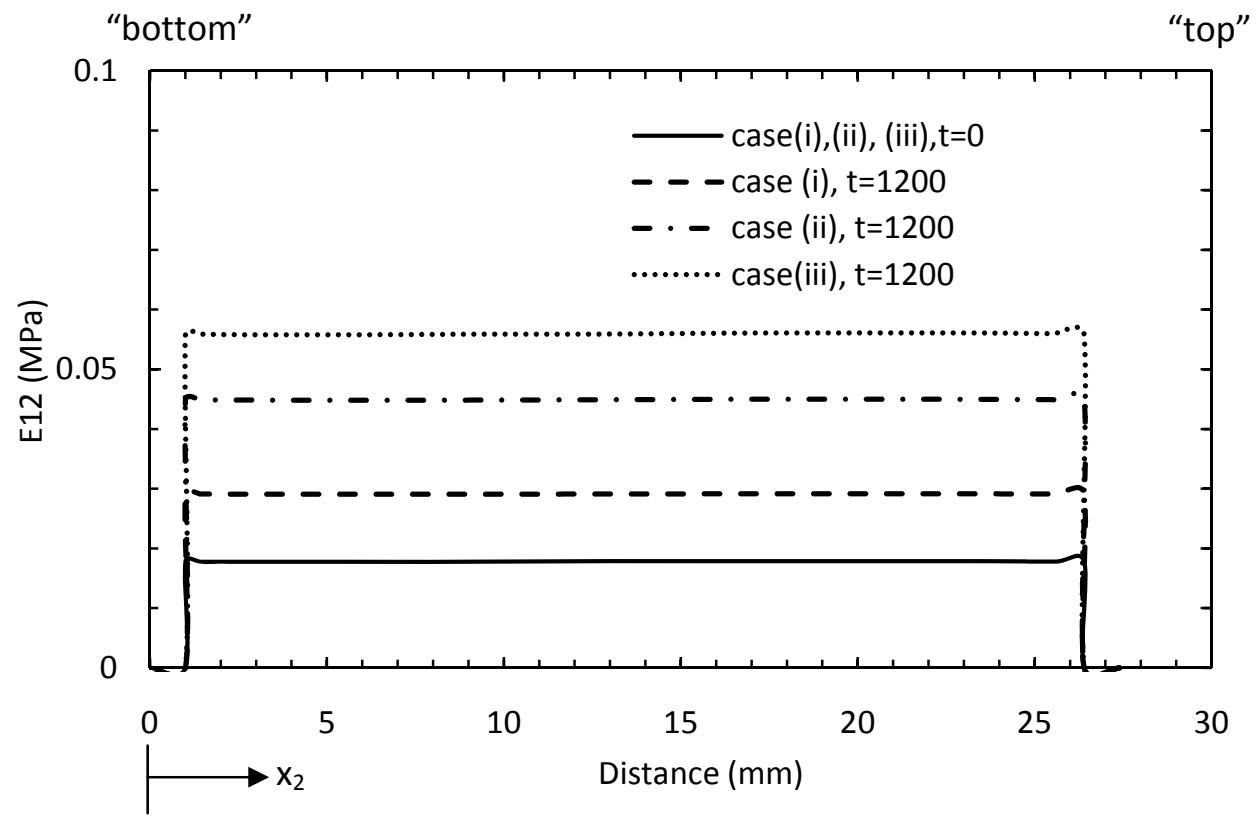

Fig. 3.16. Comparison of the shear strain field for case (i), case (ii) and case (iii)

Fig 3.17 shows the effect of moisture on the shear stress $\left(\sigma_{12}\right)$ at a distance of 70 $\mathrm{mm}$ from the midspan of the beam. As expected high shear stresses are observed in the core as compared to the skin in all the three cases at every time. In addition to it, the 
shear stresses in the core are nearly constant throughout the thickness. A jump of around $0.02 \mathrm{MPa}$ in the shear stresses at the top and the bottom interfaces in all the three cases is observed at time $\mathrm{t}=0$. The change in the jump with time is negligible in all the three cases.

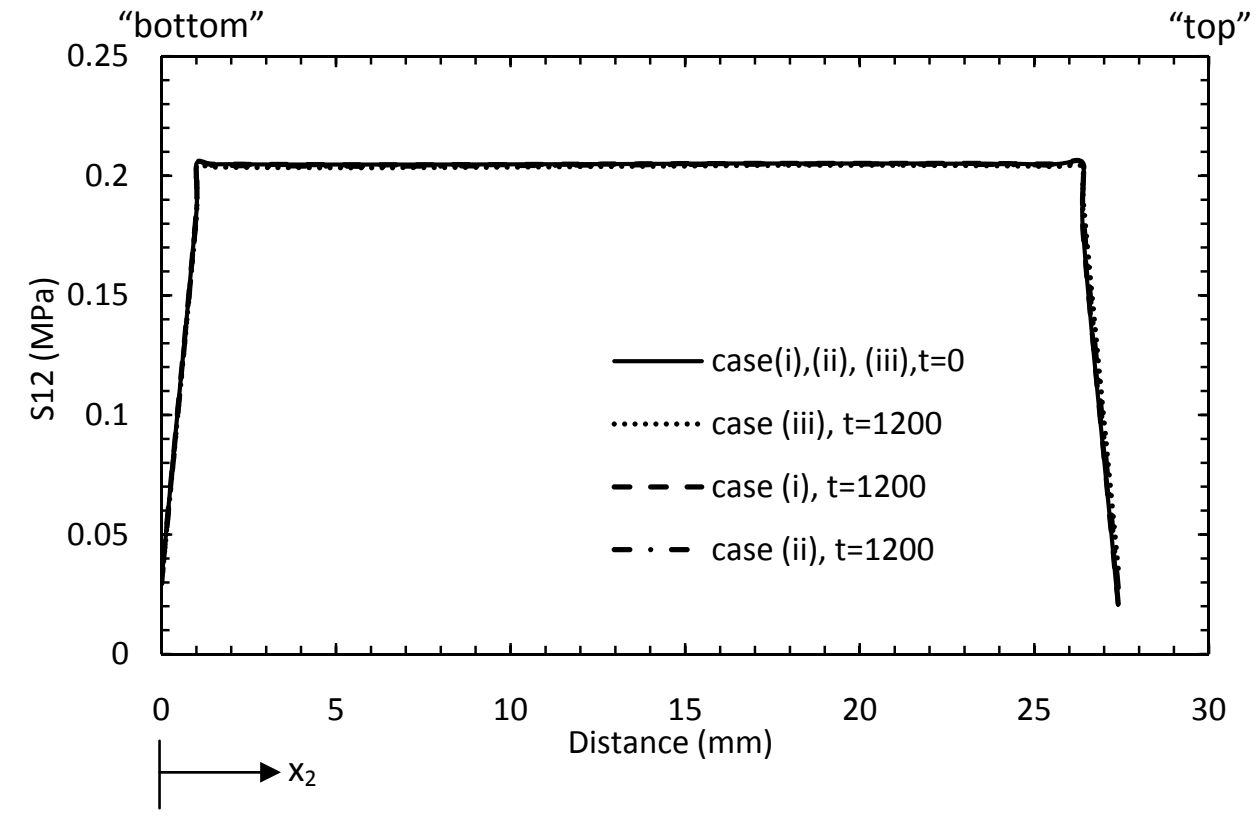

Fig. 3.17. Comparison of the shear stress field for case (i), case (ii) and case (iii)

The contour plots at time $\mathrm{t}=1200$ hours of the stress, strain and displacement fields are shown in Figs 3.18 to 3.25. In the region near the point of application of the load and the region near the constraints localized stress concentration effect is observed as expected. The stresses are therefore measured at a distance of $70 \mathrm{~mm}$ from the midspan of the beam where the effect is almost negligible. 


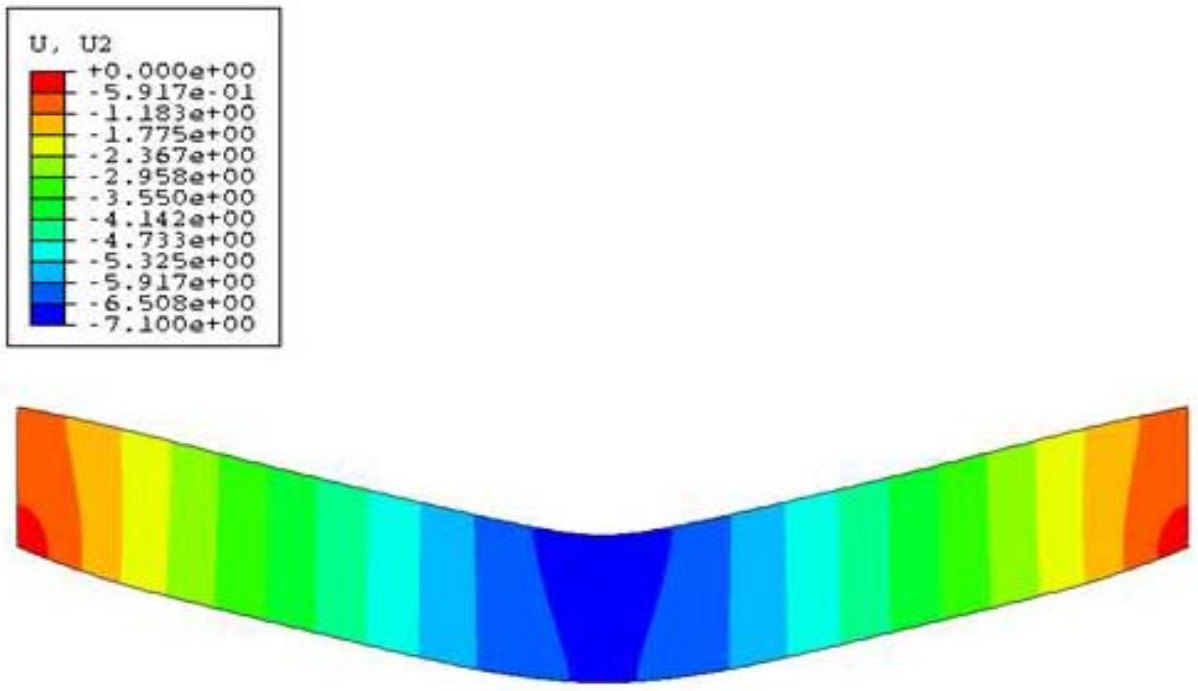

Fig. 3.18. Contour plot of transverse displacement $\left(\mathrm{U}_{2}\right)$ at 1200 hours

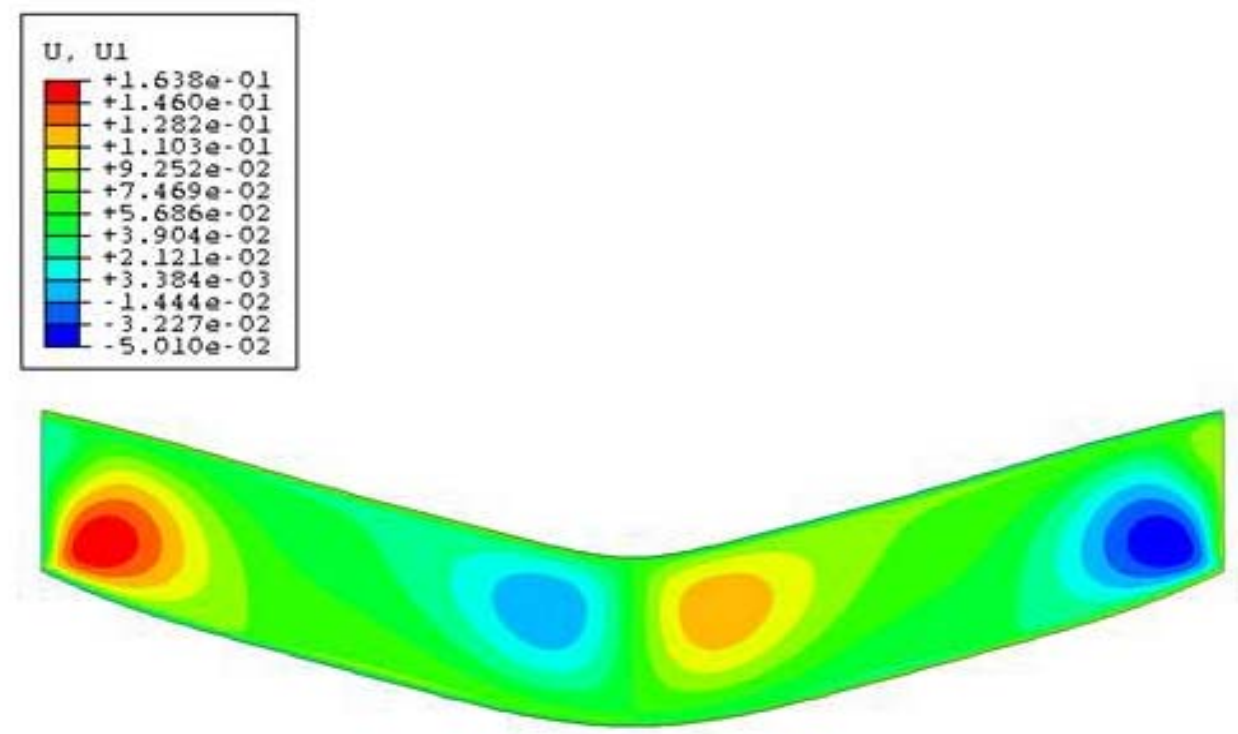

Fig. 3.19. Contour plot of axial displacement $\left(U_{1}\right)$ at 1200 hours 

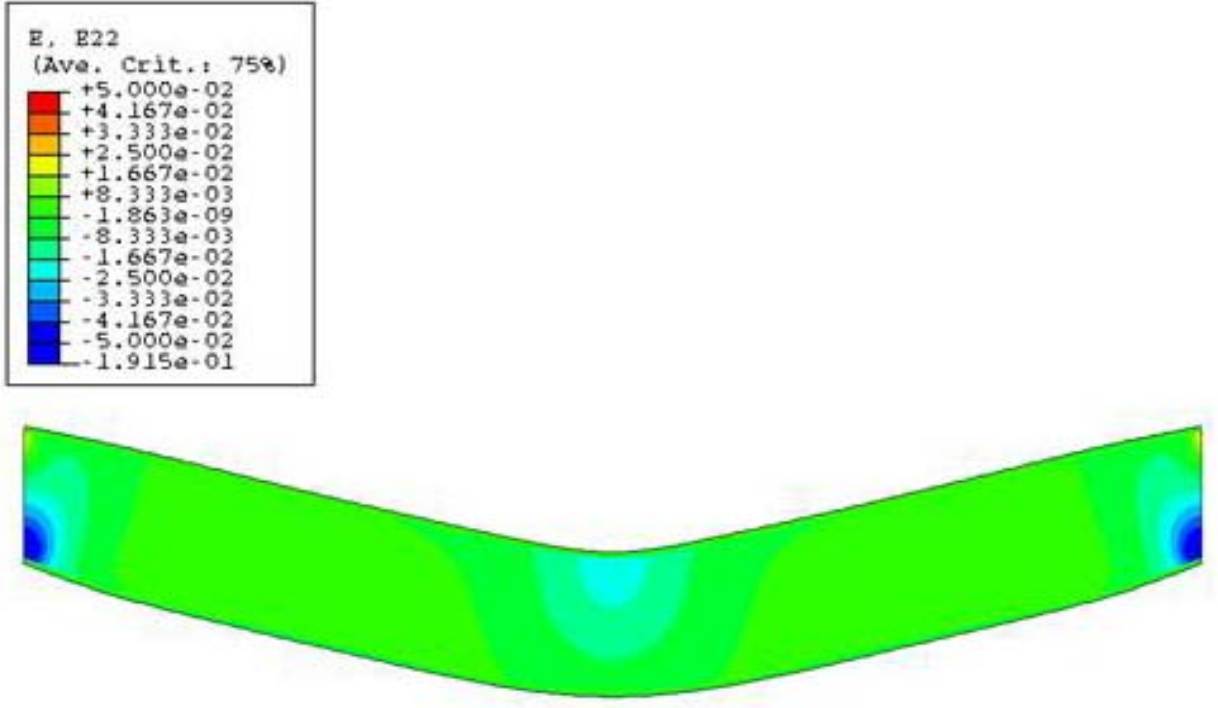

Fig. 3.20. Contour plot of transverse strain $\left(E_{22}\right)$ at 1200 hours

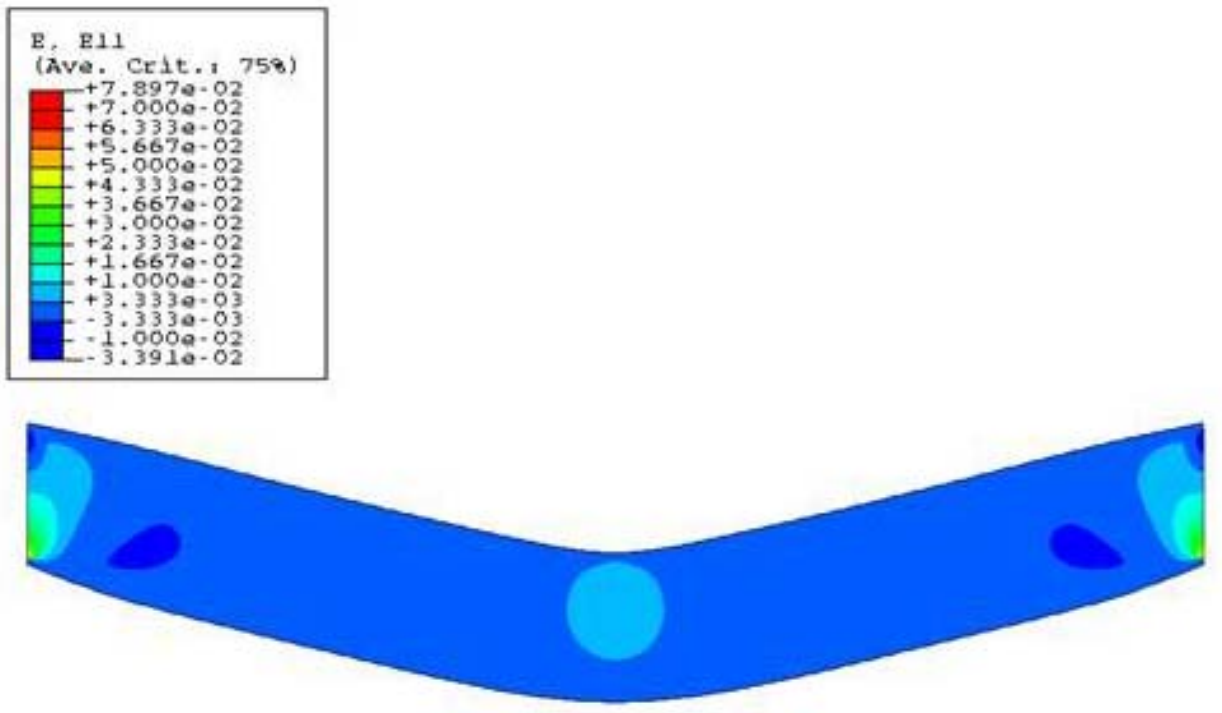

Fig. 3.21. Contour plot of axial strain $\left(E_{11}\right)$ at 1200 hours 

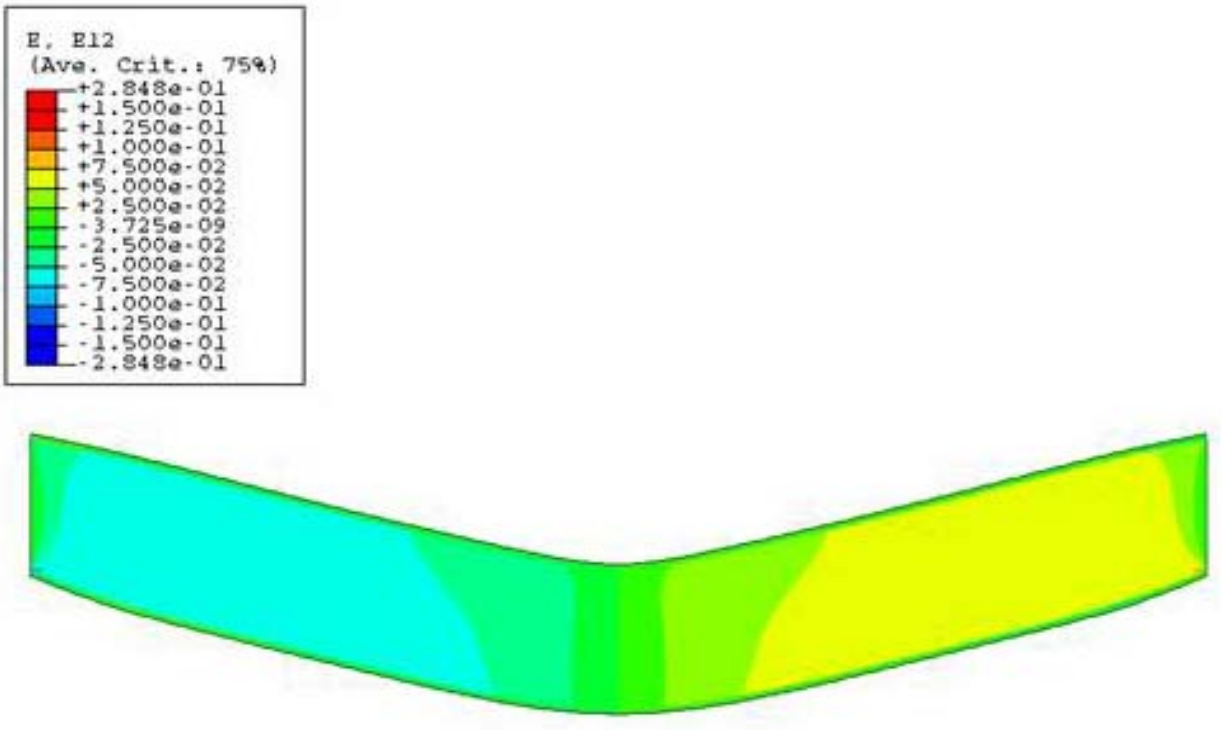

Fig. 3.22. Contour plot of shear strain $\left(E_{12}\right)$ at 1200 hours
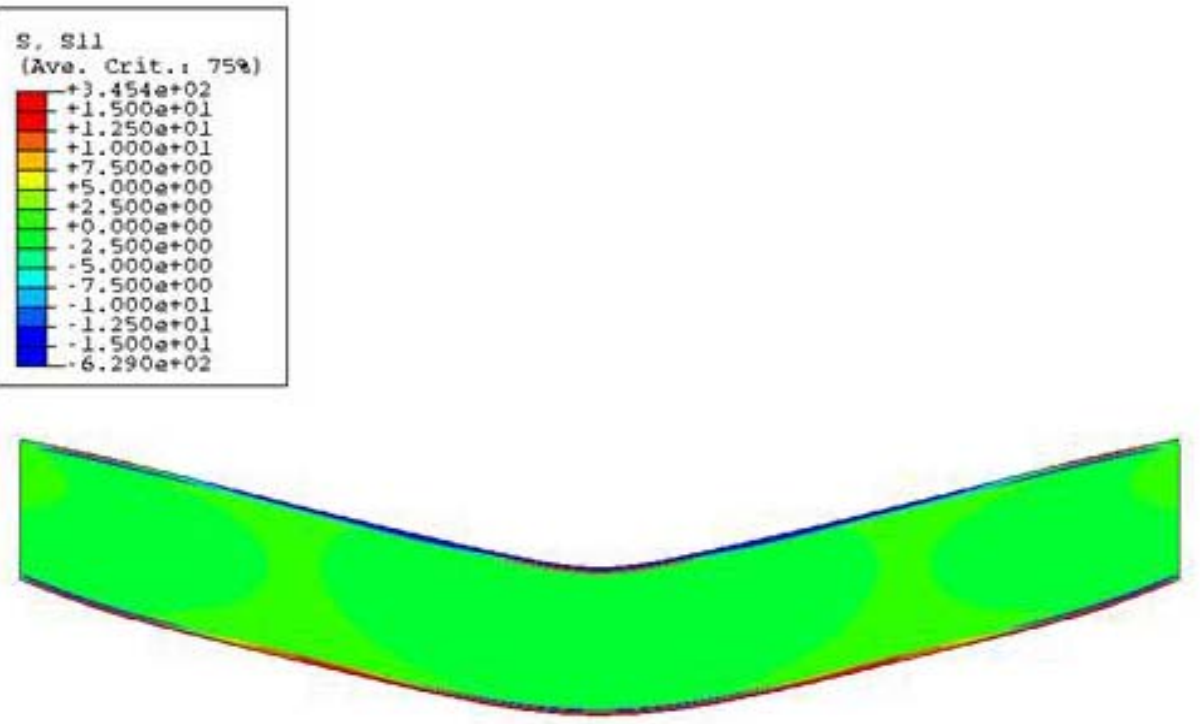

Fig. 3.23. Contour plot of bending stress $\left(\sigma_{11}\right)$ at 1200 hours 

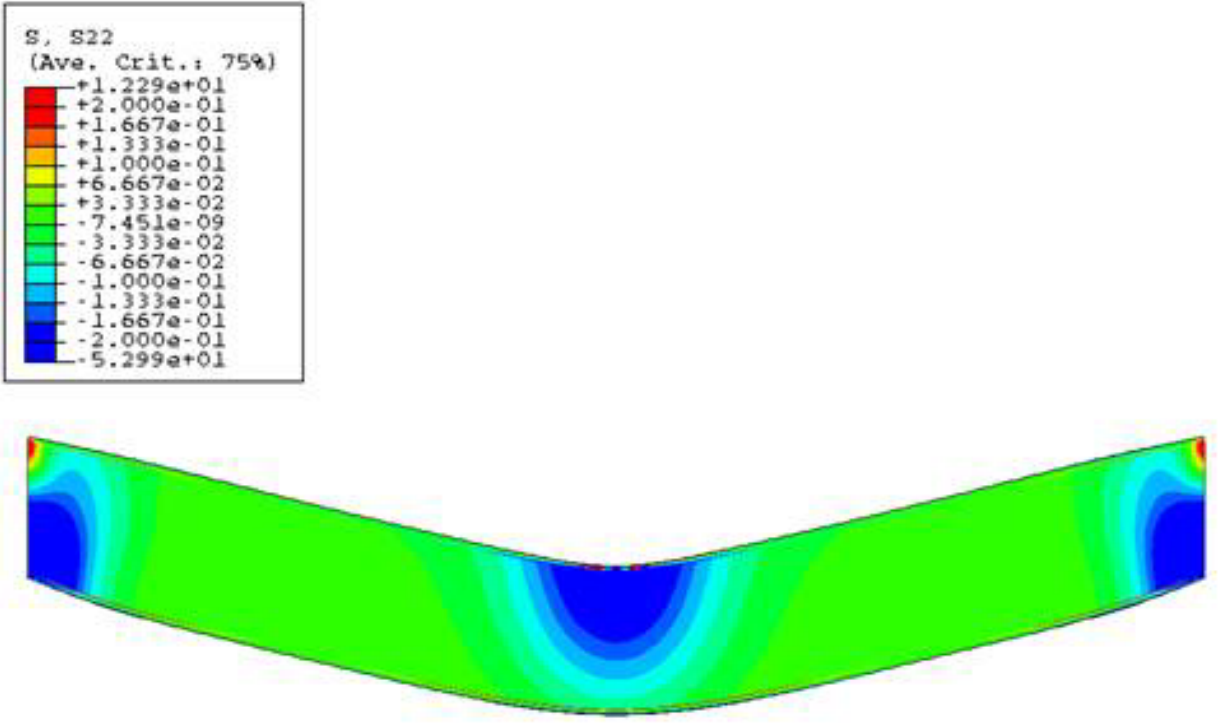

Fig. 3.24. Contour plot of transverse stress $\left(\sigma_{22}\right)$ at 1200 hours

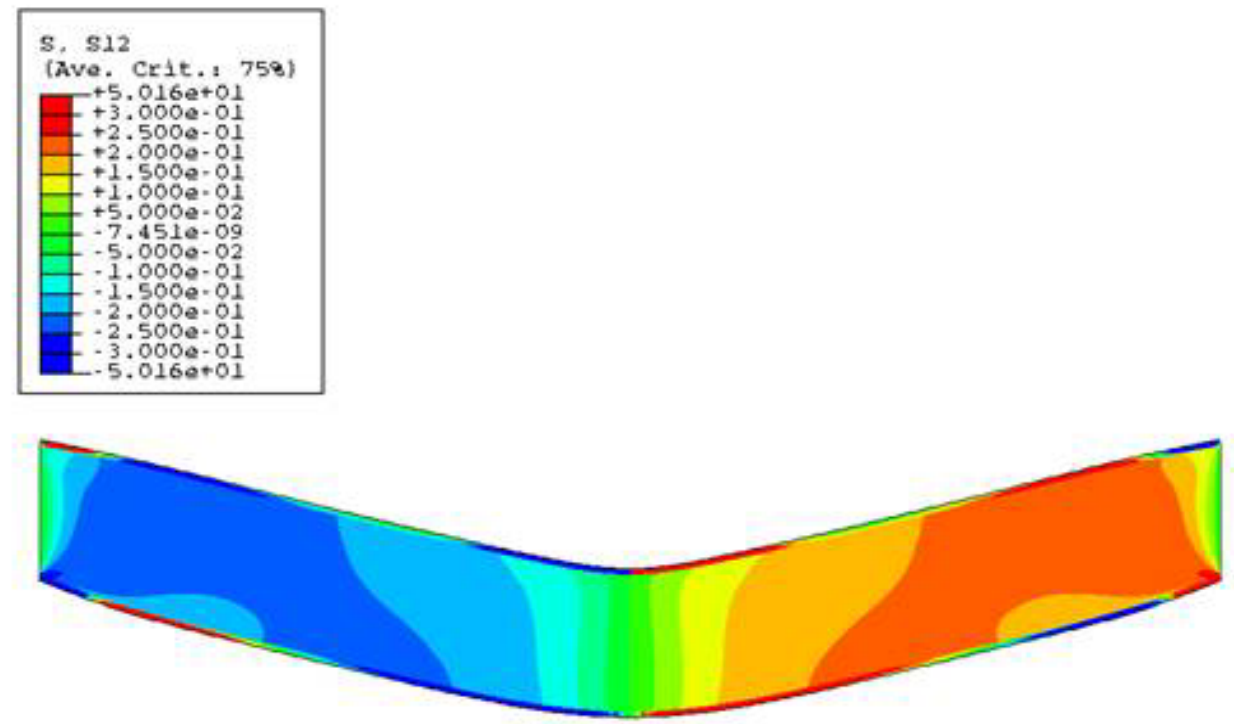

Fig. 3.25. Contour plot of shear stress $\left(\sigma_{12}\right)$ at 1200 hours 


\subsection{PARAMETRIC STUDY}

\subsubsection{EFFECT OF DIFFERENT MOISTURE DIFFUSIVITY RATIOS OF THE SKIN AND THE CORE MATERIAL ON THE RESPONSE OF THE VISCOELASTIC SANDWICH BEAM}

In the previous discussion, it is assumed that the ratio of moisture diffusivity of the skin to that of the core is 0.1 . In general, the moisture diffusivity of the skin/facings and core can vary in any ratio. The lower the moisture diffusivity of the skin, the longer it would take for the entire sandwich composite to attain the steady state. In addition, the moisture concentration at any point at a particular instant time can be significantly different with the different moisture diffusivity ratios of the skin and core. As a result of this the moisture dependent material properties can vary significantly with the different diffusivities of the skin and the core thus affecting the overall response of the sandwich composite. In this section, the effect of different moisture diffusivity ratios of the skin to that of core materials is being analyzed. Three different cases of the moisture diffusivity ratios have been considered.

Case (i) Moisture diffusivity ratio of the skin to that of the core is 1.0

Case (ii) Moisture diffusivity ratio of the skin to that of the core is 0.1

Case (iii) Moisture diffusivity ratio of the skin to that of the core is 0.01

Table 3.7 shows the values of the moisture diffusivities used for the skin and the core material. 
Table 3.7. Different moisture diffusivities of the skin and the core

\begin{tabular}{|c|c|c|}
\hline Moisture diffusivity & Moisture diffusivity & Moisture diffusivity \\
ratios & of skin, D $\left(\mathrm{mm}^{2} / \mathrm{sec}\right)$ & of core, D $\left(\mathrm{mm}^{2} / \mathrm{sec}\right)$ \\
\hline 1 & $1.06 \mathrm{E}-02$ & $1.06 \mathrm{E}-02$ \\
0.1 & $1.06 \mathrm{E}-03$ & $1.06 \mathrm{E}-02$ \\
0.01 & $1.06 \mathrm{E}-04$ & $1.06 \mathrm{E}-02$ \\
\hline
\end{tabular}

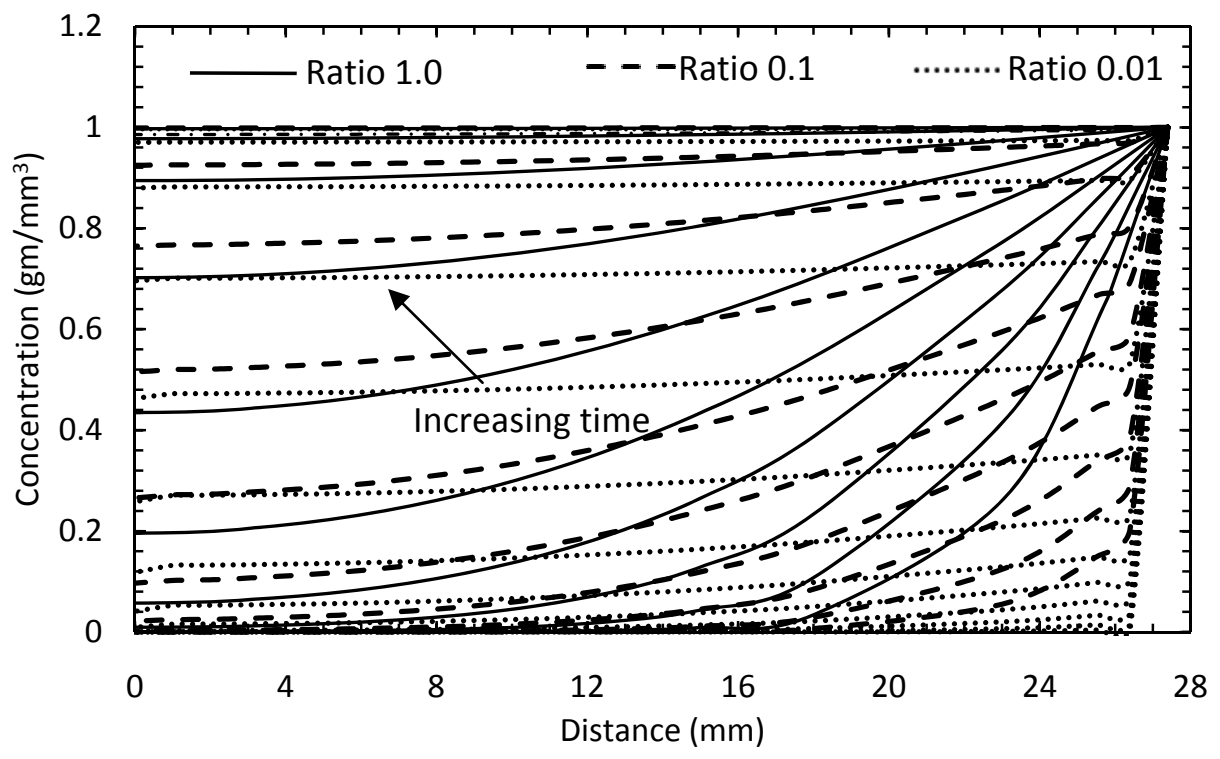

Fig. 3.26. Evolution of the moisture diffusion profile with different diffusivity ratios 
Fig 3.26 shows the evolution of the moisture concentration with different diffusivity ratios for an applied moisture concentration of $1 \mathrm{gm} / \mathrm{mm}^{3}$ at the surface of the top skin. As far as the transients are concerned, the concentration varies through the thickness of the composite differently. However, the asymptotic solution in all the three cases is the same and concentration reaches a value of 1 asymptotically when all the three ratios reach the steady state. It is observed that the moisture diffuses through the sandwich beam for the case (i) faster as compared to case (ii) and case (iii). Similarly, the moisture diffuses comparatively faster in case (ii) when compared to case (iii). The steady state for the case (i) is reached at 117 hours, for case (ii) is reached at 233 hours, and for case (iii) is reached at 932 hours. The different moisture diffusion profiles with the different moisture diffusivity ratios affect the overall behavior of the entire sandwich structure. At any time less than about 230 hours the magnitude of the field variables is always greater for the case (i) as compared to case (ii) and case (iii). For the case (i) the moisture diffuses at a faster rate as compared to the case (ii) and case (iii) and hence the material modulus is degraded faster. As a result of this higher displacements and strains are observed for the case (i). At any time greater than 230 hours the differences in the field variables of the case (i) as compared to case (ii) are almost negligible as the steady state is already reached for both the cases. This is because the viscoelastic materials posses a property of fading memory due to which the effect of the previous histories at a longer time becomes negligible. For any time greater than 932 hours, the differences in the field variables with the three diffusivity ratios are negligible as the steady state with all the three ratios is attained. 
Fig 3.27 shows the variations in transverse displacement $\left(\mathrm{U}_{2}\right)$ at different times for the three cases. At time $t=0.91$ hours the transverse displacement in the case (i) is $3 \%$ and $5 \%$ higher than the cases (ii) and case (iii) respectively. In the initial range of time this difference in the displacement values keep on increasing upto a particular time. At time $\mathrm{t}=3.6$ hours, the transverse displacement in the case (i) is about $6 \%$ and $11 \%$ higher in comparison to the case (ii) and case (iii) respectively. This signifies that the effect of different moisture diffusivity ratios is quite significant in the beginning times. At time $\mathrm{t}=29$ hours the transverse displacement in the case (i) is about $4 \%$ higher in comparison to the case (ii). Thus, there is a decrease in the percentage of increase in the transverse displacement as compared to the increment at time $\mathrm{t}=3.6$ hours. This signifies that the effect of different moisture diffusivity ratio of the case (i) as compared to case (ii) begins to diminish at time $\mathrm{t}=29$ hours. In contrast, the transverse displacement in the case (i) is about $16 \%$ higher in comparison to the case (iii). Thus, the effect of different diffusivity ratios for the cases (i) as compared to case (iii) is still on an increasing trend at time $\mathrm{t}=29$ hours. At time $\mathrm{t}=466$ hours the difference in the transverse displacements for the case (i) and case (ii) is less than $0.001 \%$ because the steady state for both the cases has been attained. Whereas the difference in the transverse displacement for the case (i) as compared to case (iii) is still about $1 \%$ as the steady state for the case (iii) is not yet attained. At time $\mathrm{t}=932$ hours the steady state is already attained for all the three moisture diffusivity ratios as a result of which negligible differences are observed. Thus, the effect of different diffusivity ratios is found to be prominent at the initial times but 

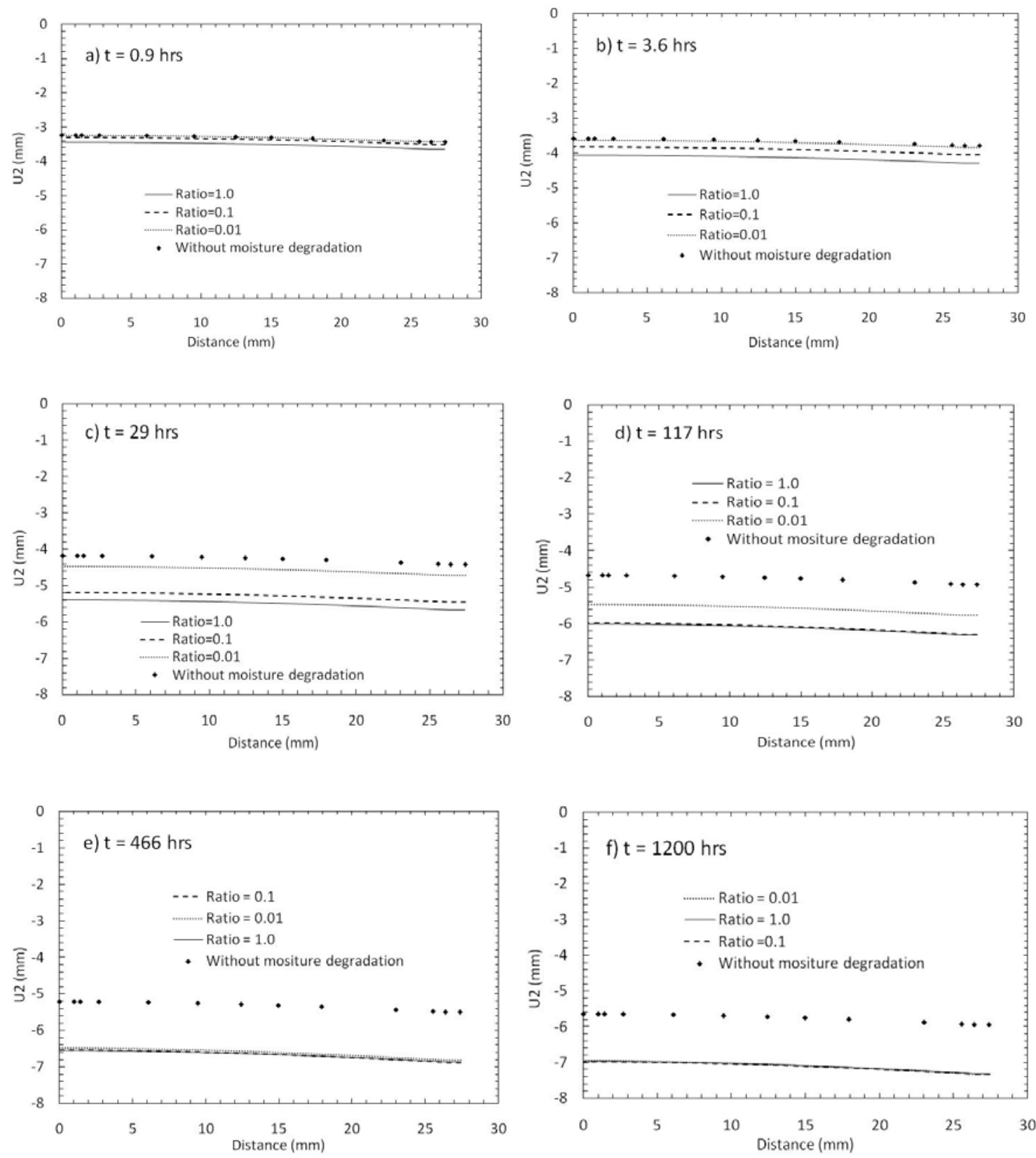

Fig. 3.27. Comparison of the transverse displacement field at the midspan of the sandwich beam for different moisture diffusivity ratios 
with the increase in time, this effect goes on decreasing and at any time greater than 932 hours the effect is almost negligible

Fig 3.28 shows the variations in the bending stresses $\left(\sigma_{11}\right)$ at different times for the three moisture diffusivity ratios. At time $t=0.91$ hours the magnitude of jump at the top interface for the case (i) is about $2.5 \%$ and $4 \%$ higher than the cases (ii) and case (iii) respectively. The magnitude of the jump at the bottom interface for the case (i) is about $2 \%$ and $3.5 \%$ higher as compared to the cases (ii) and case (iii) respectively. In the initial range of time, a continuous increase in the value of jump is observed upto a particular time. At time $\mathrm{t}=3.6$ hours, the magnitude of jump at the top interface for the case (i) is about $7 \%$ and $9 \%$ higher in comparison to the case (ii) and case (iii) respectively. At the bottom interface, the magnitude of jump for the case (i) is about $5 \%$ and $7.5 \%$ higher as compared to the case (ii) and case (iii) respectively indicating the impact of the different diffusivity ratios during the initial times. It is observed that the magnitude of jump at the top interface in all the three cases decreases with time for all times $\mathrm{t}$ greater than $29 \mathrm{hrs}$. At time $\mathrm{t}=117$ hours the magnitude of jump at the bottom interface in case (i) is $20 \%$ higher as compared to case (iii). Whereas the difference in the magnitude of jumps in case (i) and case (ii) is almost negligible for all times greater than 223 hours. This is attributing to the fact that the steady state is already reached in case (ii) at time 223 hours. At time $t=932$ hours the steady state is attained for all the three diffusivity ratios as a result of which negligible differences in the magnitude of jumps is observed. The effect of moisture on the other field variables is as shown in Figs 3.29 to 3.33 . 

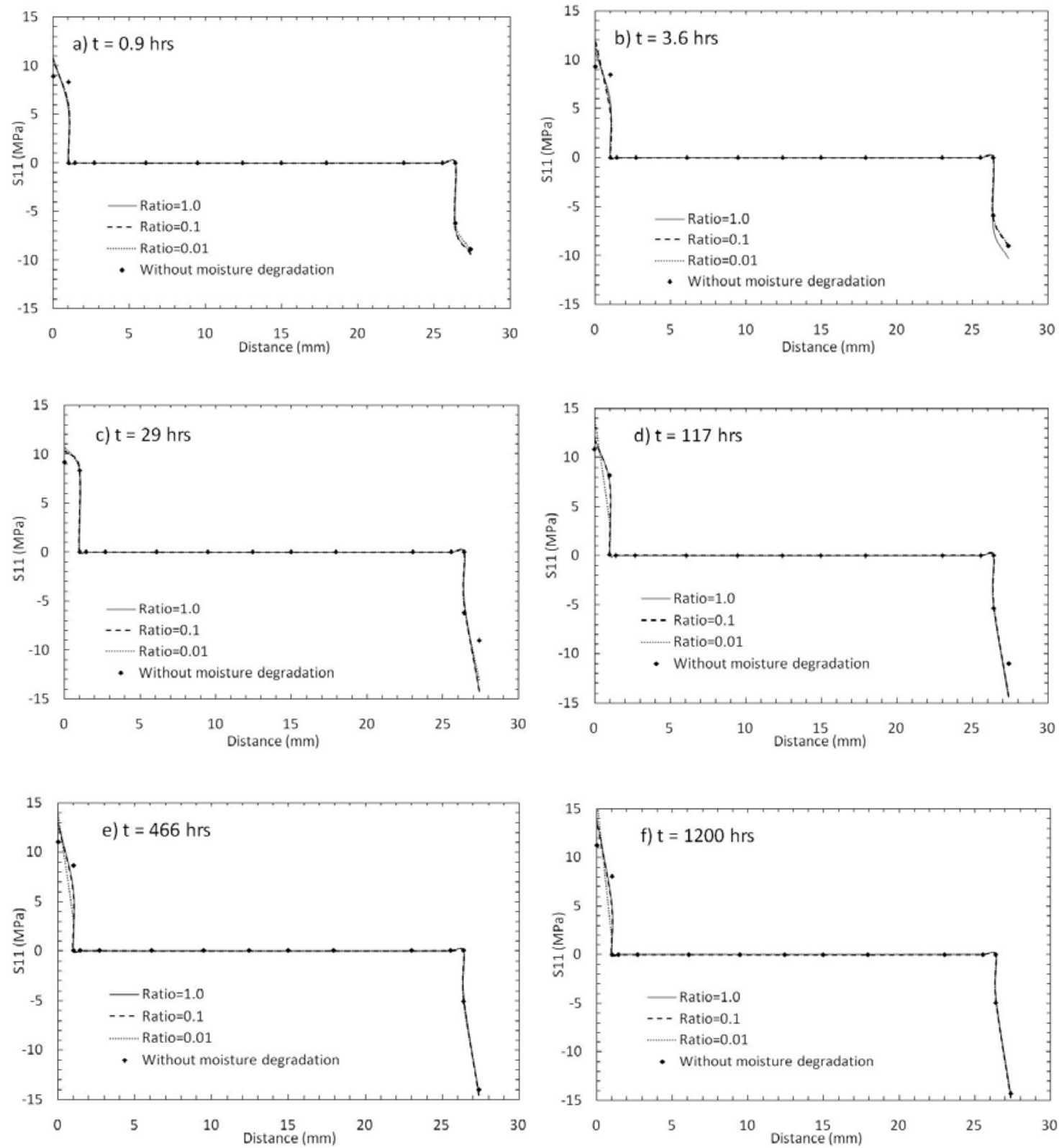

Fig. 3.28. Comparison of the axial stress field at a distance of $70 \mathrm{~mm}$ from the midspan of the sandwich beam for different moisture diffusivity ratios 

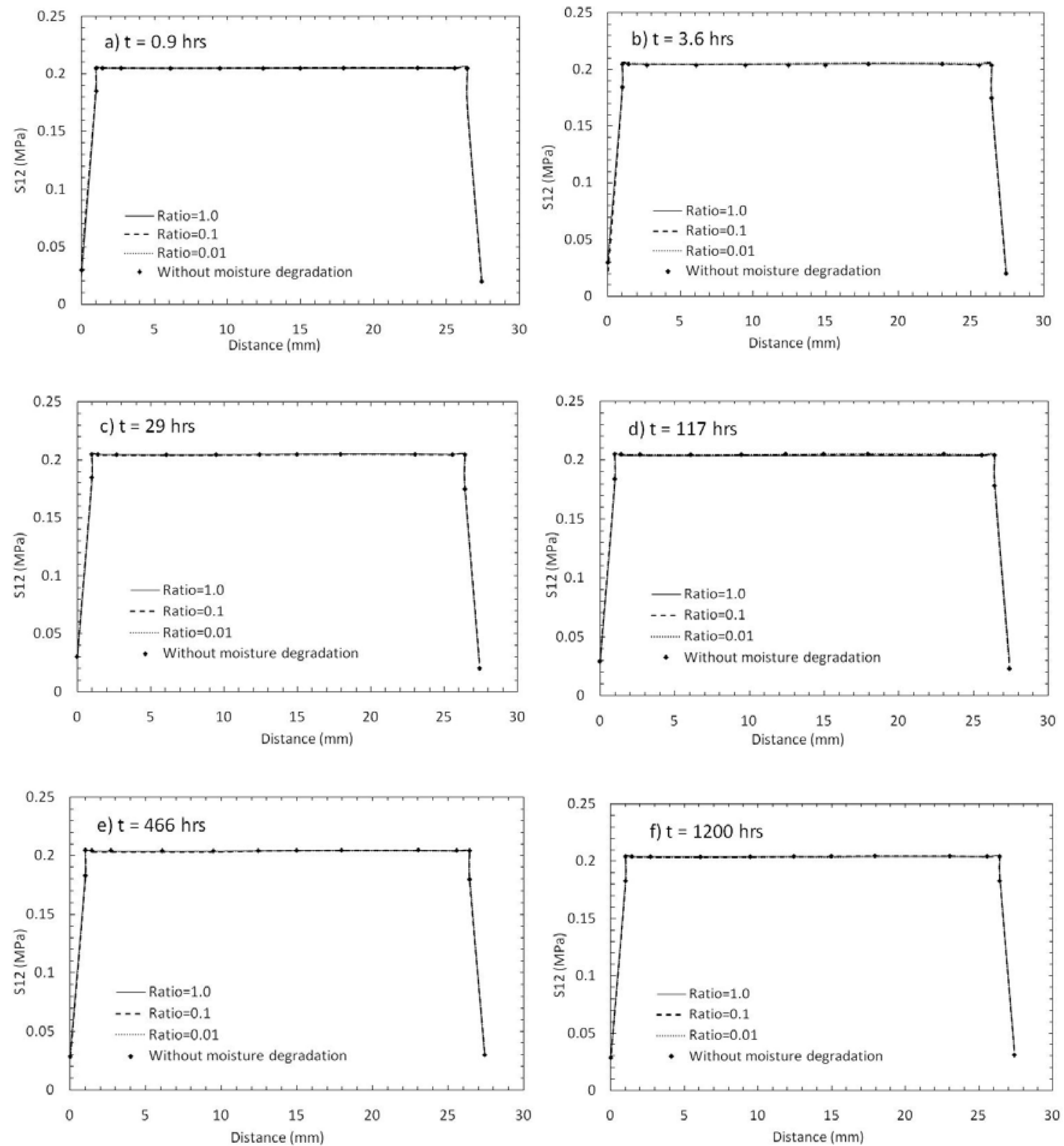

Fig. 3.29. Comparison of the shear stress field at a distance of $70 \mathrm{~mm}$ from the midspan of the sandwich beam for different moisture diffusivity ratios 

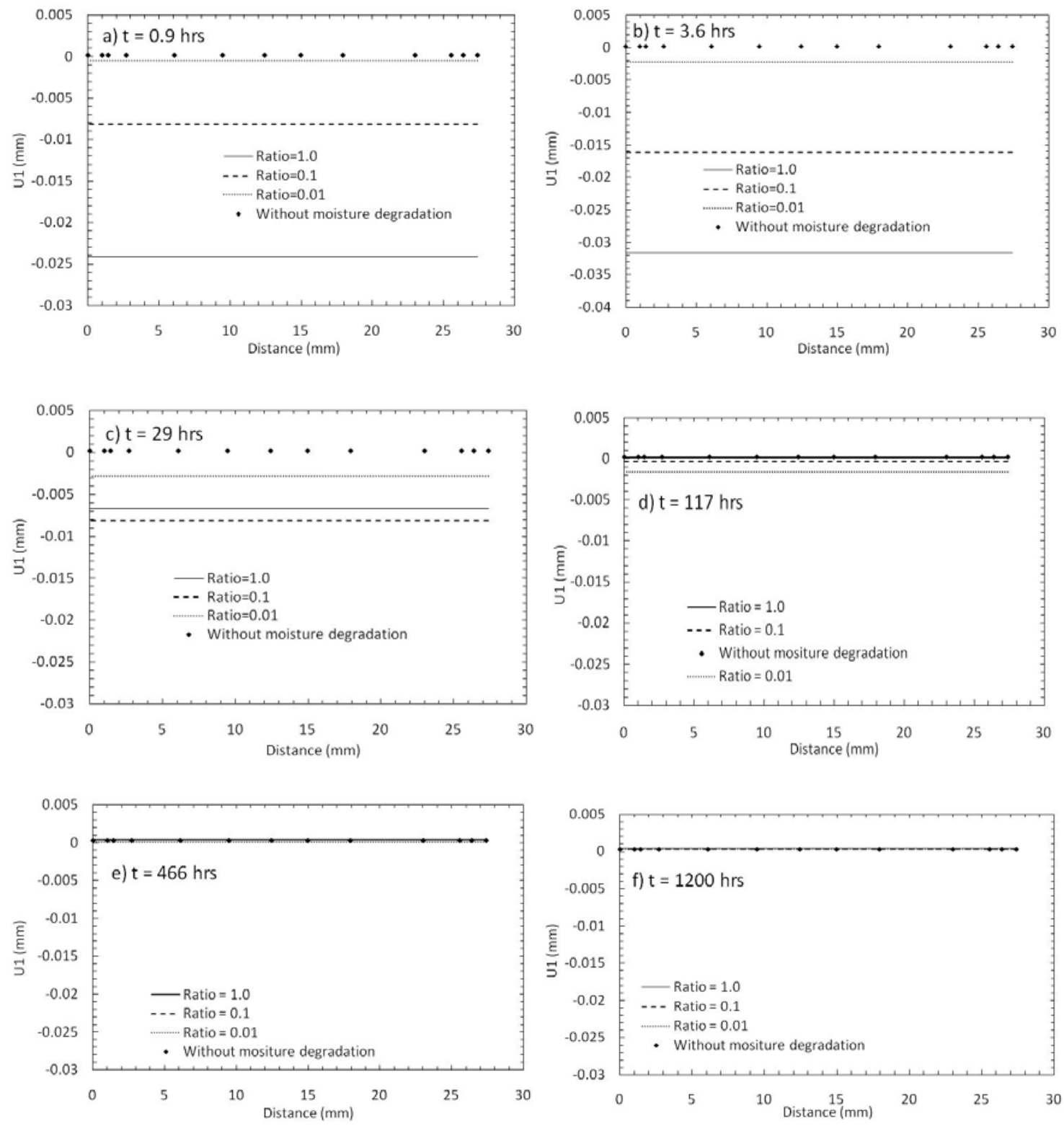

Fig. 3.30. Comparison of the longitudinal displacement field at the midspan of the sandwich beam for different moisture diffusivity ratios 

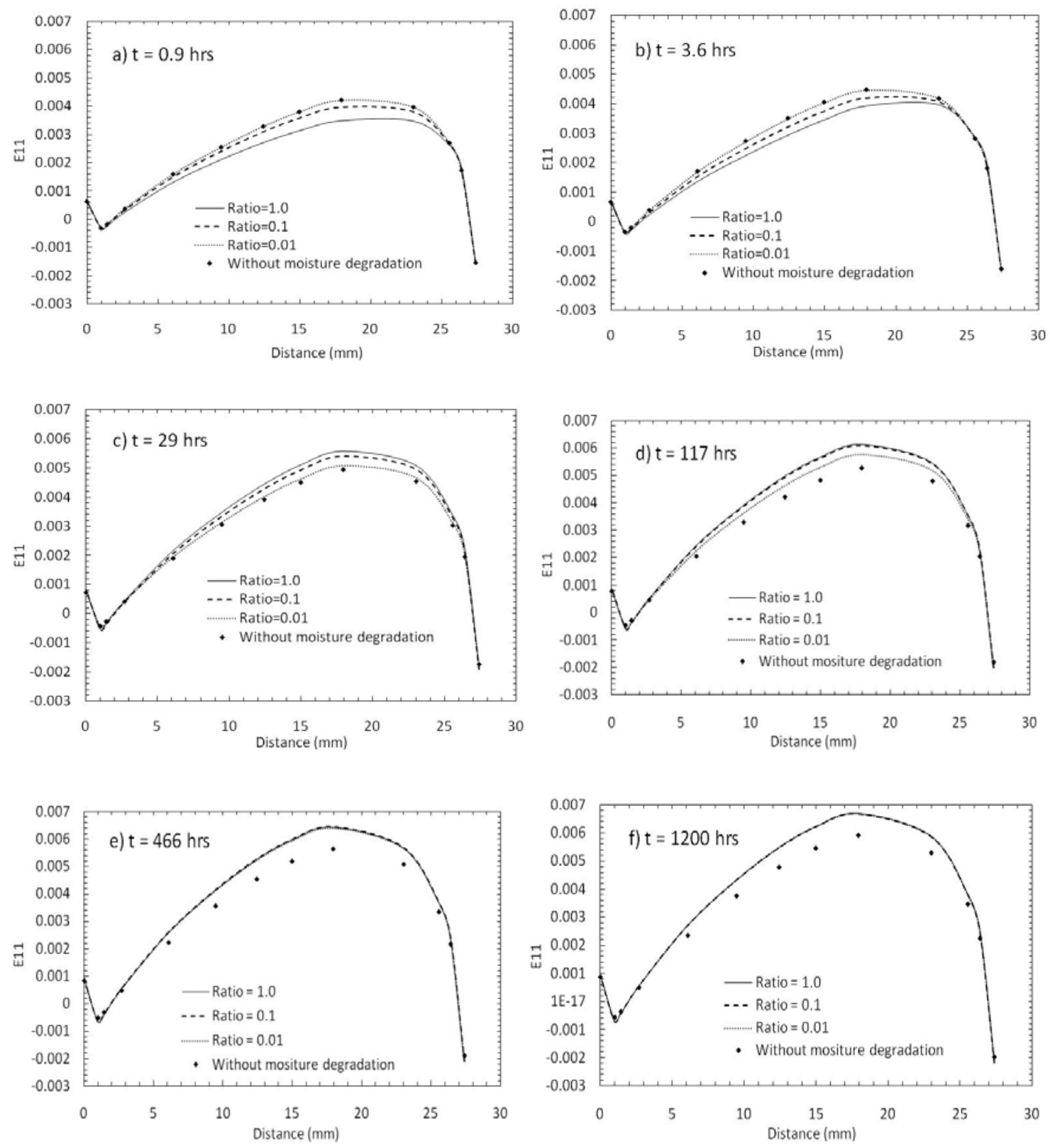

Fig. 3.31. Comparison of the longitudinal (axial) strain field at the midspan of the sandwich beam for different moisture diffusivity ratios 

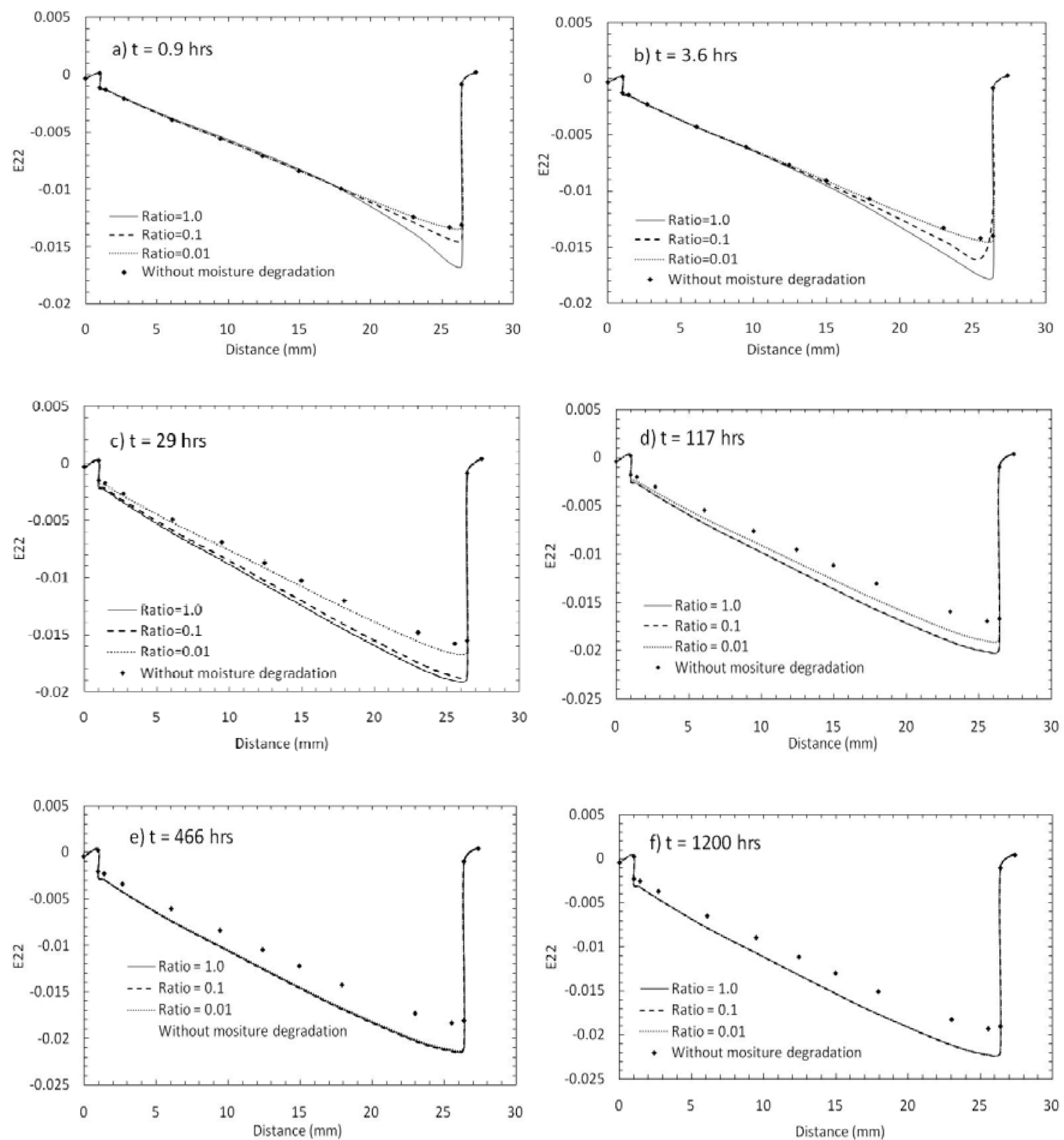

Fig. 3.32. Comparison of the transverse strain field at the midspan of the sandwich beam for different moisture diffusivity ratios 

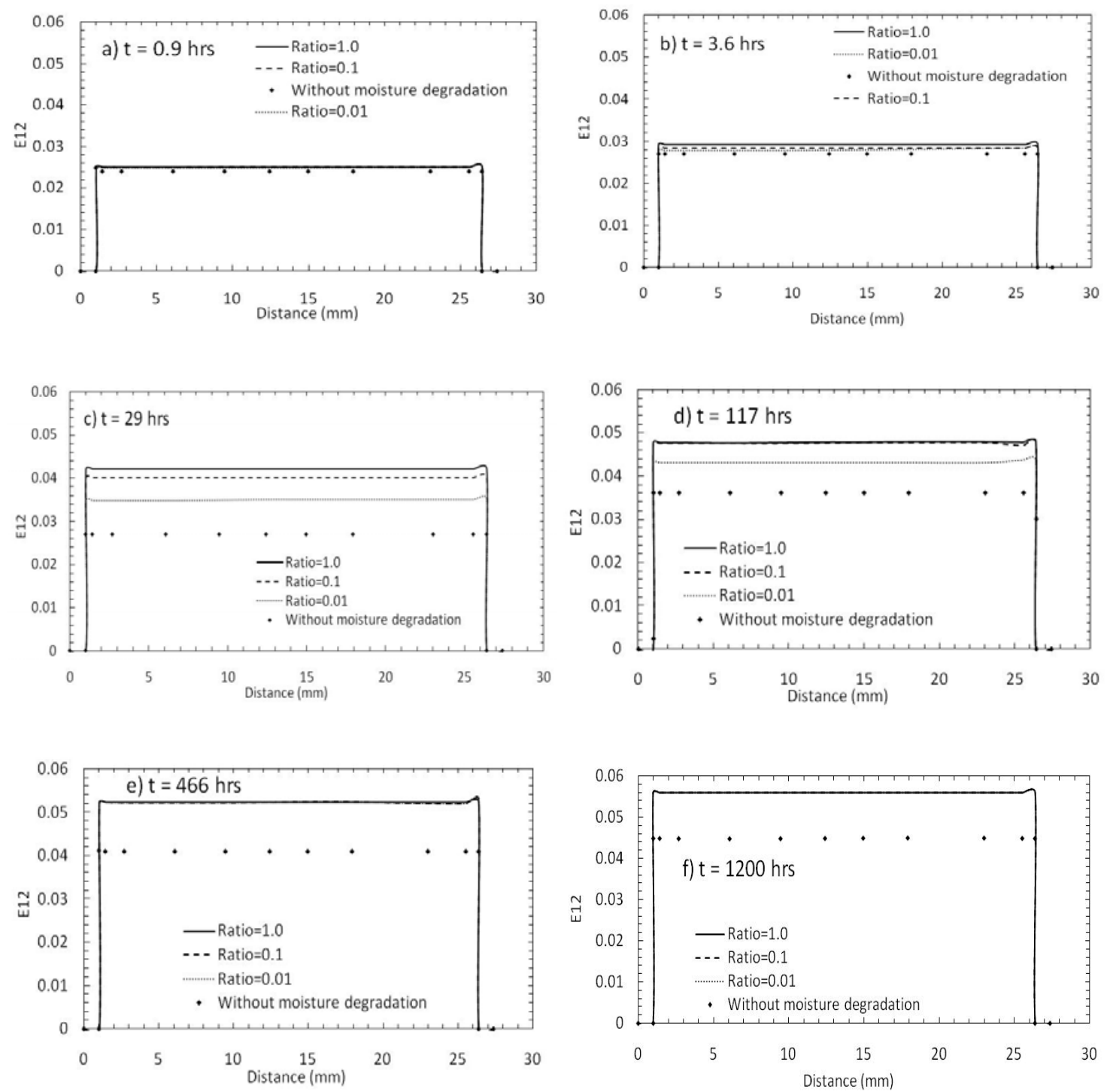

Fig. 3.33. Comparison of the shear strain field at a distance of $70 \mathrm{~mm}$ from the midspan of the sandwich beam for different moisture diffusivity ratios 


\subsubsection{INSTANTANEOUS AND TIME DEPENDENT MODULI BOTH MADE FUNCTIONS OF MOISTURE CONCENTRATION}

The entire discussion until now was based on the analysis performed with the instantaneous modulus of the viscoelastic foam core degrading with the moisture concentration. However, for the viscoelastic materials the time dependent modulus can also be a function of moisture concentration. This can have a significant impact on the overall behavior of the sandwich composite, depending upon the form in which the time dependent modulus degrades with the moisture concentration. The time dependent modulus in this study is assumed to degrade with moisture concentration. This is accounted through the non-dimensionalized parameter $g_{1}$ in the Eq. (2.3) as:

$$
g_{1}=1+\beta * \frac{c}{C_{0}}
$$

Here $C_{0}$ is the applied moisture concentration of $1 \mathrm{gm} / \mathrm{mm}^{3}$ and $\beta$ is a constant equal to 0.62 .

At the different depths through the thickness of the sandwich beam, the creep compliance of the viscoelastic polyurethane foam core is allowed to change with the moisture concentration at various times. In this study, the overall response of the sandwich structure under the three point bending test, with both the instantaneous and transient modulus of the viscoelastic polyurethane foam core degrading with moisture is analyzed. The Eq. (3.11) governs the degradation of the instantaneous part, while the transient part degrades as per the Eq. (3.14). Fig 3.34 shows the graph of the moisture dependent creep compliance at half the thickness $(\mathrm{x}=13.7 \mathrm{~mm})$ of the sandwich beam for 
the two cases shown below. A specific case of the moisture diffusivity ratio of 0.1 (skin to core) is considered for the two cases.

Case (i) Only the instantaneous modulus of the viscoelastic polyurethane foam core degrades with the moisture concentration as per the Eq. (3.11).

Case (ii) Both the instantaneous and transient moduli of the viscoelastic polyurethance foam core degrade with the moisture concentration as per the Eq. (3.11). and Eq. (3.14). respectively.

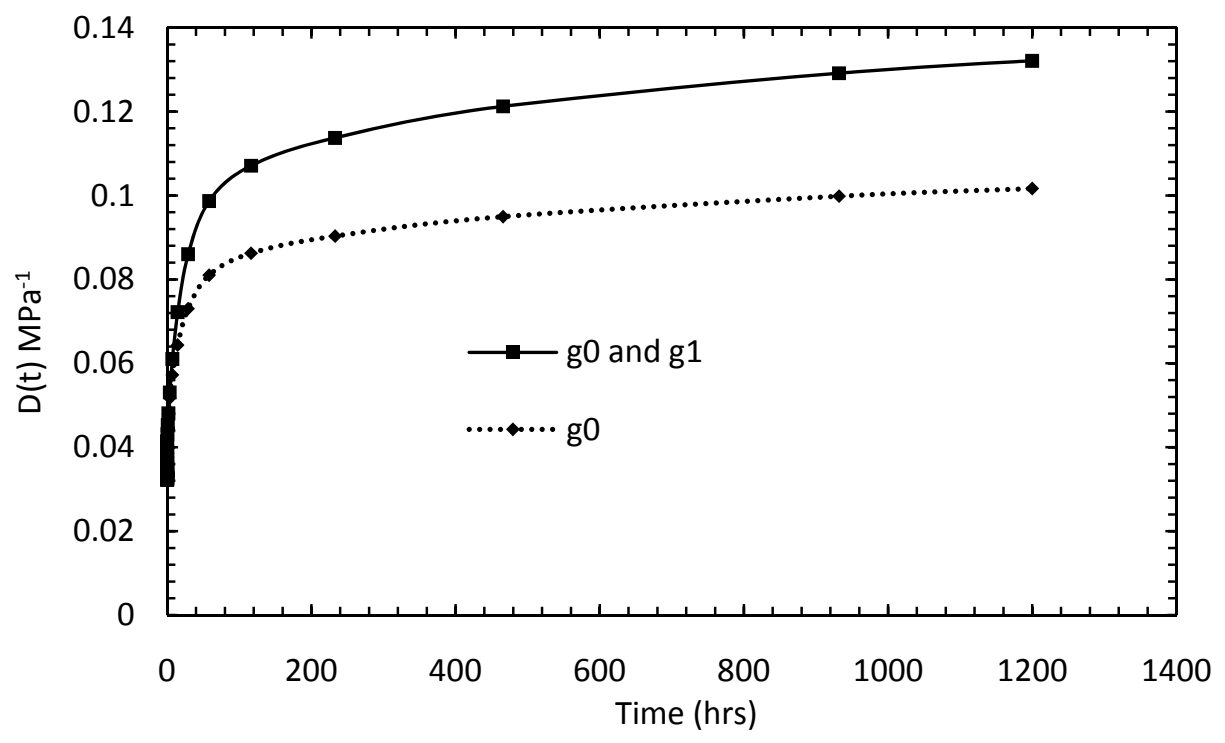

Fig. 3.34. Creep compliance for the two cases at $\mathrm{x}=13.7 \mathrm{~mm}$ 
At the initial times the degradation of the transient modulus with the moisture concentration is not very significant. As a result, not much difference is observed in the field variables for the case (ii) when compared to case (i). But with the increase in the time the moisture diffusion through the sandwich beam progresses and the creep compliance for the case (i) is significantly different than the case (ii). As a result, differences in the field variables for the case (ii) as compared to case (i) are observed. Once the steady state for the moisture diffusion process is reached, for all the times ahead nearly a constant difference in the field variables is observed when the two cases are compared as expected.

As shown in Fig 3.35 at time $t=3.64$ hours an increase of around $2.5 \%$ in the transverse displacement for the case (ii) as compared to case (i) is observed. With the increase in time this difference increases. At time $\mathrm{t}=116$ hours the increase is about $9.5 \%$ in the case (ii) as compared to case (i). At any time greater than 233 hours (the time required to reach the steady state for the moisture diffusion process), a nearly constant increase of around $8.5 \%$ is observed in case (ii) when compared to case (i). The instantaneous and the transient modulus for any time greater $\mathrm{t}=233$ hours do not change with moisture concentration as the steady state is already attained. This results into a constant difference in the values of the transverse displacement for any time greater than 233 hours when the case (ii) is compared with case (i). However, the deformation in the two cases are still progressing with time. 

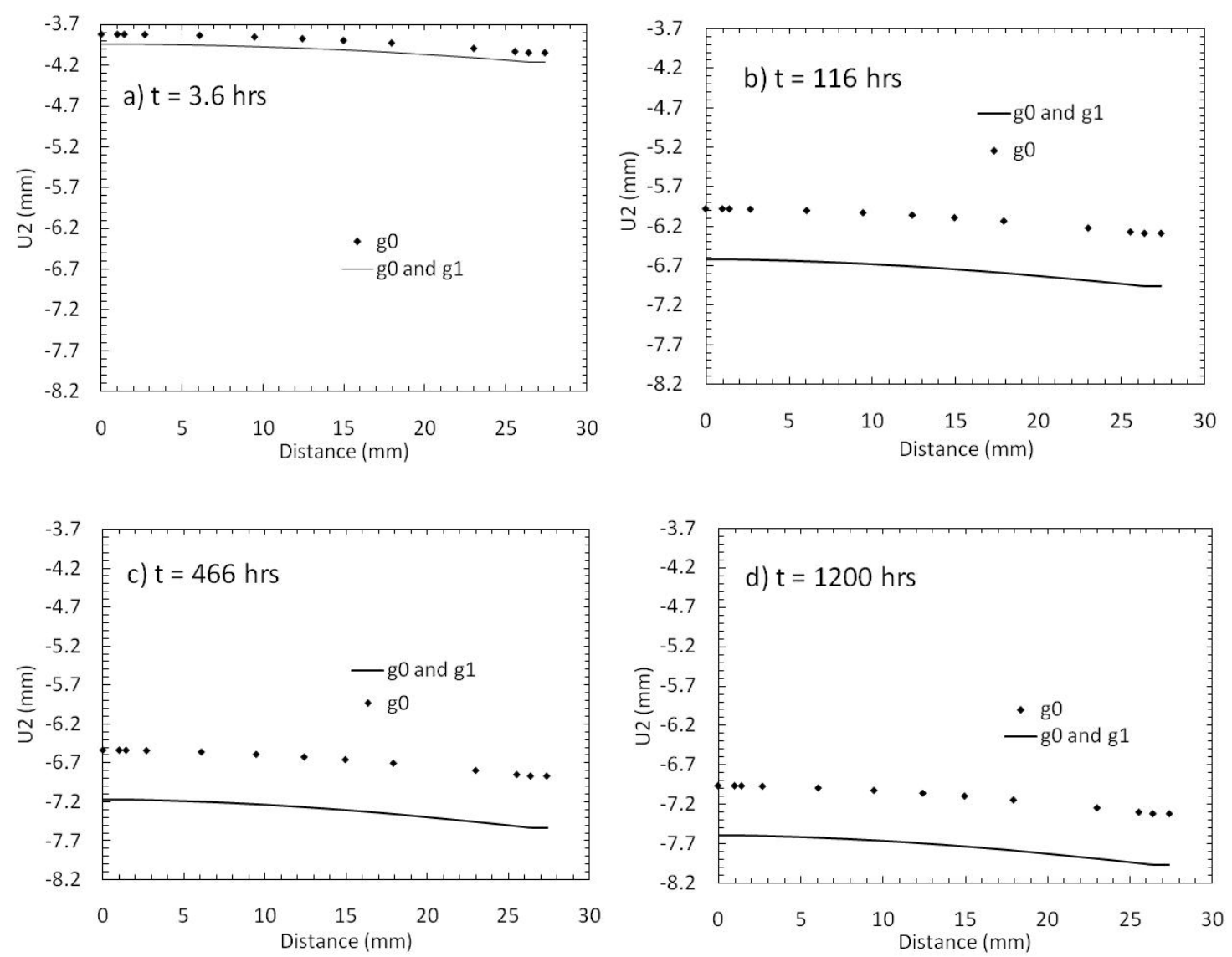

Fig. 3.35. Comparison of the transverse displacement field at the midspan of the sandwich beam for case with moisture dependent instantaneous part and the case with moisture dependent instantaneous and time dependent part

Fig 3.36 shows the variation in the bending stress with time. As explained before a jump in the bending stress $\left(\sigma_{11}\right)$ is observed at both the top and the bottom interfaces in both the cases (i) and (ii). At time $t=3.64$ hours the magnitude of jump at the top interface ( $x=26.4 \mathrm{~mm}$ ) for the case (ii) is about $2 \%$ higher in comparison to the case (i). At the bottom interface $(\mathrm{x}=1 \mathrm{~mm})$ magnitude of the jump is about $2.5 \%$ higher for the case (ii) as compared to case (i). It is worth observing that the magnitude of jump at the top interface decreases with the increase in time for both the cases. Whereas the 
magnitude of jump in the bottom interface for both cases increases upto time $\mathrm{t}=116$ hours and then decreases for the later times. It is also observed that the magnitude of the maximum stresses (in the facings) increase with the increase in time in both the cases.
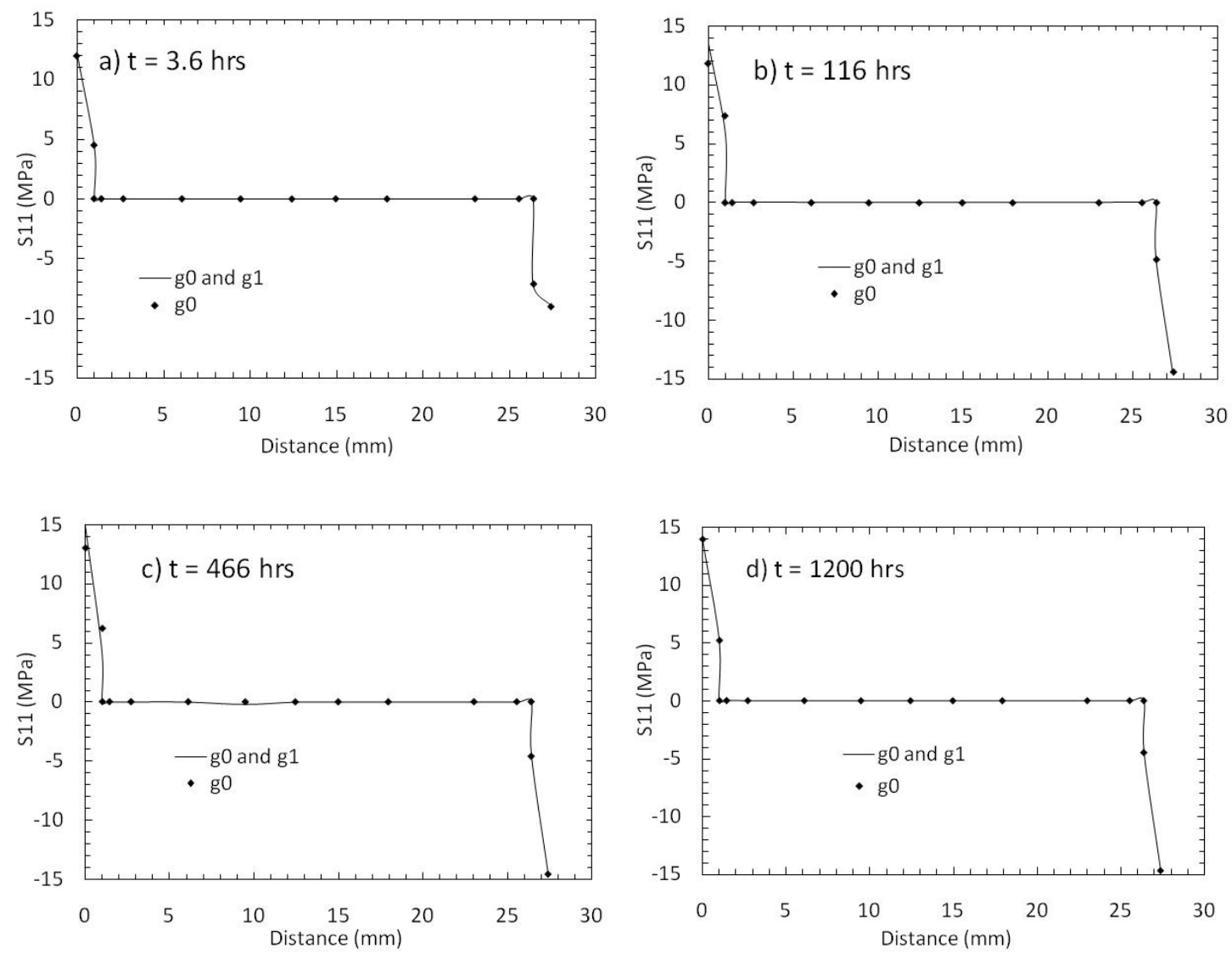

Fig. 3.36. Comparison of the bending stress field at a distance of $70 \mathrm{~mm}$ from the midspan of the sandwich beam for case with moisture dependent instantaneous part and the case with moisture dependent instantaneous and time dependent part 

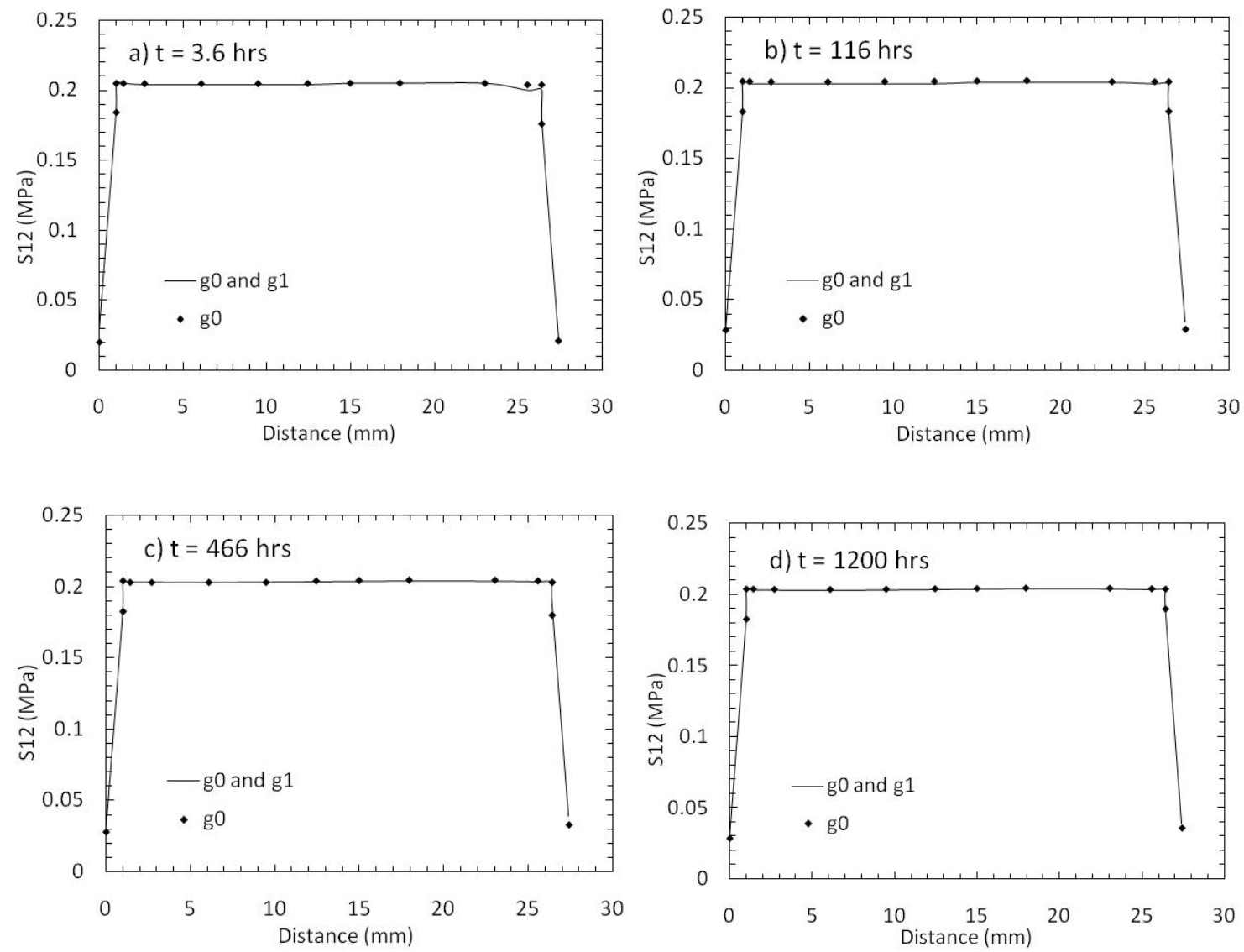

Fig. 3.37. Comparison of the shear stress field at a distance of $70 \mathrm{~mm}$ from the midspan of the sandwich beam for case with moisture dependent instantaneous part and the case with moisture dependent instantaneous and time dependent part

Figs 3.37 to 3.41 show the variations in the other field variables with time. 

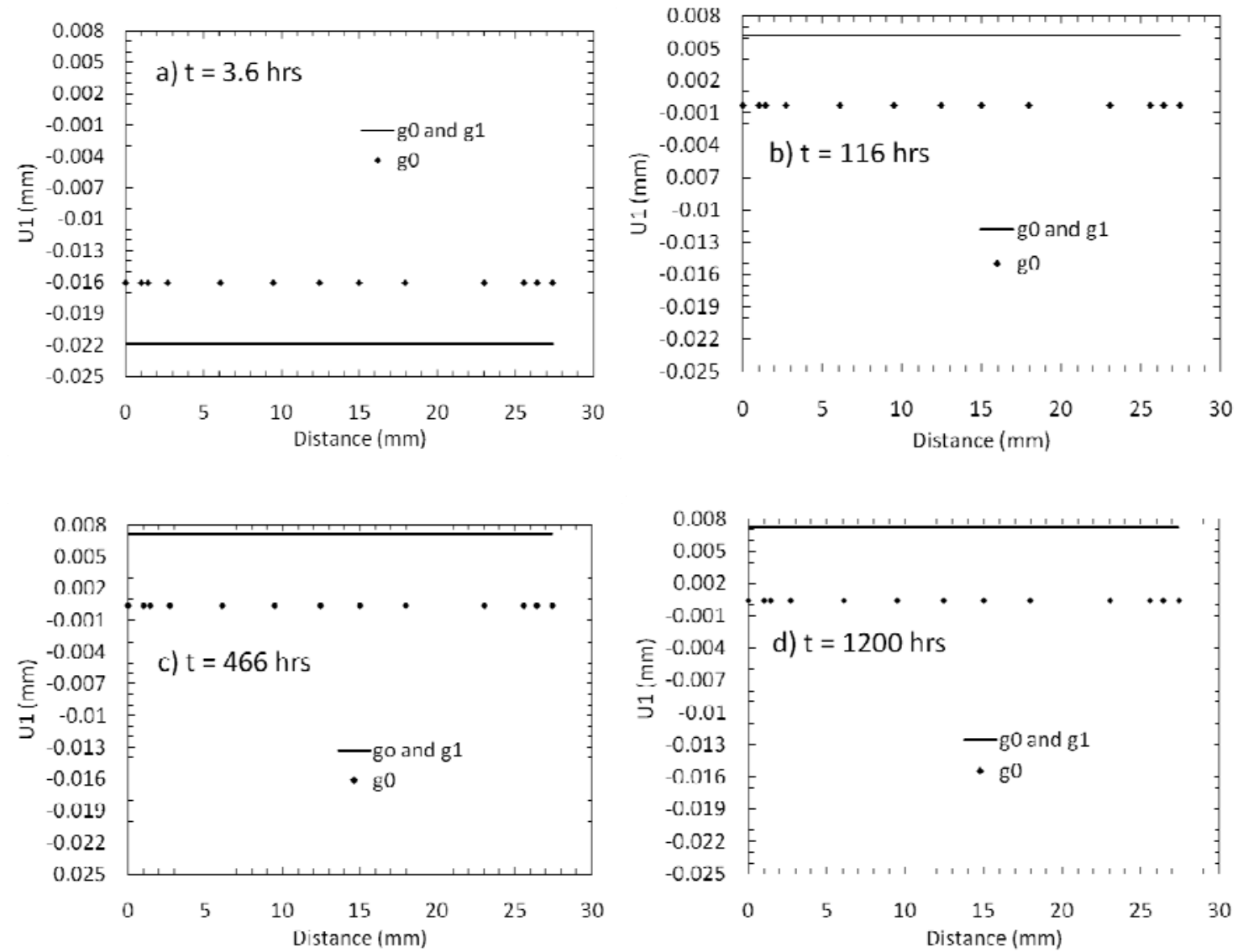

Fig. 3.38. Comparison of the longitudinal displacement field at the midspan of the sandwich beam for case with moisture dependent instantaneous part and the case with moisture dependent instantaneous and time dependent part 

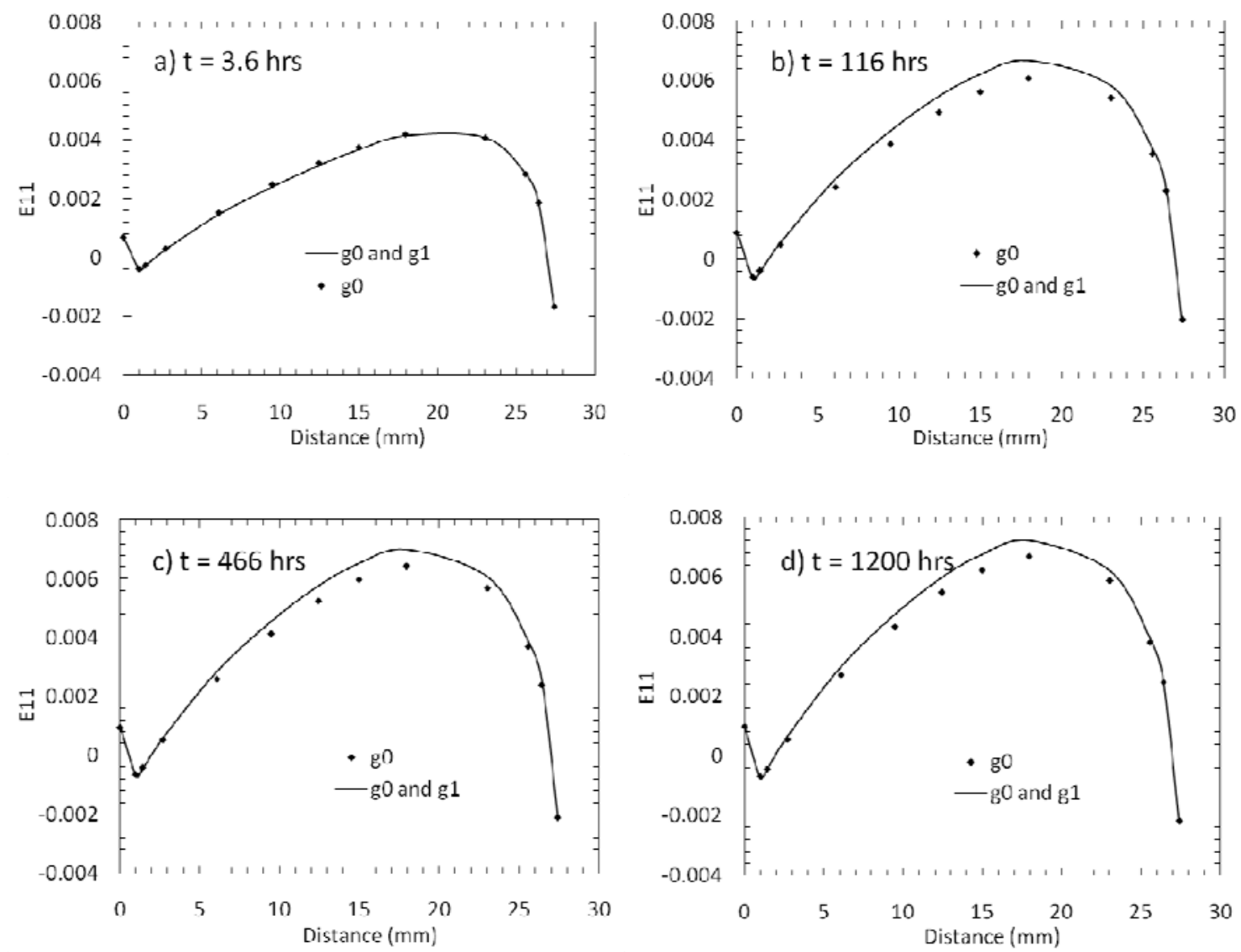

Fig. 3.39. Comparison of the longitudinal strain field at the midspan of the sandwich beam for case with moisture dependent instantaneous part and the case with moisture dependent instantaneous and time dependent part 

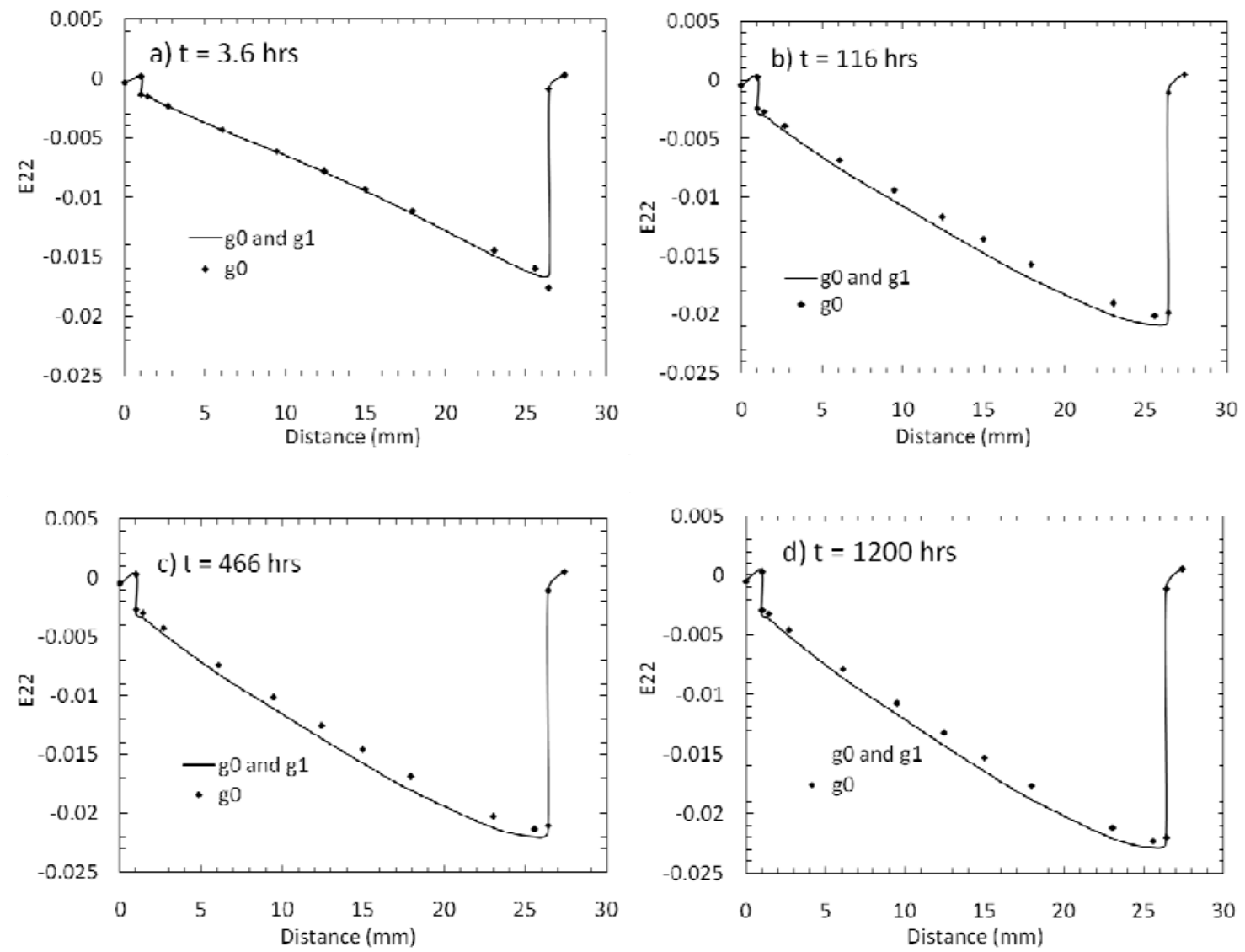

Fig. 3.40. Comparison of the transverse strain field at the midspan of the sandwich beam for case with moisture dependent instantaneous part and the case with moisture dependent instantaneous and time dependent part 

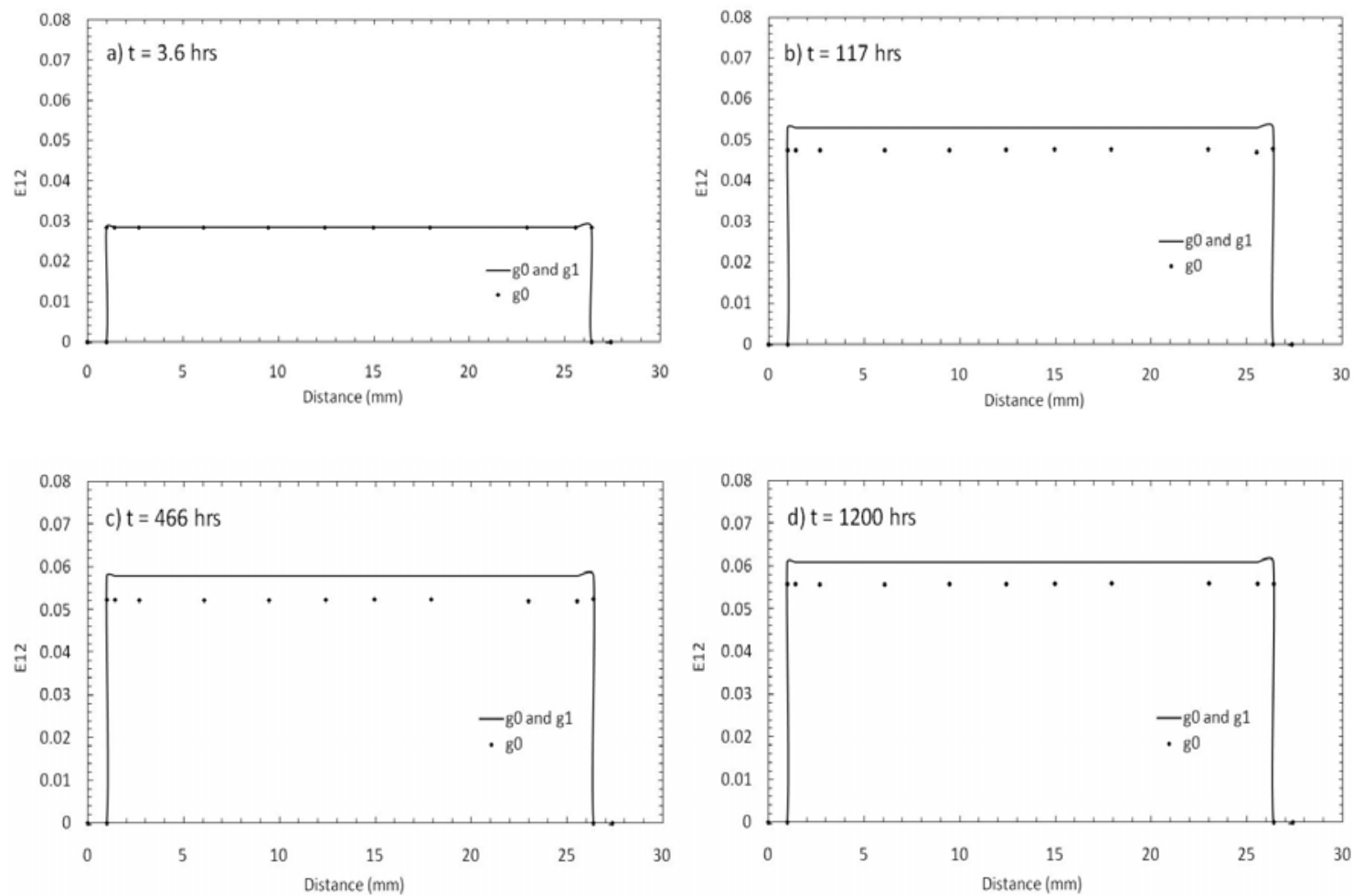

Fig. 3.41. Comparison of the shear strain field at a distance of $70 \mathrm{~mm}$ from the midspan of the sandwich beam for case with moisture dependent instantaneous part and the case with moisture dependent instantaneous and time dependent part 


\section{CHAPTER IV}

\section{FINITE ELEMENT ANALYSIS OF THE SKIN-CORE DELAMINATION AND THE HYGROSCOPIC SWELLING IN SANDWICH COMPOSITES}

This chapter presents the structural analysis of the sandwich composites under the combined moisture diffusion and mechanical loading. It has already been shown in the previous chapters that moisture is found to have pronounced effects on the deformation of the viscoelastic sandwich composites. In the first section, the effect of moisture diffusion delamination between the skin-core interface in a sandwich beam under double cantilever beam (DCB) and the tilted sandwich debond (TSD) tests is studied. The sandwich beam is composed of orthotropic S2-glass/epoxy laminate and linear viscoelastic polyurethane foam core. The FM73M adhesive polymer is used to glue the skin and the core at the interface of the sandwich structure. The material moduli of the core and the glue at a particular time are functions of moisture concentration at that instant time. The second part of this chapter deals with the hygroscopic swelling in sandwich panels. The sandwich panels are fixed at the four sides. Significant amount of stresses and strains are induced in the sandwich panels due to the moisture concentration. The viscoelasticity in the sandwich systems causes gradual change in the deformations. 


\subsection{DELAMINATION AT THE SKIN-CORE INTERFACE OF THE SANDWICH COMPOSITE}

Adhesive polymers are widely used for joining structural components made of similar and dissimilar materials. The adhesive bonds provide flexibility in design, weight reduction, cost reduction, and more uniform stress distribution over an entire bonded surface. Adhesive polymers are also widely used in the aircraft structural components, automotive components, naval transportations and railway carriages for joining composite to steel. One of the important requirements of these polymeric adhesives is its durability and capability to sustain mechanical loads under various environmental conditions of temperature and moisture. Adhesive polymer is also used in gluing the skin-core interface of a sandwich composite. Because the sandwich composites may be exposed to severe temperature and moisture conditions depending upon the application, it becomes essential to study the delamination behavior at the skincore interface under such hostile environmental conditions.

Berggreen et al. [32] analyzed the debonding between core and face sheets in foam-cored sandwich structures. A FEM based numerical model for the predictions of the propagation of debond damage is developed and validated. Face tearing experiments are carried out for structures with three different core densities. The developed FEM model is validated using the experimental data. It was observed that for the low core density sandwich composites, the crack propagated at the interface just above the core, whereas for the higher density cores, the crack tends to propagate in the face sheets (laminate) itself. Saha et al. [33] analyzed the effect of addition of nanoparticles to the 
foam core on the debond fracture behavior of the sandwich panels. It was concluded that the infusion of nanoparticles in the foam core improved the adhesion property and enhanced the debond fracture toughness of the sandwich composites. Veazie et al. [34] investigated the interfacial fracture toughness of E-Glass/Vinylester facesheets, closedcell PVC core sandwich composites submersed in the sea-water for 5000 hours. A 50\% reduction in the interfacial fracture toughness as compared to the dry specimen was observed under the double cantilever beam test. Scudamore and Cantwell [35] performed experiments to analyze the skin-core interfacial fracture properties of a number of dry and moisture-conditioned sandwich structures under the three point bending test. Four sandwich specimens with different skin and core materials were tested. It was observed that the prolonged exposure to the seawater was found to affect the bond between the skin and the core, facilitating crack advance along the skin-core interface. It was concluded that great care should be exercised before selecting sandwich structures for hostile marine environment.

In all the previous chapters a perfect bonding at the skin-core interface was assumed. With the use of the polymeric adhesive at the skin-core interface there is a possibility that delamination at the interface may occur under severe loading conditions. In majority of the work done till date, the delamination at the skin-core interface is analyzed under static loading conditions for a short span of time. This study is performed to analyze the transient effect of moisture diffusion on the delamination at the interface under the creep loading for about 1200 hours (greater than the steady state time of 1150 hours for the moisture diffusion process). A sandwich beam composed of 
orthotropic S2-glass epoxy laminate and linear viscoelastic polyurethane foam core under the combined moisture and mechanical stimuli is studied. The material properties of the facing and the core are as shown in the Tables 4.1 and 3.2 (Chapter III) respectively. The instantaneous part $\left(\mathrm{E}_{0}\right)$ of the polyurethane foam core is assumed to degrade linearly with the moisture concentration as per the Eq. (3.11). The facings and the core material are glued together by the FM73M adhesive. The calibrated timedependent parameters (Prony series) representing the creep compliance and the elastic properties for the FM73M [36] are given in the Table 4.2. The constitutive relation stated in Eq (2.3) is used to model the material response of the glue. It is also assumed that the instantaneous part $\left(\mathrm{E}_{0}\right)$ of the FM73M adhesive degrade linearly with moisture concentration as per the Eq. (3.11). In this case the value of $\alpha$ is chosen to be $1335 \mathrm{MPa}$. The moisture dependent elastic moduli for the FM73M is then written as:

$$
E_{0}=\frac{1}{D_{0}}=E_{0}-1335 * \frac{c}{C_{0}}
$$

Here $\mathrm{E}_{0}=2710 \mathrm{MPa}$ is the instantaneous modulus of the FM73M adhesive and $c$ $\left(\mathrm{gm} / \mathrm{mm}^{3}\right)$ is the moisture concentration. The parameter $\mathrm{g}_{0}$ in Eq. (3.12) that measures the moisture dependent elastic compliance can therefore be written as:

$$
g_{0}=\frac{E_{0}}{\left(E_{0}-1335 * \frac{c}{C_{0}}\right)}
$$


Table 4.1. Elastic properties of S2-glass epoxy laminate

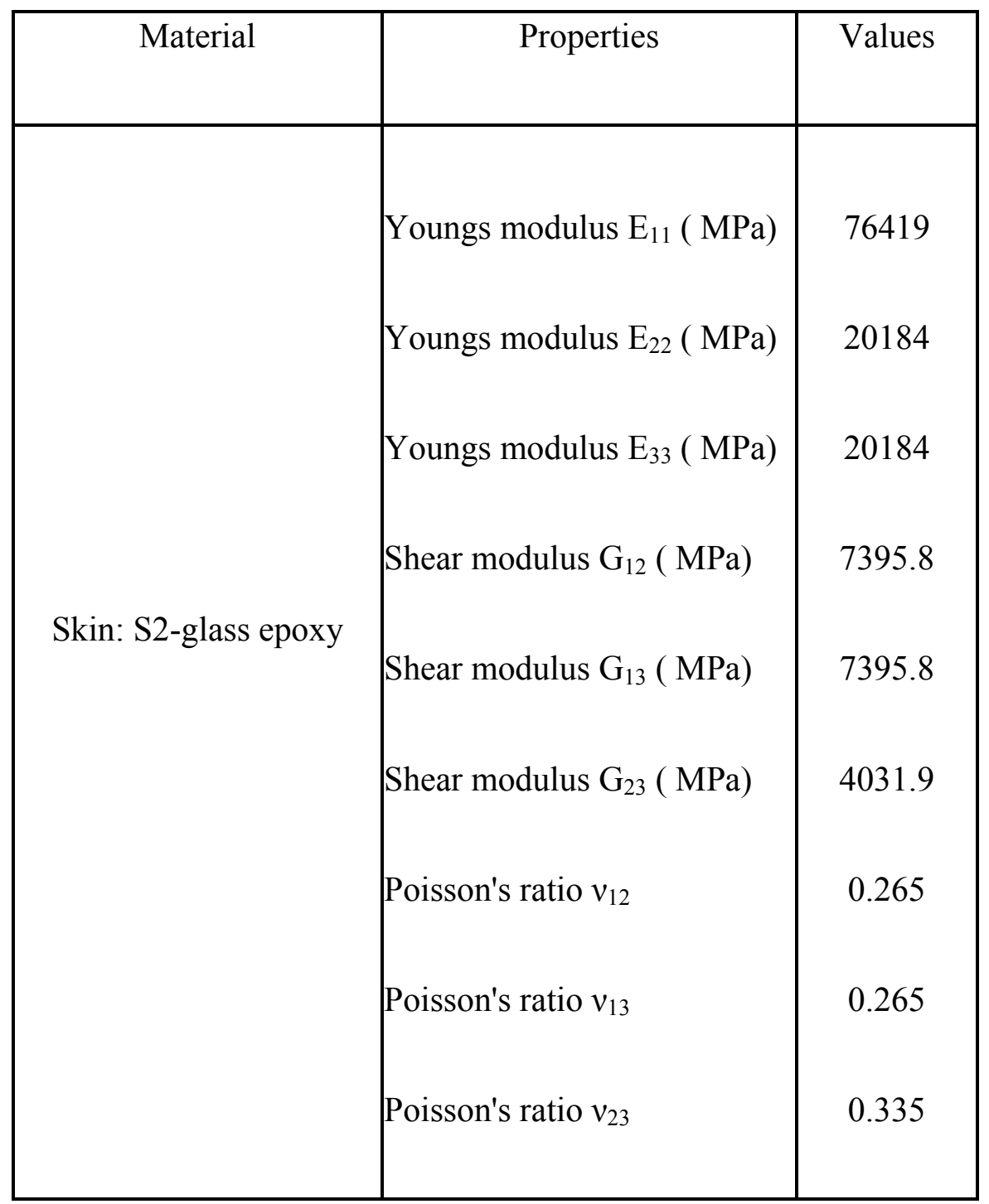


Table 4.2. Elastic properties and Prony series coefficients for FM73M

\begin{tabular}{|c|c|c|}
\hline $\mathrm{N}$ & $\lambda_{n}\left(\mathrm{sec}^{-1}\right)$ & $D_{n} \times 10^{-6}\left(\mathrm{MPa}^{-1}\right)$ \\
\hline 1 & 1 & 3.70 \\
3 & $10^{-1}$ & 40.7 \\
4 & $10^{-2}$ & 33.3 \\
5 & $10^{-3}$ & 55.6 \\
6 & $10^{-4}$ & 259 \\
7 & $10^{-5}$ & 1000 \\
8 & $10^{-6}$ & 3810 \\
9 & $10^{-7}$ & \\
\hline & $\mathrm{E}_{0}=2710 \mathrm{Mpa}$ & \\
\hline
\end{tabular}

The analysis is performed using the FE framework. The purpose of the numerical study is to simulate the crack initiation time and the cumulative crack length under the effect of moisture degradation. The analysis is performed using two different 
testing procedures named DCB and TSD developed by Carlsson [37, 38]. Fig 4.1 (a) and (b) shows the configuration for the DCB and TSD procedures respectively.

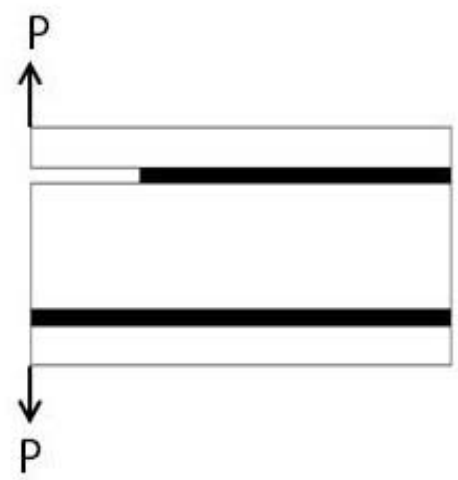

a)

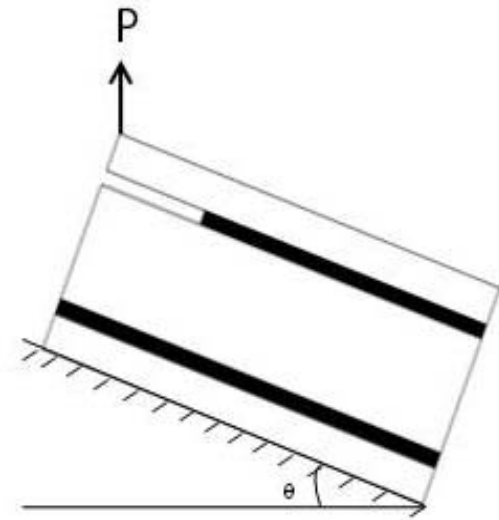

b)

Fig. 4.1. a) DCB test b) TSD test

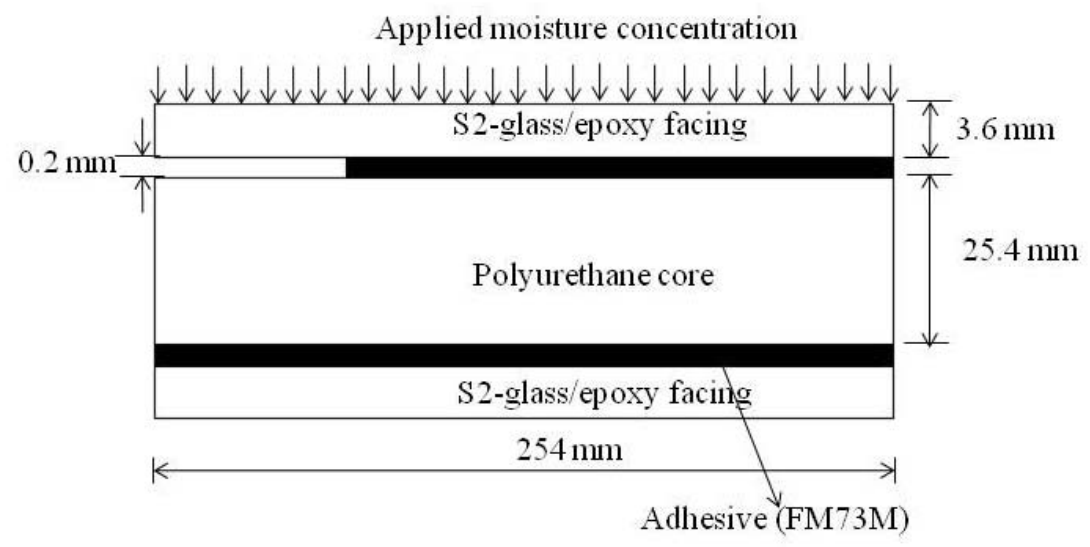

Fig. 4.2. A schematic of the sandwich composite glued together with adhesive 


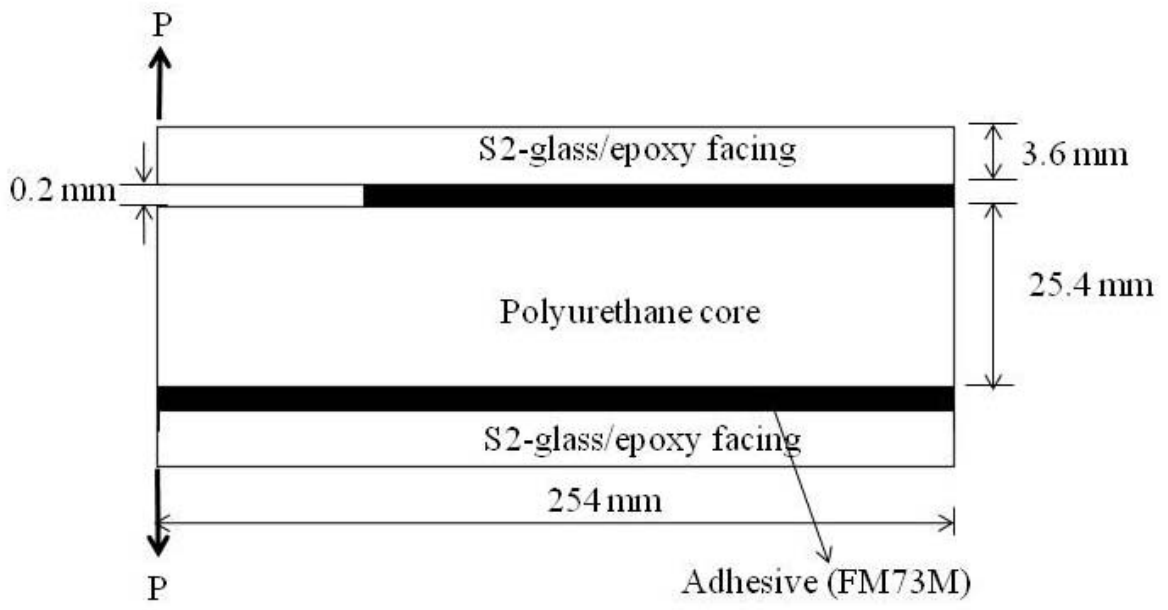

Fig. 4.3. A schematic of the double cantilever beam

The analysis is performed using the DCB test on the sandwich beam (254 mm X $32.6 \mathrm{~mm}$ X $38.1 \mathrm{~mm}$ ) [39] composed of S2-glass epoxy laminated facings and linear viscoelastic polyurethane foam core with an initial crack length of $50 \mathrm{~mm}$. The coupled analysis is performed in two sequential steps. In the first step, the moisture diffusion profile through the sandwich beam is obtained by applying the moisture concentration at the top surface of the skin as shown in the Fig 4.2. To simulate the moisture diffusion process using FE code, (DC2D4) 2D diffusivity elements are used. With the moisture diffusivity of the skin and the core assumed to be to $1.06 \mathrm{E}-3 \mathrm{~mm}^{2} / \mathrm{sec}$ and $1.06 \mathrm{E}-2$ $\mathrm{mm}^{2} / \mathrm{sec}$ respectively the steady state of the moisture diffusion process for the applied moisture concentration of $1 \mathrm{gm} / \mathrm{mm}^{3}$ is reached at around 1150 hours. Once the moisture distribution profile throughout the beam is obtained, mode I failure analysis is performed on the double cantilever beam (DCB) specimen as shown in the Fig 4.3. For this 
purpose, a 2D plane strain condition is imposed. To simulate the structural problem of DCB using FE code, (CPE4) plain strain elements are used. Total 5 and 66 numbers of meshes are generated along the adhesive's thickness and bonded length, respectively. Cohesive elements are applied along the predetermined failure path, which is located at (a) the interface between the top skin and the core (i.e. between the skin and adhesive and between core and adhesive) and (b) within the adhesive itself. The last four nodes near the fix end are tied, no longer allowing the crack propagating. Thus, complete debonding is determined once the current crack tip reaches the tied node. The crack opening displacement (COD) failure criterion, available in the ABAQUS FE code [40], is first used to evaluate crack initiation and propagation:

$$
f=\frac{\delta}{\delta_{c}}
$$

Here $\delta$ is the current (measured) normal displacement across the cohesive surface and $\delta_{c}$ is the critical opening displacement. The failure a criterion in the Eq. (4.3) is monitored at the distance of $3 \mathrm{~mm}$ ahead the current crack tip. When the failure criterion reaches a value of one, crack is initiated and the fracture energy is released to create two traction free surfaces. 


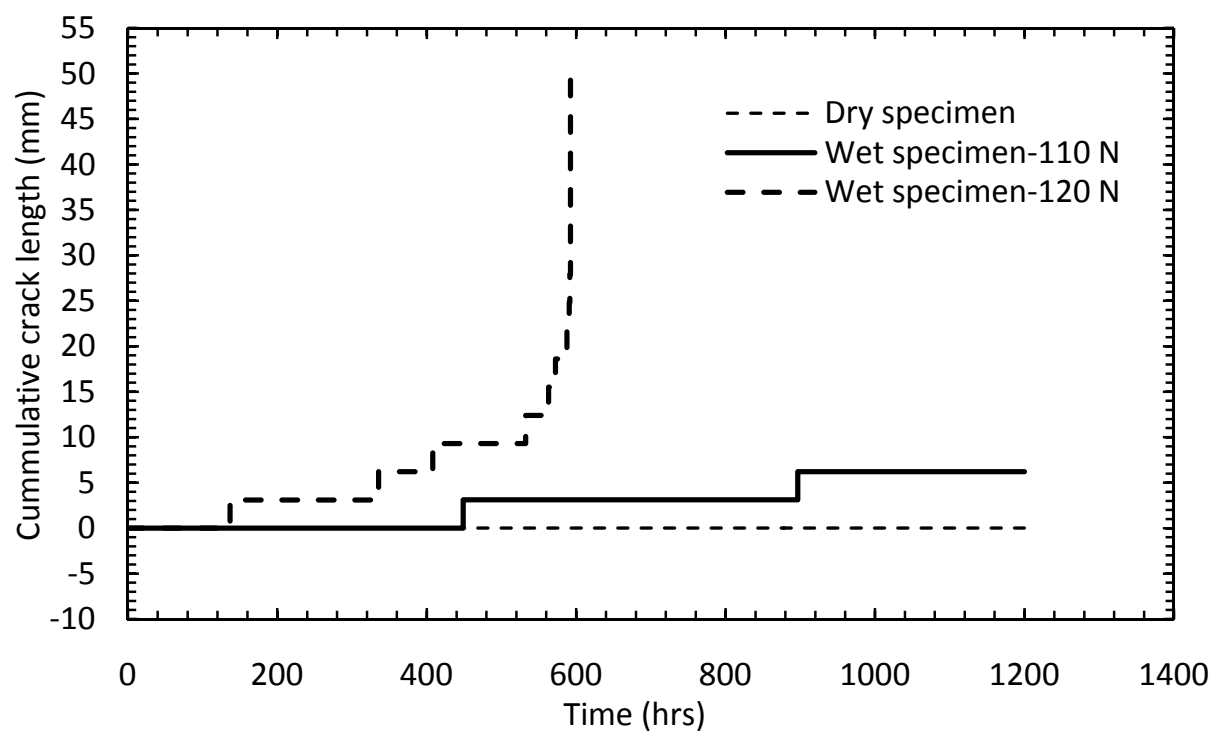

Fig. 4.4. Cumulative crack length vs. time for a DCB in mode I under creep loading with the crack at the skin-core interface

The analysis is performed for two different constant load levels of 110 and 120 $\mathrm{N}$, which correspond to $46 \%$ and $50 \%$ of the failure load of the dry specimen respectively. Fig 4.4 shows the graph of cumulative crack length versus time for these applied loads of $110 \mathrm{~N}$ and $120 \mathrm{~N}$ and the moisture concentration of $1 \mathrm{gm} / \mathrm{mm}^{3}$ at the top surface with an assumed value of critical displacement as $0.34 \mathrm{~mm}$ and the predetermined failure paths between the skin and adhesive and between core and adhesive. With the increase in the time the moisture diffusion progresses and the material properties degrade further. As a result the crack propagates ahead at the interface as the time increases. From the FE simulation it is observed that for the same sandwich beam in a dry condition (without moisture concentration), with the same value of the applied load the crack does not propagate till time $\mathrm{t}=1200$ hours. This indicates 
the detrimental effect of moisture concentration on crack propagation. Fig 4.5 shows the test results for the predetermined failure path within the adhesive with the same geometry, boundary and loading conditions and failure criterion.

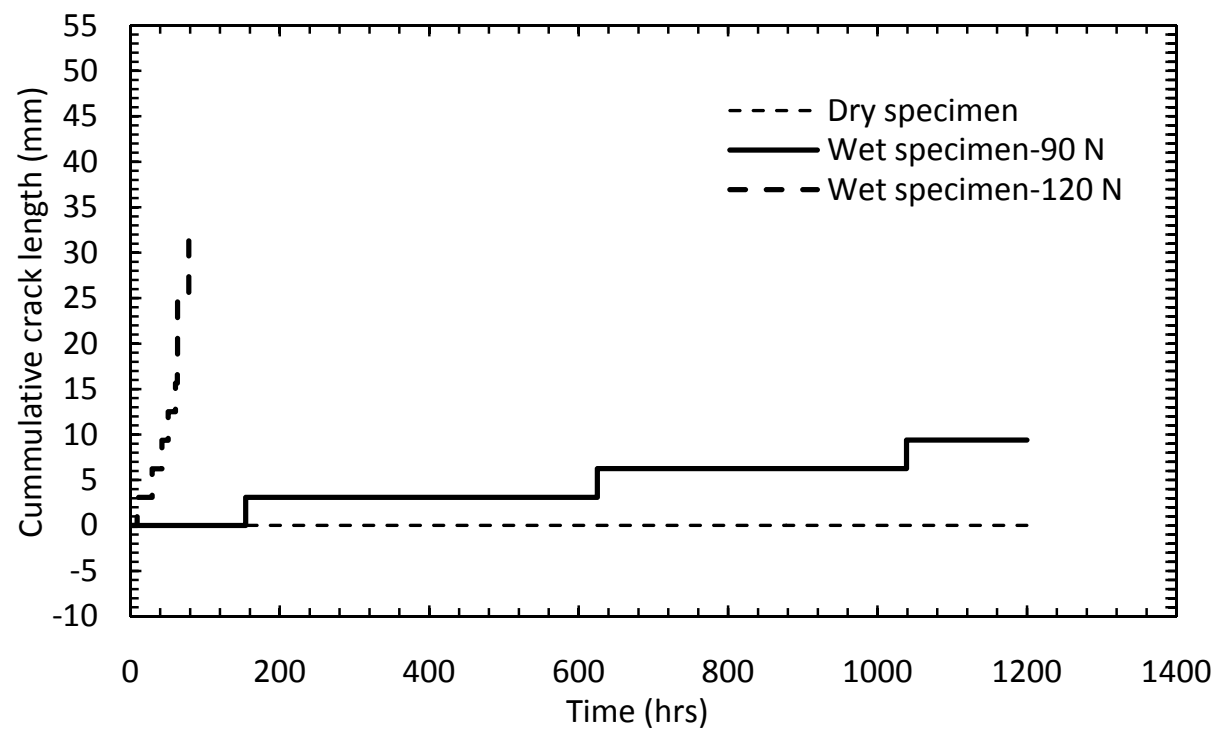

Fig. 4.5. Cumulative crack length vs. time for a DCB in mode I under creep loading with the crack within the glue

A similar analysis is performed using the TSD test for a $10^{\circ}$ tilt angle $(\theta)$ for the same geometry. Fig 4.6 shows the graph of cumulative crack length versus time for the applied loads of $120 \mathrm{~N}$ and $130 \mathrm{~N}$ and the moisture concentration of $1 \mathrm{gm} / \mathrm{mm}^{3}$ at the top surface with value of critical displacement as $0.34 \mathrm{~mm}$ [33] and the predetermined failure path between the skin and adhesive and between core and adhesive. 


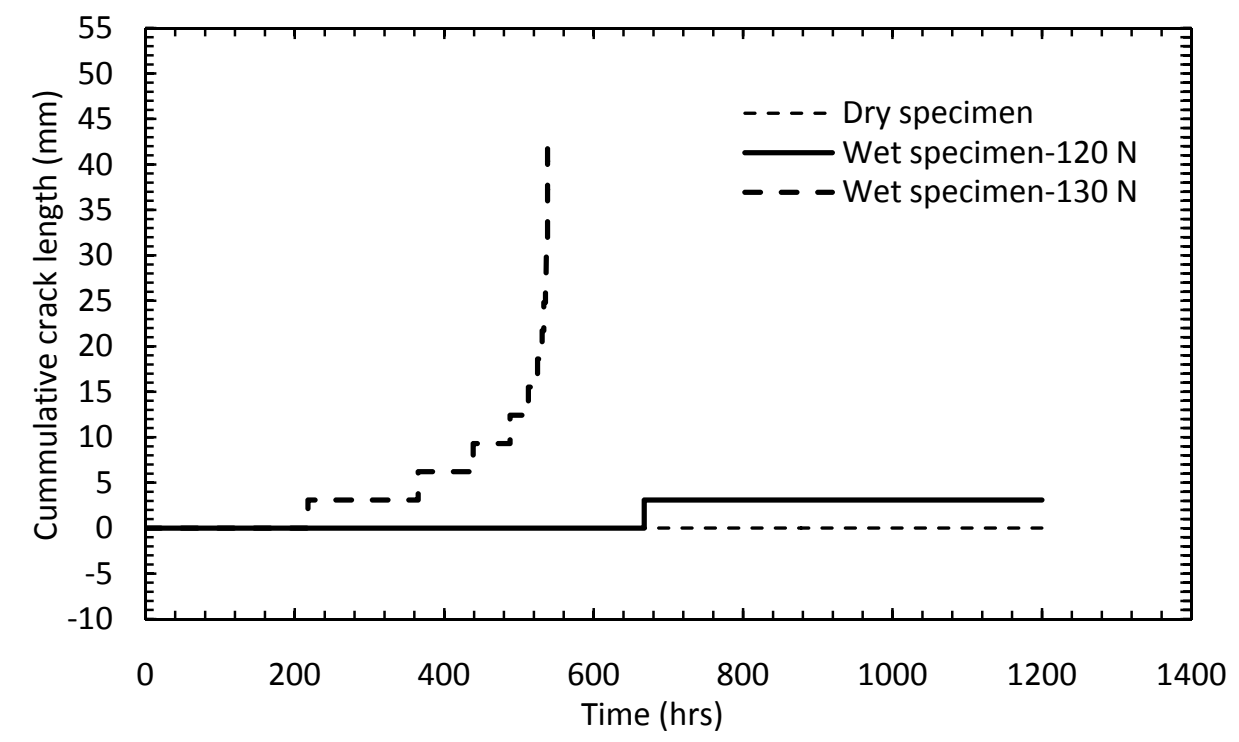

Fig. 4.6. Cumulative crack length vs. time for a TSD beam in mode I under creep loading

Another failure criterion that can govern the crack propagation at the interface between the skin and the core is the critical stress criteria. Depending upon the material properties, the bond strength between the skin and the adhesive and the adhesive and the core, the uniformity with which the adhesive is applied at the skin-core interface etc. the failure can either be governed by the COD or by the critical stress criterion. In case of the availability of the material data, the crack propagation using the COD or the critical stress criterion can be predicted using the FE analysis. This section of the study highlights the effects of moisture on the crack propagation using critical stress criterion. The critical stress criterion is defined as [40]:

$$
f=\sqrt{\left(\frac{\sigma}{\sigma_{c r}}\right)^{2}+\left(\frac{\sigma}{\sigma_{c r}}\right)^{2}}
$$


Where $\sigma_{\mathrm{cr}}$ and $\tau_{\mathrm{cr}}$ are the normal and shear critical stress, respectively.

Fig 4.7 shows the graph of cumulative crack length versus time for the applied loads of $110 \mathrm{~N}$ and $120 \mathrm{~N}$ and the moisture concentration of $1 \mathrm{gm} / \mathrm{mm}^{3}$ at the top surface with assumed values of normal and shear critical stress as $5 \mathrm{MPa}$ and $2 \mathrm{MPa}$ respectively and the predetermined failure paths between the skin and adhesive and between core and adhesive. The critical stresses are determined from the stresses fields near the crack tip developed in the dry specimen.

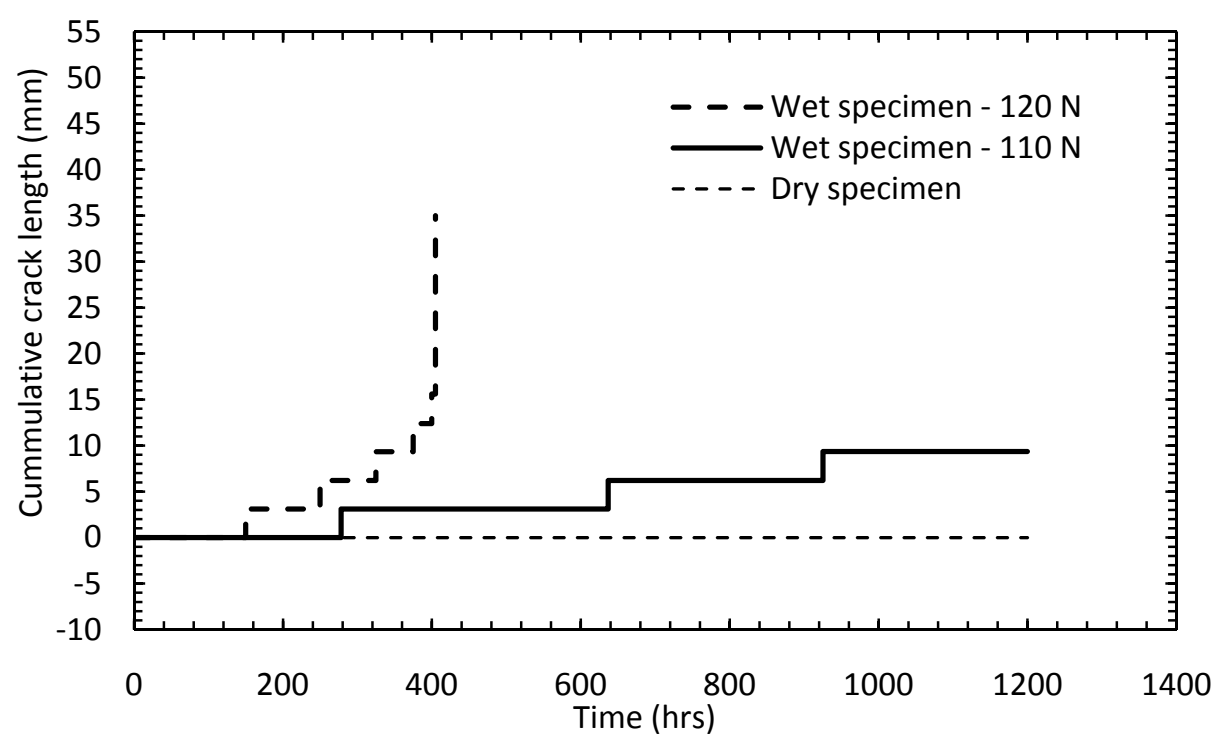

Fig. 4.7. Cumulative crack length vs. time for a DCB in mode I under creep loading with the crack at the skin-core interface using the critical failure stress criterion

Fig 4.8 shows the Von Mises stress plots at the crack tip at the times just before and after the crack opens. As expected high stresses are observed at the crack tip before 
the crack opens. Eventually as the crack opens two traction free surfaces are generated which results into stress free surfaces as shown in the figure.

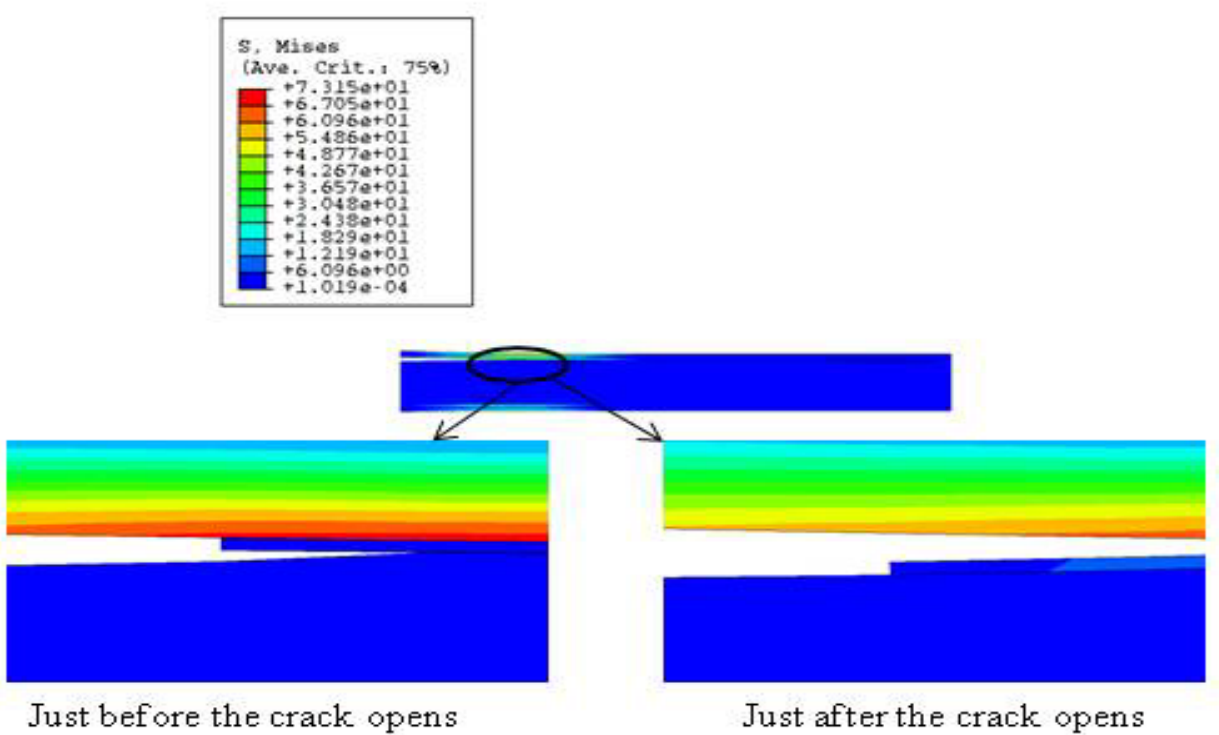

Fig. 4.8. Von Mises stress plot at the crack tip

From this study it is observed that the depending upon the failure criterion (COD or the critical failure stress) governing the delamination at the interface, the cumulative crack length and the time of crack initiation are affected. In practice it is essential to know whether the delamination is governed by the COD criterion or critical failure stress criterion or a combination of both or some other criterion. This can depend upon the density of the core material, the strength of the glue and the uniformity with which the glue is applied at the skin-core interface. If it is possible to know the failure criterion 
from the experimental studies, FE method can then be used to predict the delamination at the interface.

\subsection{HYGROSCOPIC SWELLING IN SANDWICH PANELS}

Another major application of the sandwich composites is in the form of panels. The sandwich panels are used as floors in the airplanes, cabinet walls and several other naval structures. The epoxy resin in the carbon/epoxy laminate (skin of the sandwich composite) has a tendency to absorb moisture when exposed to moist environment. In addition to this, the polymeric cores also absorb moisture when exposed to moist environment. As a result of this, it is quite possible that large expansional strains (swelling) can be induced in the sandwich composite as a whole, resulting into significant stresses. Therefore, it is of prime interest to study the hygroscopic swelling and induced stresses in the sandwich panels when exposed to moist environment.

Abot et al. [41] experimentally analyzed the hygroscopic behavior of a woven fabric carbon-epoxy composite and its effect on the viscoelastic properties and glass transition temperature. The tests were conducted at full immersion in water and at a specific temperature condition. It was observed that the absorption in through-thethickness direction was determined to be lower than in the in-plane directions. Also the coefficients of moisture expansion were found to be similar in the warp and the fill directions but much lower in the through-the-thickness direction. It was also concluded that the viscoelastic properties were not affected during the process but the plasticization was very pronounced. Bouadi and Sun [11] analyzed the effect of humidity on the stress field of laminated composites induced due to the hygroscopic strains. A three- 
dimensional finite element approach was used to analyze the problem. It was observed that some of the stress components induced by the hygroscopic change are significant to cause failure of the laminate. Cairns and Adams [42] presented experimental techniques for determining the moisture and thermal expansion coefficients of polymers and polymer-matrix composite materials. Experiments were conducted on Hercules 3501-6 neat epoxy resin, Hercules AS/3501-6 graphite/epoxy unidirectional composite. The results obtained from the experiments were found to be in good agreement with the numerical predictions. The results obtained could serve as a base from which designers may account for moisture-induced stresses and strains in composites. Xiaoming and Weitsman [43] studied the effects of sea-water on foam cored sandwich structures under long-term exposure with the focus on the sea-water induced damage in foam materials, weight gains and expansional strains. Two closed-cell polymeric foam materials (H100 and H200 PVC), and their polymeric composite facing sandwich specimens, immersed in the sea water showed a substantial amount of swelling strains.

In this study, the effect of moisture induced swelling on the behavior of the sandwich panel is analyzed. A sandwich panel 1200 X 1000 X $50 \mathrm{~mm}$ (as per the panels manufactured by Beijing Baodu Steel Structure Project Limited Company) is subjected to moisture concentration on the top surface of the panel. The skin is made up of orthotropic carbon/epoxy laminate (1 $\mathrm{mm}$ thick) and the core is isotropic linear viscoelastic polyurethane (48 $\mathrm{mm}$ thick). The instantaneous part $\left(\mathrm{E}_{0}\right)$ of the polyurethane foam core is assumed to degrade linearly with the moisture concentration as per the Eq. (3.13). FE approach is used to simulate this problem of moisture induced 
swelling. The geometry is modeled using continuum 3D diffusivity elements (DC3D8) to account for the moisture diffusion problem and (C3D8) elements to model the expansional strains. The analysis is performed upto 1200 hours (greater than the steady state time of 466 hours required for the moisture diffusion process). As discussed by Springer [44] the moisture concentration applied at the surface of the material, denoted by $c_{m}$ is related to the relative humidity by the empirical relationship:

$$
c_{m}=\rho_{d} a H^{b}
$$

Here $\rho_{\mathrm{d}}$ is the density of the dry material, $\mathrm{H}$ is the relative humidity and $\mathrm{a}$ and $\mathrm{b}$ are empirical constants which depend on the material. For the carbon-epoxy facing exposed to $100 \%$ relative humidity $(\mathrm{RH})$ the values of these empirical constants as given by Loos et al. [45] are $a=0.018$ and $b=1$. For these values of $a, b$ and $R H$ the applied moisture concentration at the surface of the carbon-epoxy facing is $2.916 \mathrm{e}-5 \mathrm{gm} / \mathrm{mm}^{3}$. For the applied moisture concentration of $2.916 \mathrm{e}-5 \mathrm{gm} / \mathrm{mm}^{3}$ at the top surface of the panel and assumed moisture diffusivity of the skin and the core as $1.06 \mathrm{E}-3 \mathrm{~mm}^{2} / \mathrm{sec}$ and $1.06 \mathrm{E}-2 \mathrm{~mm}^{2} / \mathrm{sec}$ respectively, the moisture diffusion process reaches a steady state (fully saturated) at 432 hours. The known moisture diffusion profile is then used to couple with the expansional swelling. The coefficient of moisture expansion (CME) for the skin along the fiber direction $\left(\beta_{1}\right)$ and perpendicular to the fiber directions $\left(\beta_{2}, \beta_{3}\right)$ as per Cairns and Adams [42] are given in Table 4.3. Since, the value of CME for the polyurethane foam core is not available in the literature, an assumed value of 200 $\mathrm{mm}^{3} / \mathrm{gm}$ of that of a polymeric material used in the electronic packaging [46] has be 
used to perform the analysis. The panel is constrained as shown in the Fig 4.9. The hygroscopic strains induced in the panel are written as:

$\varepsilon_{i}=\beta_{i}\left(c-c_{r e f}\right)=\beta_{i} \Delta c$

Here $\beta_{i}$ is the coefficient of moisture expansion and $c_{r e f}$ is the moisture concentration in reference state (the dry state).

Table 4.3. The coefficient of moisture expansion of skin and core material

\begin{tabular}{|c|c|c|}
\hline Material & $\begin{array}{c}\text { Coefficient of } \\
\text { Moisture expansion } \\
\left(\mathrm{mm}^{3} / \mathrm{gm}\right)\end{array}$ & Values \\
\hline Core: Polyurethane & $\beta$ & 200 \\
\hline $\begin{array}{c}\text { Skin: Carbon Epoxy } \\
\text { Composite(AS4/3501-6) }\end{array}$ & $\beta_{1}$ & 12.34 \\
& $\beta_{2}$ & 240 \\
& $\beta_{3}$ & 240 \\
\hline
\end{tabular}




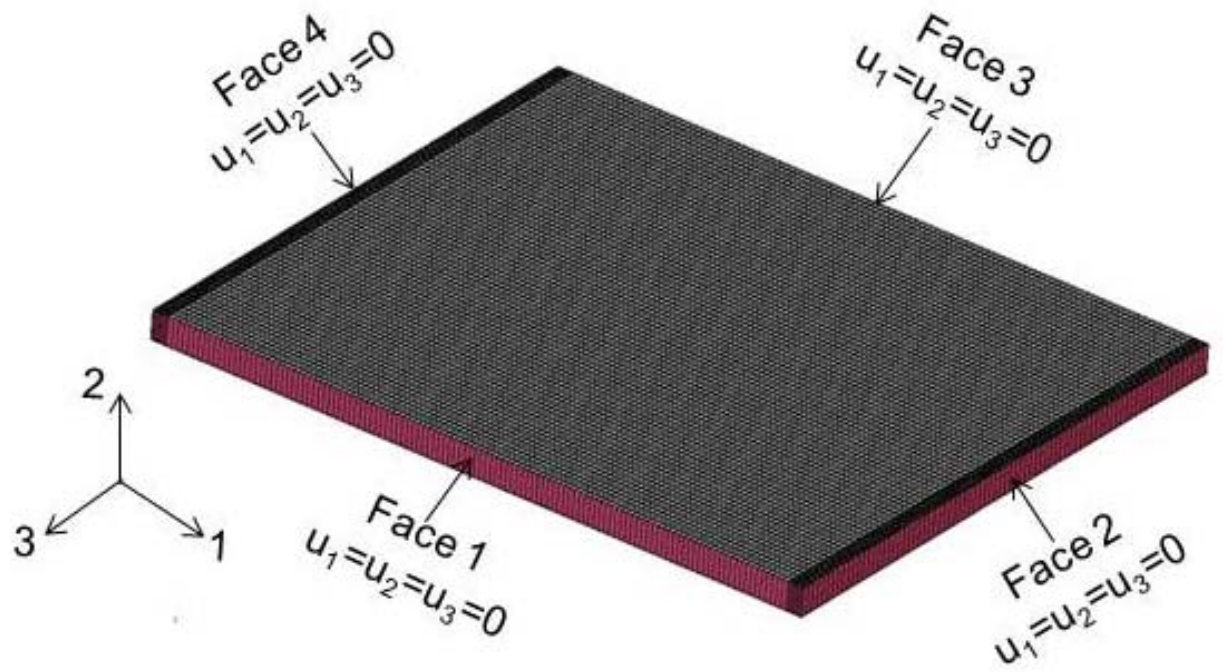

Fig. 4.9. Finite Element mesh and boundary conditions of the sandwich panel

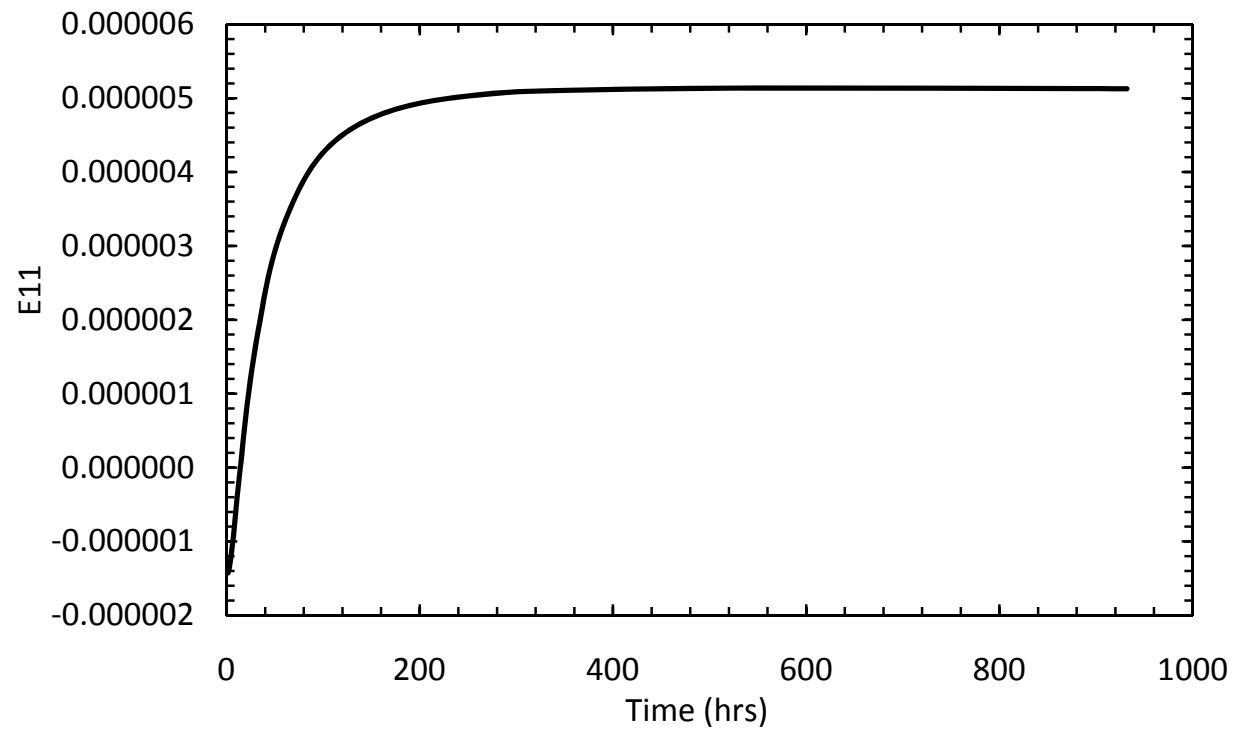

Fig. 4.10. Hygroscopic strain in the 1-direction (along the fiber) versus time 


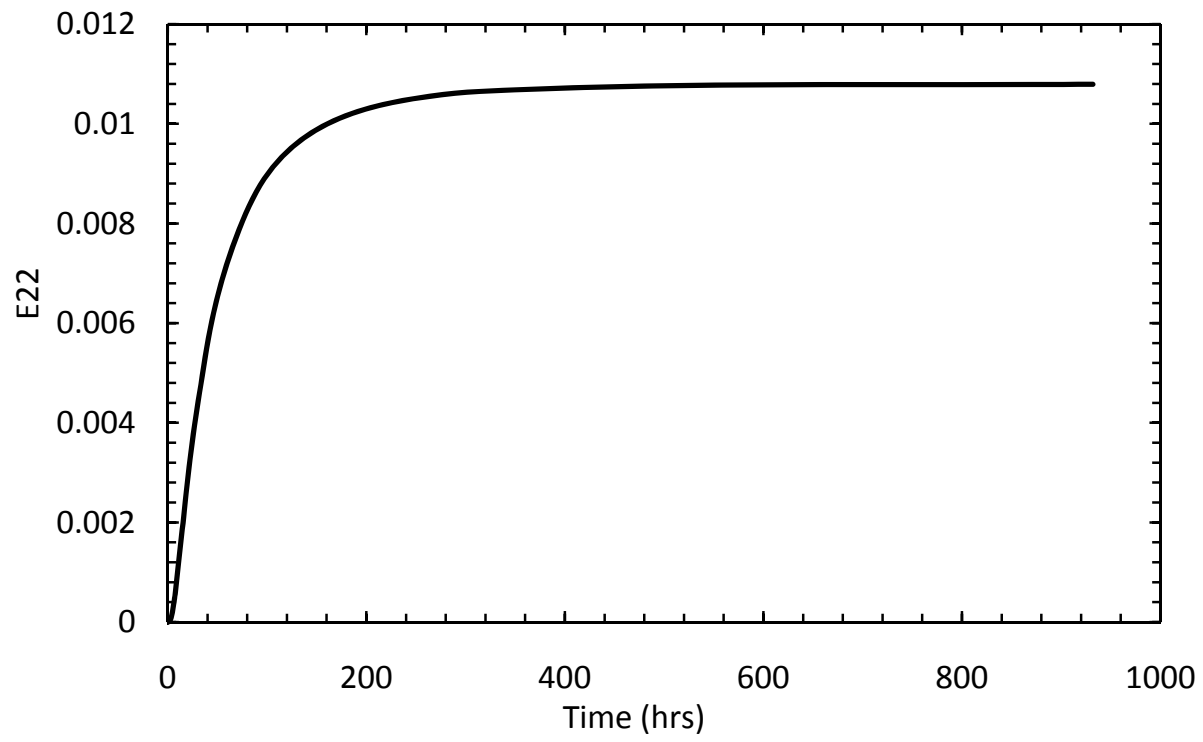

Fig. 4.11. Hygroscopic strain in the 2-direction (through the thickness) versus time

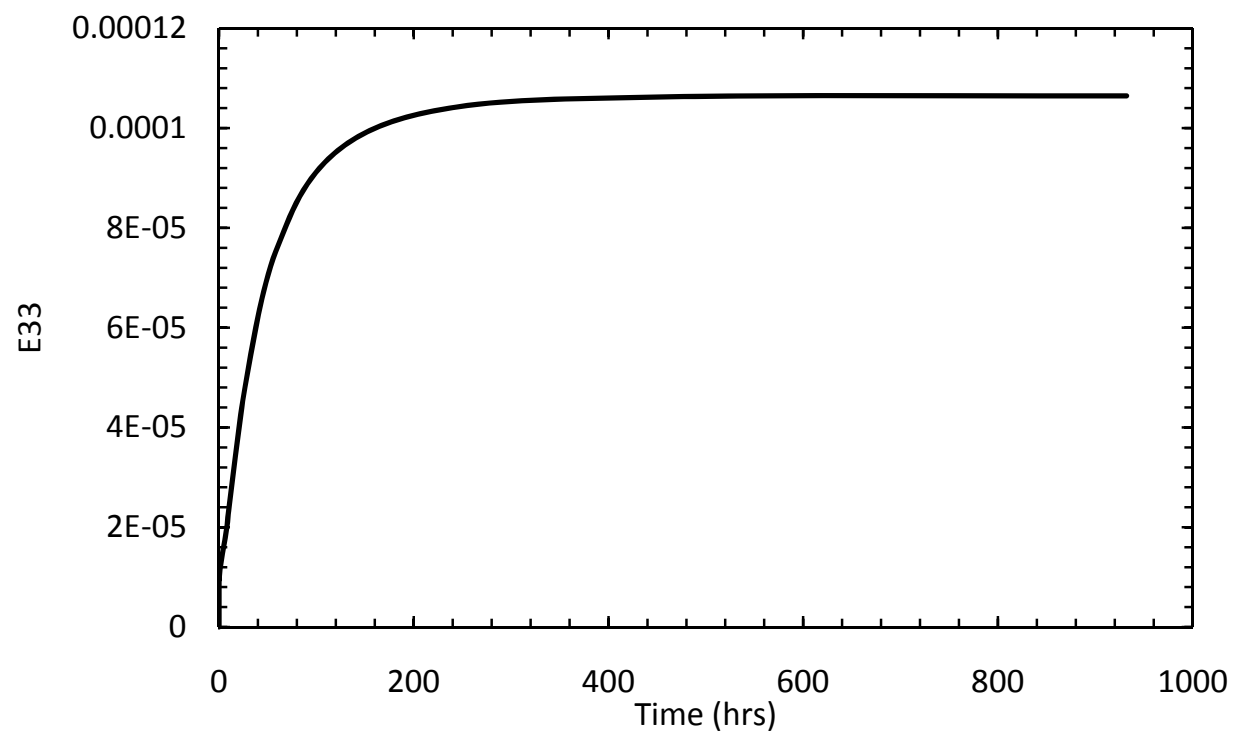

Fig. 4.12. Hygroscopic strain in the 3-direction (transverse) versus time 
The Fig 4.10 to 4.12 shows the hygroscopic strains induced in all the three directions due to the moisture absorption at the center on the surface of the bottom facing. The results are plotted for up to $\mathrm{t}=950$ hours. From the graphs it can be observed that significant changes in the strains are observed up to around 500 hours as the steady state is reached at 466 hours. For all the times greater than 500 hours the strains remain almost constant. As expected the hygroscopic strain in the fiber direction is quite less as compared to the other two directions. Whereas the strain in transverse to the fiber direction is quite significant. Maximum strain is observed in through the thickness directions as expected. These strains can be used in designing naval structures which are exposed to sever moisture concentrations as the hygroscopic strains lead to significant amount of stresses which can lead to the failure of the structure. Fig 4.13 shows von Mises stress plot for the sandwich panel at 1200 hours. Stresses of around 80 $\mathrm{MPa}$ in the fiber direction (1-direction), around $70 \mathrm{MPa}$ in the through the thickness direction (3-direction) are developed in the center region of the skin at 1200 hours. As expected negligible stresses are developed in the transverse to the fiber direction (2direction) since the body is free to expand in the 2-direction except for constrained boundaries. Fig 4.14 show the von Mises stress plots for the skin (top and bottom). Negligible stresses are developed in the core as shown in the Fig 4.15. 


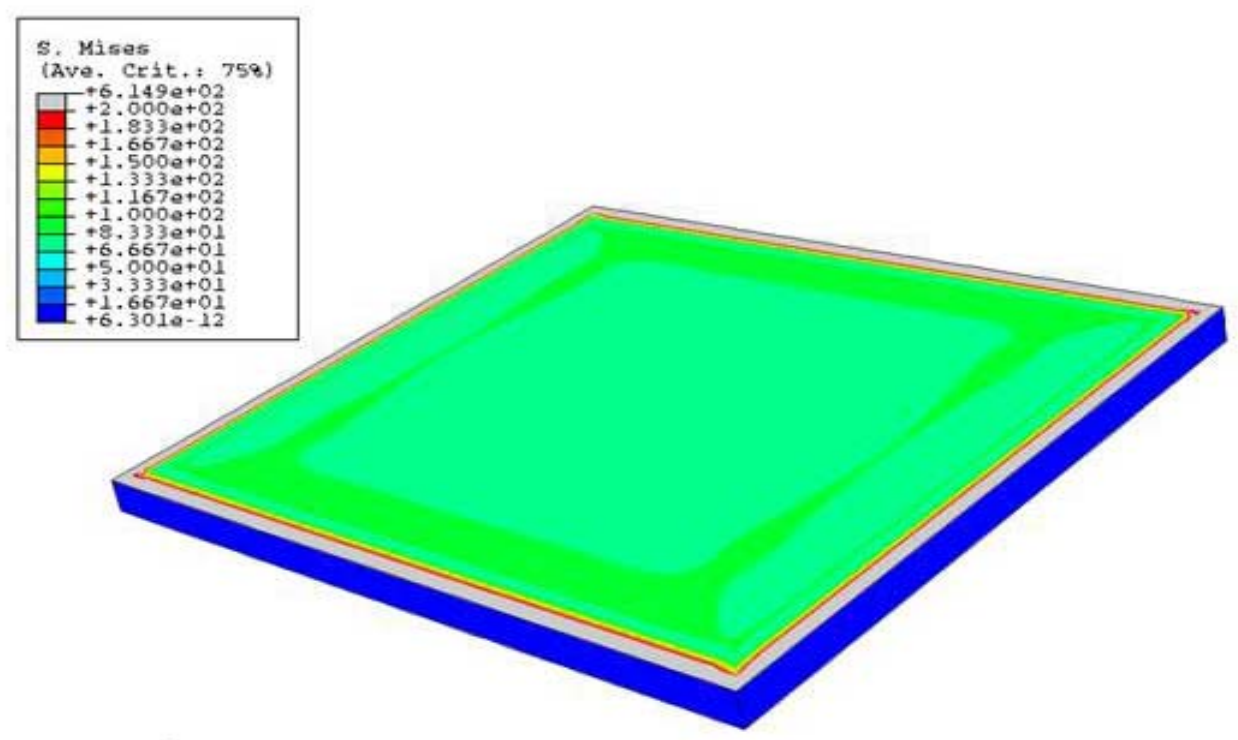

Fig. 4.13. Von Mises stress plot for the sandwich panel

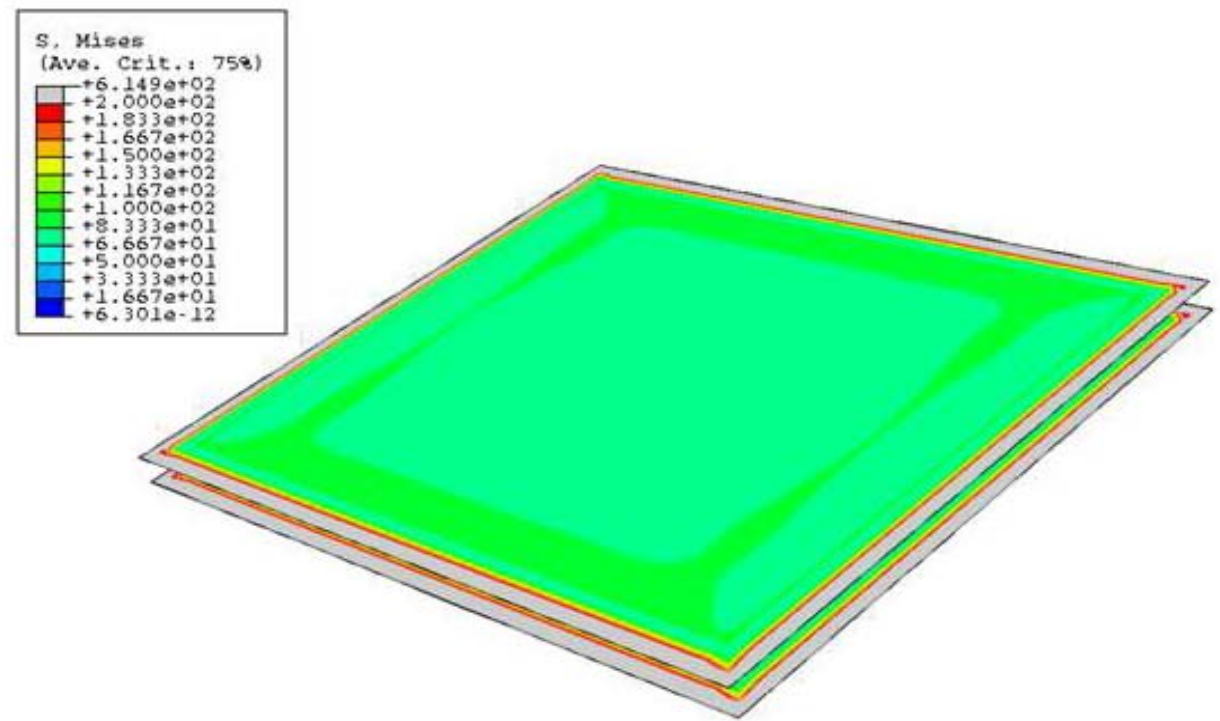

Fig. 4.14. Von Mises stress plot for the skin 


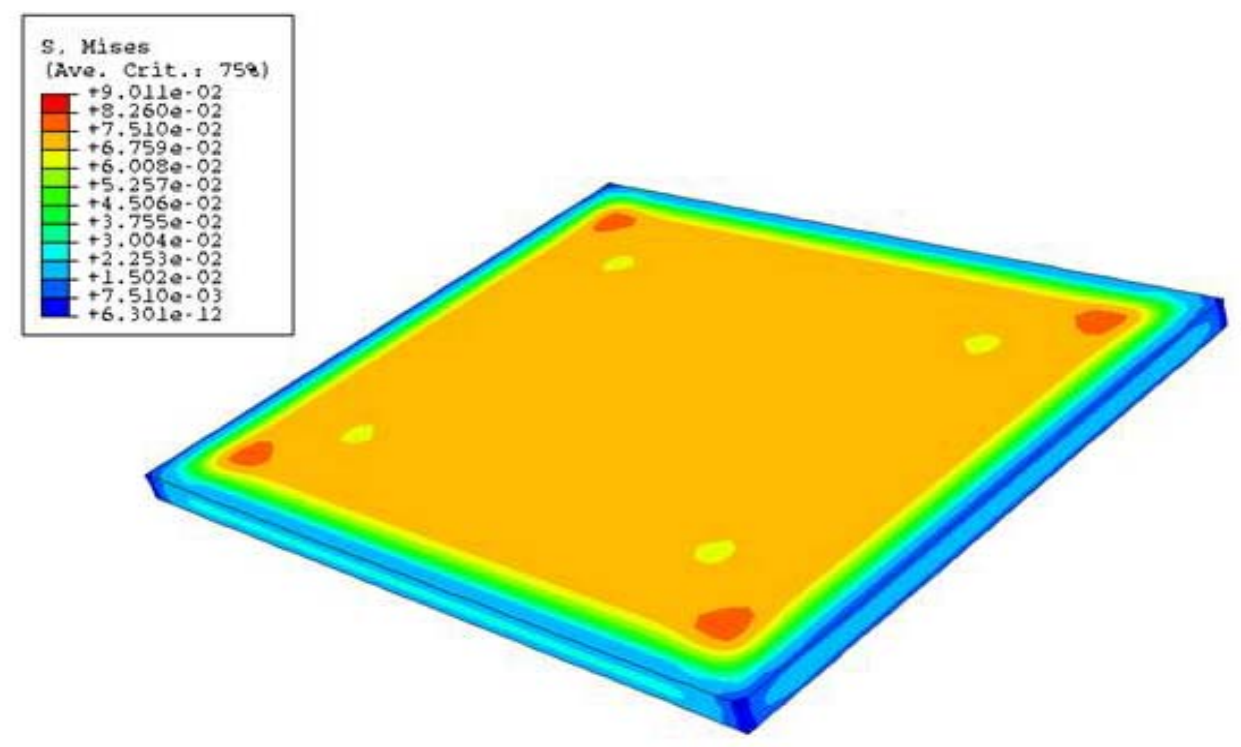

Fig. 4.15. Von Mises stress plot for the core 


\section{CHAPTER V}

\section{CONCLUSIONS AND FURTHER RESEARCH}

\subsection{CONCLUSIONS}

Effect of moisture diffusion on the deformation of viscoelastic sandwich composites, which are composed of orthotropic fiber-reinforced laminated skins and viscoelastic polymeric foam core, is analyzed. It is assumed that the elastic and timedependent (transient) moduli at any particular location in the foam core depend on the moisture concentration at that location. Sequentially coupled analyses of moisture diffusion and viscoelastic deformation are performed to predict overall performance of the studied sandwich systems. The analysis is performed in two sequential steps. In the first step the moisture concentration profile throughout the sandwich composite is obtained using the unsteady Fick's law of diffusion. In the next step, the time-dependent deformation is performed with the materials properties allowed to degrade with the moisture concentration, so as to highlight the impact of moisture on the field variables. Since the viscoelastic materials exhibit a history dependent response, it becomes very essential to incorporate the effect of stress, strain histories. Because, the material moduli are dependent on the moisture concentration which is directly affects in the field variables there arises a need to perform a sequential analysis so as to capture the history dependent field variables (stress and strain). Time and moisture dependent constitutive model is used for polymer foam core. A time-integration algorithm is developed to link this constitutive model to finite element (FE) analyses framework. 2D plane strain and 3D continuum elements are used to analyze the overall time-dependent responses of the 
sandwich composites subject to moisture diffusion. The results obtained from the FE code are verified with the analytical models and the available experimental data. Since FE code is used to perform the analysis, it becomes essential to analyze the effect of various parameters such as the time increment, tolerance and the size of mesh on the overall response of the sandwich structure. A detailed convergence study is performed to obtain the values of theses parameters which ensures that the results obtained are within the required accuracy. Parametric studies on the effects of different diffusivity ratios of skin and core materials on stress, strain and displacement fields are analyzed. It is observed that for a higher diffusivity ratio the effect of moisture on the field variables is more pronounced in the initial times because the material properties degrade at a faster rate. Since viscoelastic materials are history dependent the effect of material properties degrading at different rates has a varied effect on the field variables. For all times greater than the steady state time (for the case with the least diffusivity ratio), the differences in the (corresponding) field variables with different diffusivity ratios is almost negligible. This is because the viscoelastic materials posses a property of fading memory due to which the effect of the previous histories at a longer time becomes negligible. Contributions of moisture dependent elastic and the time-dependent moduli to the overall stress, strain and displacement field for a specific case of the diffusivity ratio of 0.1 are studied. To accomplish this, two different cases were considered. In the first case only the instantaneous part was allowed to degrade with moisture whereas in the second both the instantaneous and the transient part were allowed to degrade with moisture. At the initial times the differences between the field variables in the two cases 
was negligible since the degradation of the transient modulus with moisture is not very significant. But with the increase in the time the moisture diffusion through the sandwich beam progresses and the creep compliance for the two cases is significantly different. This results into significant differences in the field variables. For all the times greater than the steady state time of the moisture diffusion, a nearly constant difference in the field variables is observed in the two cases as expected. The effect of moisture on the phenomenon such as delamination at the skin-core interface in the sandwich beam and hygroscopic swelling induced in the sandwich panels due to moisture absorption is also analyzed. The time-dependent delamination at the skin-core interface of the sandwich structure under coupled moisture and mechanical stimuli is analyzed, so as to highlight the impact of moisture concentration on the delamination at the interface. FM73M adhesive is used to glue the skin and the core of the sandwich composite. Time and moisture dependent constitutive model used for the polymeric foam core is also used to model the FM73M adhesive at the interface, with different moisture dependent parameters. Another application of the sandwich composites in the form of sandwich panels subjected to moisture loading is also analyzed. The hygroscopic swelling induced in a sandwich panel fixed in the four sides due to the moisture absorption in the skin and the core material is found to be significant.

\subsection{FURTHER RESEARCH}

The current study on the effect of moisture concentration in the sandwich composites is limited to linear viscoelastic polymeric foam core. The study can be extended to non-linear viscoelastic response of these polymeric foam cores. The stress 
and moisture dependent material properties can be incorporated to obtain the stress, strain and displacement fields. In the present study the coupled problem of moisture diffusion and viscoelastic deformation is analyzed where in the viscoelastic deformation at a particular instant time depends on the moisture concentration at that instant, but the moisture concentration at any time is obtained without the knowledge of stress or strain at that instant time. A fully coupled problem where in the stress or strain assisted moisture diffusion coupled with the deformation in the viscoelastic sandwich can be analyzed. As the sandwich composites can be exposed to severe temperature and moisture conditions, a combined effect of both moisture and temperature on the viscoelastic deformations in the sandwich composites can be analyzed. 


\section{REFERENCES}

[1]. Shen CH, Springer GS. Effect of Moisture and Temperature on the Tensile Strength of Composite Materials. Journal of Composite Materials 1977;11(1):2-16.

[2]. Springer GS, Sanders BA, Tung RW. Environmental Effects on Glass Fiber Reinforced Polyester and Vinylester Composites. Environmental Effects on Composite Materials 1981;1(1):126-144.

[3]. Antoon MK, Koening JL. Irreversible Effects of Moisture on the Epoxy Matrix in Glass-Reinforced Composites. Journal of Polymer Science 1981;19(2):197-212.

[4]. Burcham LJ, Vanlandingham MR, Eduljee RF, Gillespie JW. Moisture Effects on the behavior of Graphite/Polyimide Composites. Polymer Composites 1996;17(5):682-690.

[5]. Shen CH, Springer GS. Environmental Effects in the Elastic Moduli of Coposite Materials. Journal of Composite Materials 1977;11(3):250-264.

[6]. Snead JM. Moisture And Temperature Effects On The Instability Of Cylindrical Composite Panels. Journal of Aircraft 1983;20(9):777 -783.

[7]. Allred RE. The Effect of Temperature and Moisture Content on the Flexural Response of Kevlar/Epoxy Laminates: Part I. [0/90] Filament Orientation. Journal of Composite Materials 1981;15(2):100-116.

[8]. Gibson RF, Yau A, Mende EW, Osborn WE, Riegner DA. The Influence of Environmental Conditions on the Vibration Characteristics of Chopped- FiberReinforced Composite Materials. Journal of Reinforced Plastics and Composites, 1982;1(3):225-241.

[9]. Adams DF, Miller AK. Influence of Material Variability on the Predicted Environmental Behavior of Composite Materials. American Society of Mechanical Engineers 1977;77(9):7-7. 
[10]. Woldesenbet E, Gupta N, Vinson JR. Determination of the Moisture Effects on the Impact Properties of Composite materials. Journal of Materials Science 2002;37(13):2693-2698.

[11]. Bouadi H, Sun CT. Hygrothermal Effects on the Stress Field of Laminated Composites. Journal of Reinforced Plastics and Composites 1989;8:40-55.

[12]. Whitney JM, Ashton JE. Effect of Environment on the Elastic response of Layered Composite Plates. AIAA Journal 1971; 9(9):1708-1713.

[13]. Aiello MA, Leone M, Aniskevich AN, Starkova OA. Moisture Effects on Elastic and Viscoelastic Properties of CFRP Rebars and Vinylester Binder. Journal of Materials in Civil Engineering 2006;18(5):686-691.

[14]. Plushchik OA, Aniskevich AN. Effects of Temperature and Moisture on the Mechanical Properties of Polyester Resin in Tension. Mechanics of Composite Materials 2000; 36(3):395-407.

[15]. Zheng G, Kang Y, Sheng J, Qin O, Wang H, Fu D. Influence of Moisture Content and Time on the Mechanical Behavior of Polymer Material. Science in China Ser. E Engineering \& Materials Science 2004;47(5):595-607.

[16]. Harper BD, Rao JM, Kenner VH, Popelar CH. Hygrothermal Effects Upon Stress Relaxation in A Polyimide Film. Journal of Electronic Materials 1997;7(26):798804.

[17]. Flaggs DL, Crossman FW. Analysis of the Viscoelastic Response of Composite Laminates during Hygrothermal Exposure. Journal of Composite Materials 1981;15(1):21-40.

[18]. Botelho EC, Costa ML, Pardini LC, Rezende MC. Processing and Hygrothermal Effects on Viscoelastic Behavior of Glass Fiber/Epoxy Composites. Journal of Material Science 2005;40(14):3615-3623. 
[19]. Botelho EC, Costa ML, Rezende MC. Hygrothermal Effects on Damping Behavior of Metal/Glass Fiber/Epoxy Hybrid Composites. Materials Science and Engineering 2005;399(1-2):190-198

[20]. Fraga AN, Alvarez VA, Vazquez A, Osa O. Relationship Between Dynamic Mechanical Properties and Water Absorption of Unsaturated Polyester and Vinyl Ester Glass Fiber Composites. Journal of Composite Materials 2003;37(23):15531574.

[21]. Doxsee LE, Lee W, Springer GS, Chang SS. Temperature and Moisture Induced Deformations in Composite Sandwich Panels. Journal of Reinforced Plastics and Composites 1985;4(4):326-353.

[22]. Granville DM. Moisture Effects of Polymethacrylimide Foam and Honeycomb Core in Sandwich/Skin Structures. Annual Forum Proceedings - American Helicopter Society 1987;2(1):907-914.

[23]. Morganti F, Marchett M, Reibedli G. Effects of Moisture and Thermal Ageing on Structural Stability of Sandwich Panels. Acta Astronautica 1983;11(7-8):489-508.

[24]. Rajagopal KR, Wineman AS. Mechanical Response of Polymers:An Introduction. New York: Cambridge University Press; 2000.

[25]. Christensen RM. Theory of Viscoelasticity: An Introduction. New York, Academic Press: 1971.

[26]. Lee EH, Rogers TG. Solution of Viscoelastic Stress Analysis Problems Using Measured Creep or Relaxation Functions. Journal of Applied Mechanics 1963;30:127-133.

[27]. Haj-Ali RM, Muliana AH. Numerical Finite Element Formulation of the Schapery Non-linear Viscoelastic Material Model. International Journal for Numerical Mehtods in Engineering 2004;59:25-45. 
[28]. Taylor RL, Pister KS, Goudreau GL. Thermomechanical Analysis of Viscoelastic Solids. International Journal for Numerical Methods in Engineering 1970;2:45-59.

[29]. Weitsman Y. Stress Assisted Diffusion in Elastic and Viscoelastic Materials. Journal of Mechanics and Physics of Solids 1987;35(1):73-93.

[30]. Huang JS, Gibson LJ. Creep of Sandwich Beams with Polymer Foam Cores. Journal of Materials in Civil Engineering 1990;2(3):171-182.

[31]. Diab Inc., Diffusivity of Divinycell H-250, http:/fiberglasswarehouse.com/ tds/H_divinycell.pdf, 2002

[32]. Berggreen C, Simonsen BC, Borum KK. Experimental and Numerical Study of Interface Crack Propagation in Foam-cored Sandwich Beams. Journal of Composite Materials 2007;41(4):493-520.

[33]. Saha MC, Kabir E, Jeelain S. Study of Debond Fracture Toughness of Sandwich Composites with Nanophase Core. Materials Letters 2008;62:567-570.

[34]. Veazie DR, Robinson KR, Shivkumar K. Effects of the Marine Environment on the Interfacial Fracture Toughness of PVC Core Sandwich Composites. Composites Part B: Engineering 2004;35:461-466.

[35]. Scudamore RJ, Cantwell WJ. The Effect of Moisture and Loading Rate on the Interfacial Fracture Properties of Sandwich Structures. Polymer Composites 2002;23(3):406-417.

[36]. Jurf RA, Winson JR. Effect of Moisture on the Static and Viscoelastic Shear Properties of Epoxy Adhesives. Journal of Material Science 1985; 20:2979-2989.

[37]. Li X, Carlsson, LA. The Tilted Sandwich Debnd (TSD) Specimen for Face/Core Interface Fracture Characterization. Journal of Sandwich Structure Material 1999;1:60-75. 
[38]. Prasad S, Carlsson LA. Debondin and Crack Kinking in Foam Core Sandwich Beams II. Experimental Investigation. Engineering Fracture Mechanics. 1994;47:825-841.

[39]. Majumdar P, Srinivasagupta D, Mahfuz H, Joseph B, Thomas MM, Christensen S. Effect of Processing Conditions and Material Properties on the Debond Fracture Toughness of Foam-core Sandwich Composites: Experimental Optimization. Composites Part A: Applied Science and Manufacturing 2003;34:1097-1104.

[40]. ABAQUS, User's Manual, Version, 6.5, Hibbitt, Karlsson and Sorensen Inc., 2005.

[41]. Abot JL, Yasmin A, Daniel IM. Hygroscopic Behavior of Woven Fabric CarbonExpoxy Composites. Journal of Reinforced Plastics and Composites 2005;24(2):195-207.

[42]. Cairns DS, Adams DF. Moisture and Thermal Expansion Properties of Unidirectional Composite Materials and the Epoxy Matrix. Journal of Reinforced Plastics and Composites 1983;2:239-255.

[43]. Li X, Weitsman YJ. Sea-Water Effects on Foam-cored Composite Sandwich Layups. Composites Part B: Engineering 2004;35:451-459

[44]. Springer GS. Moisture Content of Composites Under Transient Conditions. Journal of Composite Materials 1977;11(1):107-123.

[45]. Loos AC, Springer GS. Moisture Absorption of Graphite Epoxy Composition Immersed in Liquid and Humid Air. Journal of Composite Materials 1979;13:131147

[46]. Wong EH, Rajoo R, Koh SW, Lim TB. The Mechanics and Impact of Hygroscopic Swelling of Polymeric Materials in Electronic Packaging. Transactions of the ASME 2002;124:122-126. 


\section{VITA}

Name: Nikhil P. Joshi

Address: Department of Mechanical Engineering, 3123 TAMU, College Station, TX 77843, USA

Email Address: nikhil_joshi@tamu.edu

Education: B.E, Production Engineering, Sardar Patel University, India, 2004

M.S, Mechanical Engineering, Texas A\&M University, College

Station, Texas, 2008 